\title{
The Strain/Stress Fields of a Subsurface Rectangular Dislocation Loop Parallel to the Surface of a Half Medium: Analytical Solution with Verification
}

\author{
Luo Li, Tariq A. Khraishi* , Abu Bakar Siddique \\ Mechanical Engineering Department, University of New Mexico, Albuquerque, NM, USA \\ Email: *khraishi@unm.edu
}

How to cite this paper: Li, L., Khraishi, T.A. and Siddique, A.B. (2021) The Strain/ Stress Fields of a Subsurface Rectangular Dislocation Loop Parallel to the Surface of a Half Medium: Analytical Solution with Verification. Journal of Applied Mathematics and Physics, 9, 146-175.

https://doi.org/10.4236/jamp.2021.91011

Received: December 2, 2020

Accepted: January 24, 2021

Published: January 27, 2021

Copyright $\odot 2021$ by author(s) and Scientific Research Publishing Inc. This work is licensed under the Creative Commons Attribution International License (CC BY 4.0).

http://creativecommons.org/licenses/by/4.0/

\begin{abstract}
The strain and stress fields of a rectangular dislocation loop in an isotropic solid that is a semi-infinite medium (half medium) are developed here for a Volterra-type dislocation. Specifically, the loop is parallel to the free surface of the solid. The elastic fields of the dislocation loop are developed by integrating the displacement equation of infinitesimals dislocation loops over a finite rectangular loop area below the free surface. The strains and stress then follow from the small strain tensor and Hooke's law for isotropic materials, respectively. In this paper, analytical verification and numerical verification for the elastic fields are both demonstrated. Equilibrium equations and strain compatibility equations are applied in the verification. Also, a comparison with a newly-developed numerical method for dislocations near a free surface is performed as well. The developed solution is a function of the loop depth beneath the surface and can be used as a fundamental solution to solve elasticity, plasticity or dislocation problems.
\end{abstract}

\section{Keywords}

Dislocation Loops, Free Surfaces, Volterra, Image-Stresses, Semi-Infinite Medium

\section{Introduction}

The problem of finding analytical solutions for the elastic fields of dislocations in different material types, material geometry and sizes has occupied researchers for tens of years. Early on, researchers focused their research on infinite isotropic materials or mediums. They also focused their work on infinitely-long dislo- 
cations (i.e. two-dimensional problems). For example, [1] [2] [3], amongst other textbooks, provided older known derivations for the displacement, strain and stress fields of screw and edge dislocations in an infinite medium assuming material isotropy. However, [2] has also provided two integral equations for finding the displacement field (the Burgers equation) and another stress fields of a closed dislocation loop (of any shape) in an infinite isotropic material.

Several researchers have studied different aspects of the dislocation loop problem using a variety of techniques. Initially, [4] and [5] investigated the prismatic circular loop (one whose Burgers vector is normal to its plane). The circular glide loop was initially investigated in [6] and given in [7]. The solution by Keller and Kröner was later corrected in [8] and [9]. In a more recent study of the displacement and stress fields of glide and prismatic circular dislocation loops, [10] [11] corrected some earlier work. The displacement field, including the solid angle term, of a rectangular dislocation loop of the Volterra type in an infinite medium was developed by [12].

A Somigliana ring dislocation having both prismatic and radial Burgers vector components was investigated by [13] [14]. A torsional dislocation loop of the Somigliana type was investigated by [15].

As for problems involving dislocations near a free surface, [16] derived the elastic fields of a dislocation meeting a surface in an angle for an arbitrary choice of Burgers vector. Baštecká [17] formulated the field stress due to a pure edge circular dislocation loop near a free surface with Burgers vector normal to that free surface. The effects of free surfaces on a circular loop were also studied by [18] and [19]. [20] [21] showed the field displacements due to an infinitesimal dislocation loop of arbitrary orientation and Burgers vector in a semi-infinite isotropic medium. The elastic field of a finite-sized closed dislocation loop can thus be obtained by means of area integration using the results for the infinitesimal loop. Jing et al. [22] used the last two references to find the displacement field of a rectangular dislocation loop parallel to a free surface. Maurissen and Capella [23] [24] derived the field stress correction terms of a dislocation segment parallel and perpendicular to a free surface in a semi-infinite elastic medium. Comninou and Dundurs [25] presented the formulations of the elastic field of an angular dislocation segment in isotropic half-space. For an anisotropic medium, [26] derived an integral form of field stress for the case of a dislocation terminating at the free surface of an anisotropic half-space. Gosling and Willis [27] expressed the stresses due to an arbitrary dislocation in a semi-infinite medium as a line integral along the dislocation.

In [1] [2], to treat the unphysical stress traction brought on the free surface by a screw dislocation line (parallel to the surface) whose fundamental solution is that of a screw dislocation in an infinite medium, an image screw dislocation with opposite Burgers vector is utilized. The image screw dislocation is a mathematical/un-crystal dislocation situated across from the surface at a distance equal to the crystal screw dislocation. The image solution does not satisfy the 
zero traction condition on the free surface when an edge dislocation parallel to the free surface is considered. In the case of an edge dislocation, [1] showed that extra term(s), called "surface correction terms" or stress surface correction terms, are needed to be added to the edge dislocation solution in an infinite medium plus the image edge dislocation solution (with opposite Burgers vector also treated as if it is in an infinite medium). With the addition of these three terms, the correct stress field is obtained as it ensures zero traction on the free surface.

In recent years, several papers utilized the "collocation point" numerical method to solve the problem of dislocation near a flat free surface. These collocation point methods enforce zero traction on a select number of surface points and not infinite number of them as in analytical methods. For example, [28] and [29] utilized an image dislocation plus a stress correction term. The stress correction term(s) were obtained by meshing the large surface with prismatic contiguous "mathematical" dislocation loops. Yan et al. [30] and [31] dropped the use of the image dislocations in the collocation point method by instead utilizing a mesh of generally-prismatic dislocation loops.

In this paper, the strain-stress field of the correction terms due to a rectangular Volterra dislocation loop parallel to a free surface are developed by building on the corrective displacement field solution (using [21] and [22]). Also, strainstress fields of the infinite medium term and the image term due to a rectangular dislocation loop are obtained by deriving the displacement field solution presented by [12]. Furthermore, analytical verification and numerical verification of the result will be presented.

Elastic field solutions for dislocation problems as presented herein, are beneficial for several reasons: 1) They serve as fundamental solutions, similar to a Green's function, for other elasticity, plasticity or dislocation problems (e.g. for disclination problems, fracture problems, or general eigenstrain problems [32] [33]), and 2) They serve as verification problems for numerical methods like the collocation-point method or different dislocation dynamic simulation codes.

\section{Elastic Fields of a Sub-Surface Rectangular Dislocation Loop}

First off, the problem configuration under consideration is given in Figure 1. The figure shows a free surface (labelled as such) of a half isotropic medium (i.e. semi-infinite medium) that is below the surface (i.e. lies in the positive $z$ or $x_{3}$ direction). Beneath the free surface is a rectangular dislocation loop (labelled as "Finite-sized crystal dislocation loop") that is parallel to free surface. This Volterra-type dislocation loop has a Burgers vector $\boldsymbol{b}$, which has three components $b_{x}, b_{y}$ and $b_{z}$ and has a dimension $2 a$ in the $x$-direction and a dimension $2 b$ in the $y$-direction. The line sense of the loop is shown with the arrow going around the loop. The loop is below the surface a distance $c$. The goal is to determine the strain and stress tensors or components for an arbitrary field material point $\mathrm{P}$. Note that in this paper, $x_{1}$ and $x$ are used interchangeably, so are $x_{2}$ and $y$, and so 


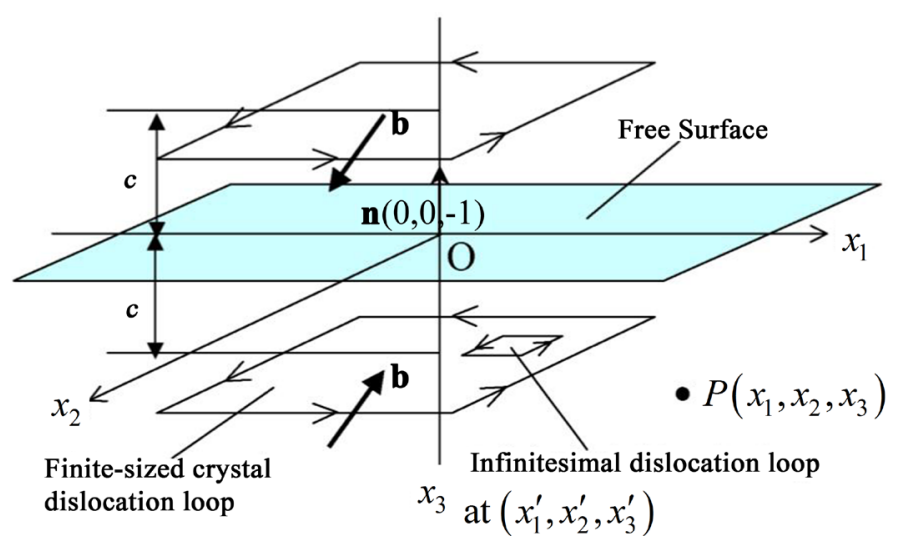

Figure 1. A finite-sized dislocation loop with an arbitrary Burgers vector, $\boldsymbol{b}$, below (by $c$ ) a free surface. Also shown an image dislocation loop with opposite Burgers vector above the surface. Lastly, an infinitesimal dislocation loop located at a point in the plane of the subsurface finite-sized loop is also shown.

are $x_{3}$ and $z$. Similarly for $x_{1}^{\prime}$ and $x^{\prime}$, and so forth.

To find the displacement, strain or stress fields ( $\vec{u}, \epsilon$ or, $\sigma$ respectively) due to the subsurface dislocation loop, such fields are the sum of three terms:

$$
\begin{aligned}
\vec{u} & =\vec{u}^{i n f}+\vec{u}^{i m a g e}+\vec{u}^{s} \\
\boldsymbol{\epsilon} & =\boldsymbol{\epsilon}^{i n f}+\boldsymbol{\epsilon}^{i m a g e}+\boldsymbol{\epsilon}^{s} \\
\boldsymbol{\sigma} & =\boldsymbol{\sigma}^{i n f}+\boldsymbol{\sigma}^{\text {image }}+\boldsymbol{\sigma}^{s}
\end{aligned}
$$

where the superscript "inf" refers to the field solution of a rectangular dislocation loop as if it was in an infinite medium and not in a half medium as shown in Figure 1, the superscript "image" refers to the field solution of an image rectangular dislocation loop which is also shown as the top loop in Figure 1 (also as if this image loop lied in an infinite medium), and finally the " $s$ " superscript refers to surface correction terms needed to ensure a zero traction condition on the free surface.

Let's focus first on the infinite term in the above equations. The Burgers equation [2] is an integral equation for the displacement field of a closed Volterra dislocation loop of any shape or curvature and lying in an infinite medium. It is composed of three integrals: the first of which is an area integral representing the solid angle of a rectangle, the second and third are line integrals summing the contributions of infinitesimal line lengths $\left(\mathrm{d} l^{\prime}\right)$ composing the loop along its line sense:

$$
\begin{aligned}
u_{m}(\boldsymbol{r})= & -\frac{1}{8 \pi} \int_{A} b_{m} \frac{\partial}{\partial x_{j}^{\prime}} \nabla^{\prime 2} R \mathrm{~d} A_{j}-\frac{1}{8 \pi} \oint_{c} b_{i} \in_{m i k} \nabla^{\prime 2} R \mathrm{~d} x_{k}^{\prime} \\
& -\frac{1}{8 \pi(1-v)} \oint_{c} b_{i} \in_{i j k} \frac{\partial^{2} R}{\partial x_{m}^{\prime} \partial x_{j}^{\prime}} \mathrm{d} x_{k}^{\prime}
\end{aligned}
$$

where $u_{m}$ is the $m^{\text {th }}$ component of the displacement vector $\vec{u}, b_{m}$ is the $m^{\text {th }}$ component of the displacement vector $\vec{b}=\boldsymbol{b}=\left(b_{x}, b_{y}, b_{z}\right), \in$ is the permutation symbol, $v$ is Poisson's ratio, $R=\sqrt{\left(x^{\prime}-x\right)^{2}+\left(y^{\prime}-y\right)^{2}+\left(z^{\prime}-z\right)^{2}}$ (see Figure 2) 
and $\nabla^{\prime 2} R=2 / R$.

The integration of the above equation for the displacement field of the rectangular dislocation loop shown in Figure 2 was done in [12]. It is not re-produced here. This displacement field represents the "inf" and "image" terms in Equation (1). To find the strain field associated with this rectangular dislocation loop in an infinite material, the tensorial small strain definition is invoked here:

$$
\epsilon_{i j}=\frac{1}{2}\left(\frac{\partial u_{i}}{\partial x_{j}}+\frac{\partial u_{j}}{\partial x_{i}}\right)
$$

This provides the "inf" and "image" terms in Equation (2). Finally, to find the stress field of this loop, one invokes Hooke's law for an isotropic material:

$$
\sigma_{i j}=\lambda \epsilon_{k k} \delta_{i j}+2 \mu \epsilon_{i j}
$$

where $\lambda=\frac{E v}{(1+v)(1-2 v)}, \quad \mu=\frac{E}{2(1+v)}$

Here, $\delta_{i j}$ is the $i j^{\text {th }}$ component of the Kronecker delta, $\mu$ is shear modulus, $\epsilon_{k k}$ is the dilatation or the volumetric strain, and $E$ is Young's modulus. Finding the stresses using Equation (6), will provide the "inf" and "image" terms in Equation (3). The strain and stress fields have been obtained here using the mathematical software Mathematica which has a very strong symbolic engine. However, they are not provided here for brevity for they will take several pages to list.

As for the displacement surface correction term in Equation (1), it can be obtained as follows:

$$
\vec{u}^{s}=\int_{A} \mathrm{~d} \vec{u}^{s}
$$

where $\mathrm{d} \vec{u}^{s}$ is the displacement vector at any field material point caused by an infinitesimal dislocation loop shown in Figure 1. By integrating the displacements caused by infinitesimal dislocation loops over a finite-sized area, one can obtain the displacement field associated with the sub-surface rectangular dislocation loop.

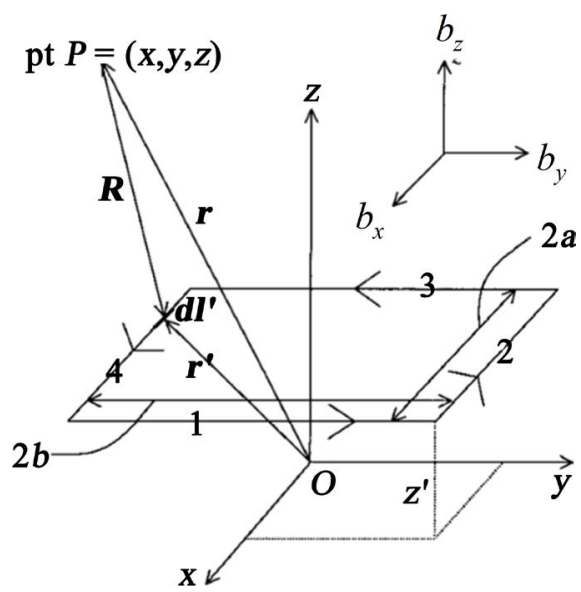

Figure 2. The geometry of a rectangular dislocation loop in infinite material. Here $\vec{r}^{\prime}=\boldsymbol{r}^{\prime}=\left(x^{\prime}, y^{\prime}, z^{\prime}\right)$. 
Bacon and Groves [21] provided a formula for the surface correction displacement term of a sub-surface infinitesimal dislocation loop, of area $\mathrm{d} S$ :

$$
\mathrm{d} u_{i}^{s}=-k x_{3}^{\prime}\left(1-2 \delta_{j 3}\right)\left[A_{i 3}\left(\frac{1}{R}\right)_{, i j}-\left(\frac{x_{3}}{R}\right)_{, i j 3}\right]
$$

where, $k=b_{j} \mathrm{~d} S / 4 \pi(1-v), \quad A_{i j}=2 v+4(1-v) \delta_{i j}, \mathrm{~d} S=\mathrm{d} x_{1}^{\prime} \mathrm{d} x_{2}^{\prime}$, $R^{2}=\left(x_{1}-x_{1}^{\prime}\right)^{2}+\left(x_{2}-x_{2}^{\prime}\right)^{2}+\left(x_{3}+x_{3}^{\prime}\right)^{2}$. The integration for the sub-surface rectangular dislocation loop was done by [22] and won't be re-produced here for brevity. Using such solution, the surface correction term for the strain field in Equation (2), can be obtained from Equation (5). Once this strain field is developed, the surface correction term for the stress field in Equation (3) can be obtained from Equation (6). The stress correction term is given here for the first time.

\section{Results and Discussion}

The surface correction terms for the strain and stress fields were obtained using the mathematical software Mathematica which has a very strong symbolic engine. Only the stress results are listed in the appendices (Appendix $\mathbf{A}$ for the $b_{x}$ component, Appendix B for the $b_{y}$ component, and Appendix C for the $b_{z}$ component). If one is interested in the surface corrections terms for strain, which are not listed here brevity, these could be obtained from the stresses in the appendices using:

$$
\epsilon_{i j}=\frac{1}{2 \mu}\left(\sigma_{i j}-\frac{\lambda \delta_{i j}}{2 G+3 \lambda} \sigma_{k k}\right)
$$

where $\sigma_{k k}$ is the first invariant of the stress tensor.

To verify the results, the authors embarked on several verifications: i-ensuring that the stress traction on the free surface is zero, ii-ensuring that the equilibrium equations are satisfied inside the half medium, iii-ensuring that the strain compatability equations are satisfied inside the half medium, and iv-comparing the analytical results with numerical results from the collocation-point method described above.

i) Stress Traction on the Free Surface

The stress traction $\vec{T}$ at the free surface is defined as:

$$
\vec{T}=\boldsymbol{\sigma} \vec{n}
$$

which should be $\overrightarrow{0}$ at the free surface. Here, $\sigma$ is given by Equation (3). However, the unit normal vector at the free surface is $\{0,0,-1\}$, see Figure 1 . This means from Equation (10) that $\sigma_{x z} \sigma_{y z}$ and $\sigma_{z z}$ should all be zero at the surface points. To check that these three stress components are zero on the surface, one could do one of two things. First, use Equation (3) and specify $z=0$ in it and see if it reduces to exactly 0 for each of the three stress components. Unfortunately, since the final results of Equation (3) are a few pages in length, Mathematica was not able to simplify these stress components at $z=0$ down to 0 
value even if one waited more than 24 hours for the simplification. Alternatively, one can consider arbitrary lines along the $x$ and $y$ directions on the free surface and see if these reduce to zero. They indeed all identically reduced to zero. In addition to this analytical verification, surface or carpet plots of the three stress components on the free surface were created. This is a numerical verification as all such stress values should be zero. The plots in Figure 3 show just that.

ii) Equations of Equilibrium

The partial differential equations of static equilibrium in a solid material are given in indicial notation by:

$$
\sigma_{i j, i}=\frac{\partial \sigma_{i j}}{\partial x_{i}}=0
$$

If one expands the last equation on the repeated indices then the resulting three explicit equations are:

$$
\begin{aligned}
& \frac{\partial \sigma_{x x}}{\partial x}+\frac{\partial \sigma_{x y}}{\partial y}+\frac{\partial \sigma_{x z}}{\partial z}=0 \\
& \frac{\partial \sigma_{y x}}{\partial x}+\frac{\partial \sigma_{y y}}{\partial y}+\frac{\partial \sigma_{y z}}{\partial z}=0 \\
& \frac{\partial \sigma_{z x}}{\partial x}+\frac{\partial \sigma_{z y}}{\partial y}+\frac{\partial \sigma_{z z}}{\partial z}=0
\end{aligned}
$$
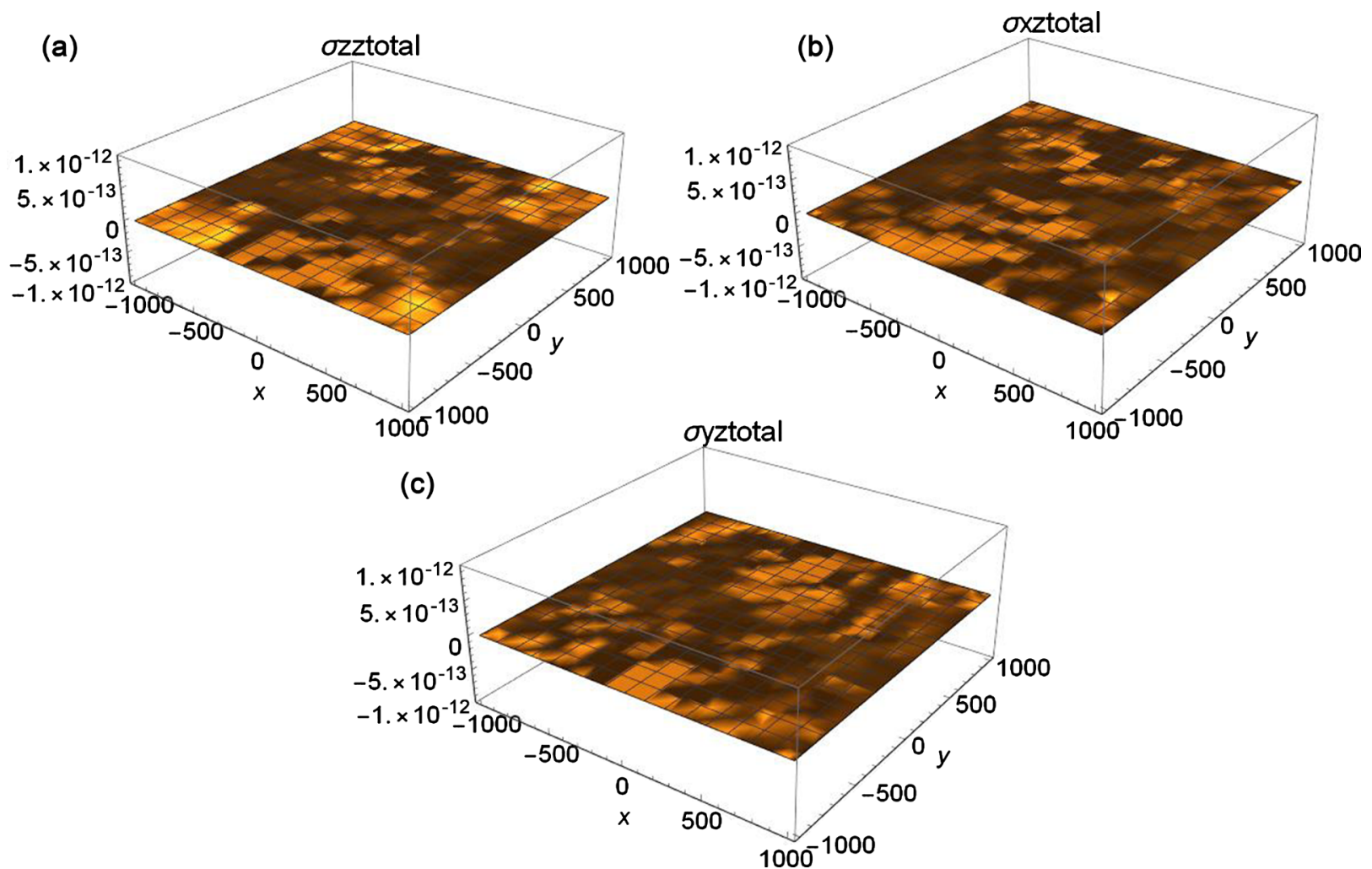

Figure 3. (a) Plot of $\sigma_{z z}$; (b) Plot of $\sigma_{x z}$; (c) Plot of $\sigma_{y z}$. For these plots, the following values were chosen: $a=b=100 b_{z}$, $c=10 b_{z}, b_{x}=b_{y}=0, b_{z}=1, \quad v=0.3, \mu=100, z=0,-10 a \leq x \leq 10 a,-10 b \leq y \leq 10 b$. 
These equations should be satisfied at every material point of a solid in equilibrium. To verify the developed stress solution $\sigma$ given by Equation (3), one can see if Equations (12)-(14) are identically zero either using analytical or numerical methods. For the analytical method, the equations are so humungous that Mathematica is not able to simplify them to 0 . However, for any given line in space along the $x$-, $y$ - or $z$-directions, Mathematica identically simplifies the equilibrium equations to zero. Hence analytical verification of the equilibrium equations is possible. Alternatively, numerical verifications can also be made by plotting Equations (12)-(14) along any plane in the material to see if the equations give a zero result. Figure 4 shows such plotting for $b_{y} \neq 0$ right below the subsurface dislocation loop. The figure shows that the equilibrium equations are satisfied. Note that given the combination of Burgers vector components and equilibrium equations a total of nine plots are minimally generated. However, only three plots for one of the Burgers vector components are shown here for brevity.

iii) Strain Compatibility Equations

The equations of compatibility can be written in indicial notation as [34]:

$$
\epsilon_{i j, k l}-\epsilon_{j l, i k}-\epsilon_{i k, j l}+\epsilon_{k l, i j}=0
$$

This equation can be expanded over the repeated indices and written explicitly as six different/unique equations:

$$
\frac{\partial^{2} \epsilon_{x x}}{\partial y^{2}}+\frac{\partial^{2} \epsilon_{y y}}{\partial x^{2}}=2 \frac{\partial^{2} \epsilon_{x y}}{\partial x \partial y}
$$
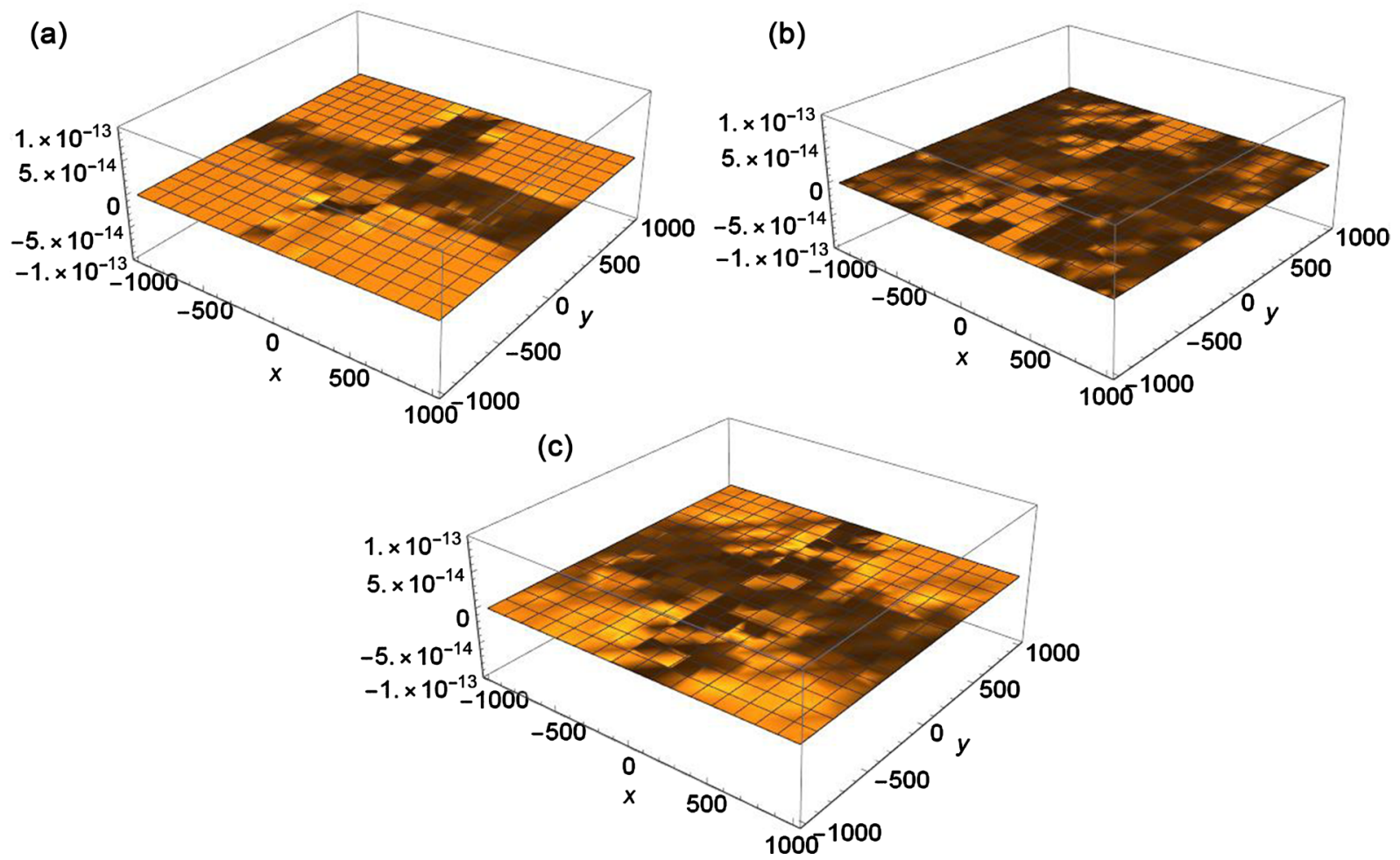

Figure 4. (a) Plot of equation (12); (b) Plot of equation (13); (c) Plot of equation (14). For these plots, the following values were chosen: $a=b=100 b_{y}, c=10 b_{y}, b_{x}=b_{z}=0, b_{y}=1, v=0.3, \mu=100, z=11 b_{y},-10 a \leq x \leq 10 a,-10 b \leq y \leq 10 b$. 


$$
\begin{gathered}
\frac{\partial^{2} \epsilon_{x x}}{\partial z^{2}}+\frac{\partial^{2} \epsilon_{z z}}{\partial x^{2}}=2 \frac{\partial^{2} \epsilon_{x z}}{\partial x \partial z} \\
\frac{\partial^{2} \epsilon_{z z}}{\partial y^{2}}+\frac{\partial^{2} \epsilon_{y y}}{\partial z^{2}}=2 \frac{\partial^{2} \epsilon_{z y}}{\partial z \partial y} \\
\frac{\partial^{2} \epsilon_{x x}}{\partial y \partial z}+\frac{\partial^{2} \epsilon_{y z}}{\partial x^{2}}=\frac{\partial^{2} \epsilon_{x z}}{\partial x \partial y}+\frac{\partial^{2} \epsilon_{x y}}{\partial x \partial z} \\
\frac{\partial^{2} \epsilon_{y y}}{\partial x \partial z}+\frac{\partial^{2} \epsilon_{x z}}{\partial y^{2}}=\frac{\partial^{2} \epsilon_{x y}}{\partial y \partial z}+\frac{\partial^{2} \epsilon_{y z}}{\partial x \partial y} \\
\frac{\partial^{2} \epsilon_{z z}}{\partial x \partial y}+\frac{\partial^{2} \epsilon_{x y}}{\partial z^{2}}=\frac{\partial^{2} \epsilon_{x z}}{\partial y \partial z}+\frac{\partial^{2} \epsilon_{y z}}{\partial x \partial z}
\end{gathered}
$$

These equations should be satisfied at every material point of a solid. To verify the developed stress solution $\epsilon$ given by Equation (2), one can see if Equations (16)-(21) are identically zero either using analytical or numerical methods. For the analytical method again, the equations are so large that Mathematica is not able to simplify them to 0 . However, for any given line in space along the $x$-, $y$ - or $z$-directions, Mathematica identically simplifies the equilibrium equations to zero. Hence analytical verification of the equilibrium equations is possible. Alternatively, numerical verifications can also be made by plotting Equations (16)-(21) along any plane in the material to see if the equations give a zero result. Figure 5 shows
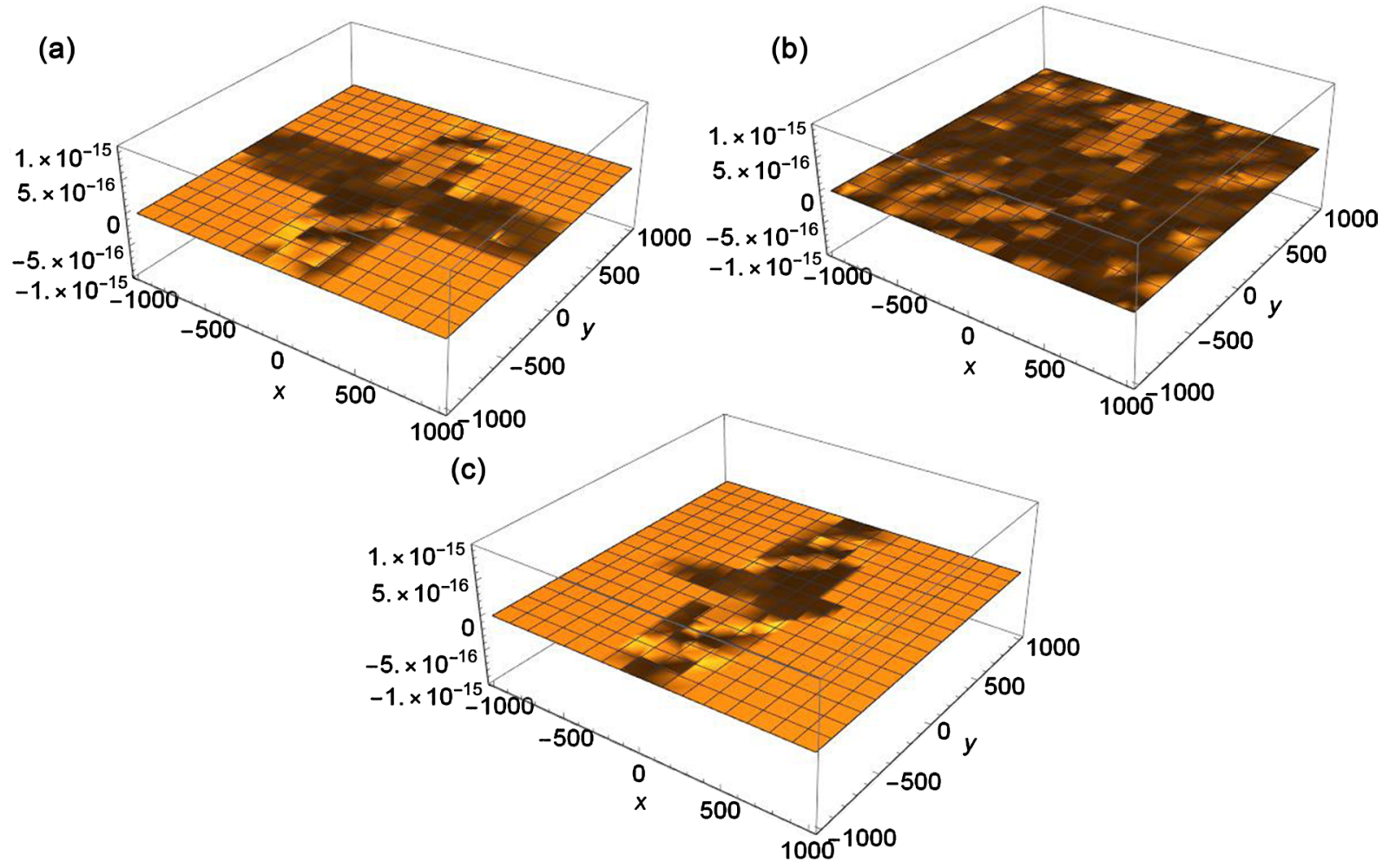

Figure 5. (a) Plot of Equation (16); (b) Plot of Equation (17); (c) Plot of Equation (18). For these plots, the following values were chosen: $a=b=100 b_{x}, c=10 b_{x}, b_{y}=b_{z}=0, b_{x}=1, \quad v=0.3, \mu=G=100, z=11 b_{x},-10 a \leq x \leq 10 a$, $-10 b \leq y \leq 10 b$. 
such plotting for $b_{x} \neq 0$ right below the sub-surface dislocation loop. The figure shows that the compatibility equations are satisfied. Note that given the combination of Burgers vector components and compatibility equations a total of eighteen plots are minimally generated. However, only three plots for one of the Burgers vector components are shown here for brevity.

iv) Comparison with the numerical "collocation point" method

As mentioned above, the "collocation point" method is a numerical method that can work in tandem with the infinite stress/strain terms to find the strain and stress fields in Equations (2) and (3). This numerical method works for any sub-surface dislocation geometry or inclination and not just horizontal dislocations or loops. To compare with this method, the following parameters were taken followed by stress components comparison between analytical and numerical solutions in Figures 6-15:

$$
\begin{gathered}
a=b=100, \quad c=400|\boldsymbol{b}|, \quad v=0.3, \quad z=200|\boldsymbol{b}|, \quad-10 a \leq x \leq 10 a, \\
-10 b \leq y \leq 10 b
\end{gathered}
$$

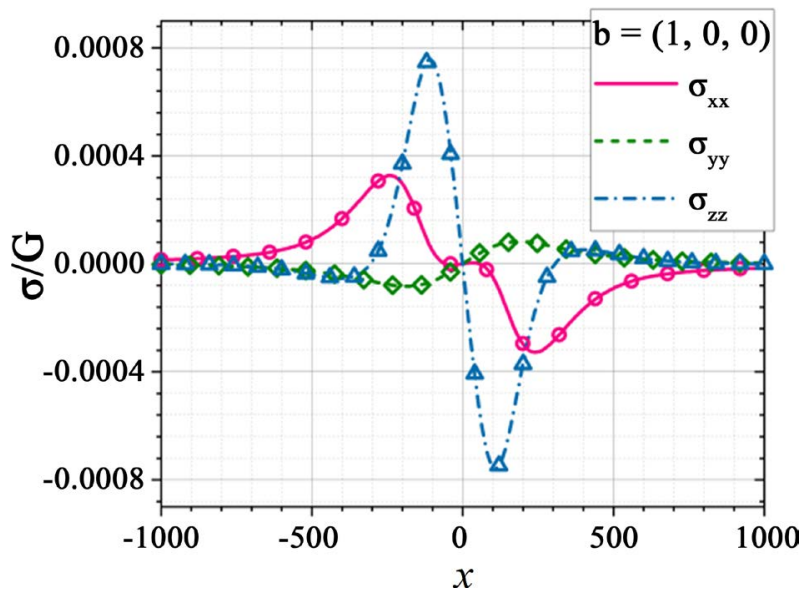

Figure 6. Comparison of analytical solutions (solid and dashed lines) to the results of the numerical "collocation point" method (symbols) along $x$-direction for non-zero $b_{x}$.

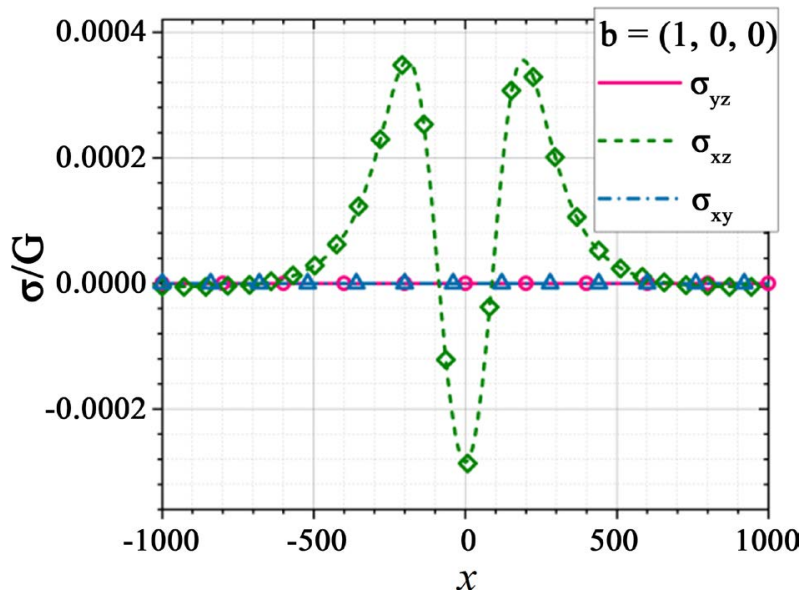

Figure 7. Comparison of analytical solutions (solid and dashed lines) to the results of the numerical "collocation point" method (symbols) along $x$-direction for non-zero $b_{x}$. 


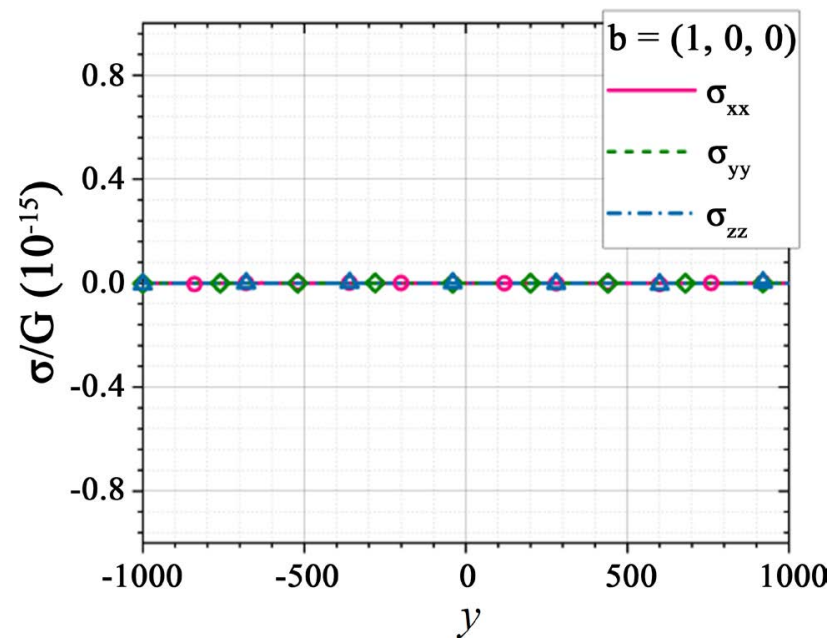

Figure 8. Comparison of analytical solutions (solid and dashed lines) to the results of the numerical "collocation point" method (symbols) along $y$-direction for non-zero $b_{x}$.

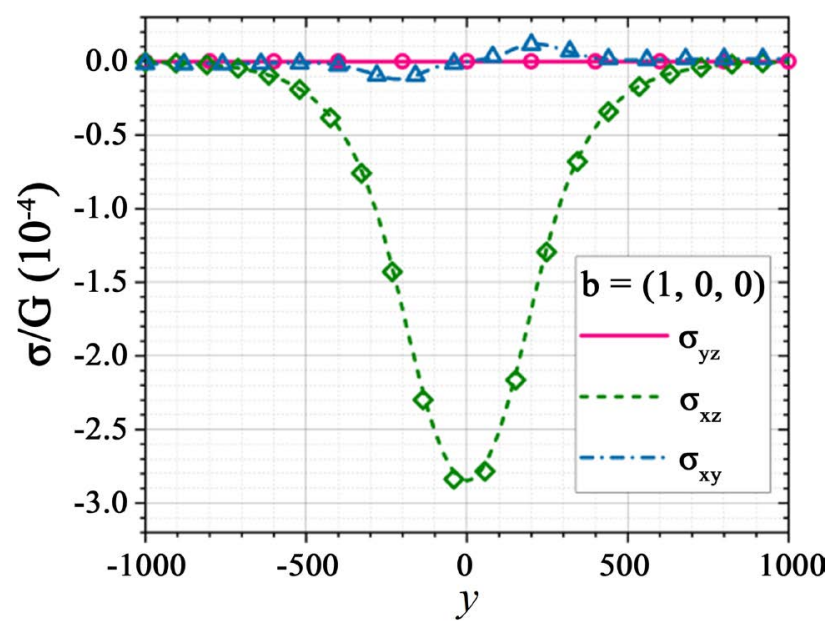

Figure 9. Comparison of analytical solutions (solid and dashed lines) to the results of the numerical "collocation point" method (symbols) along $y$-direction for non-zero $b_{x}$.

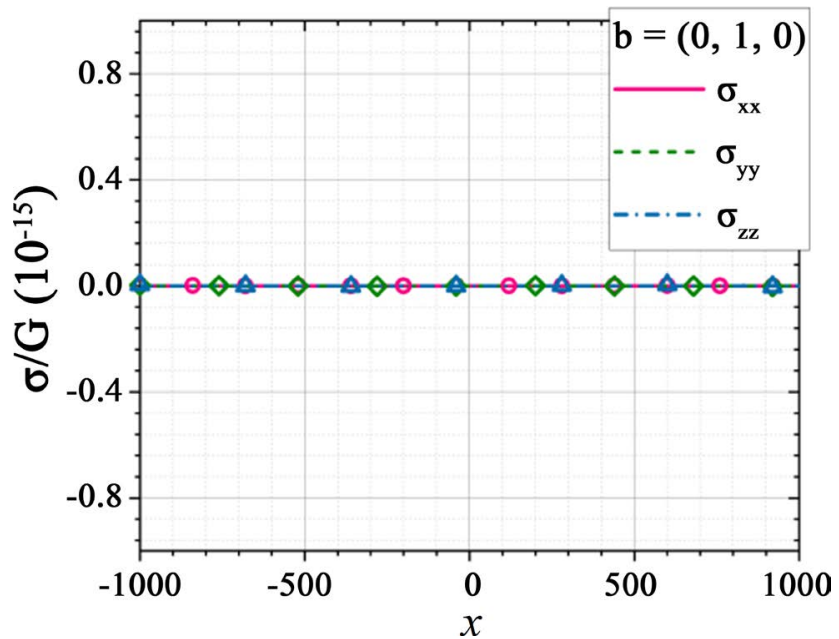

Figure 10. Comparison of analytical solutions (solid and dashed lines) to the results of the numerical "collocation point" method (symbols) along $x$-direction for non-zero $b_{y}$. 


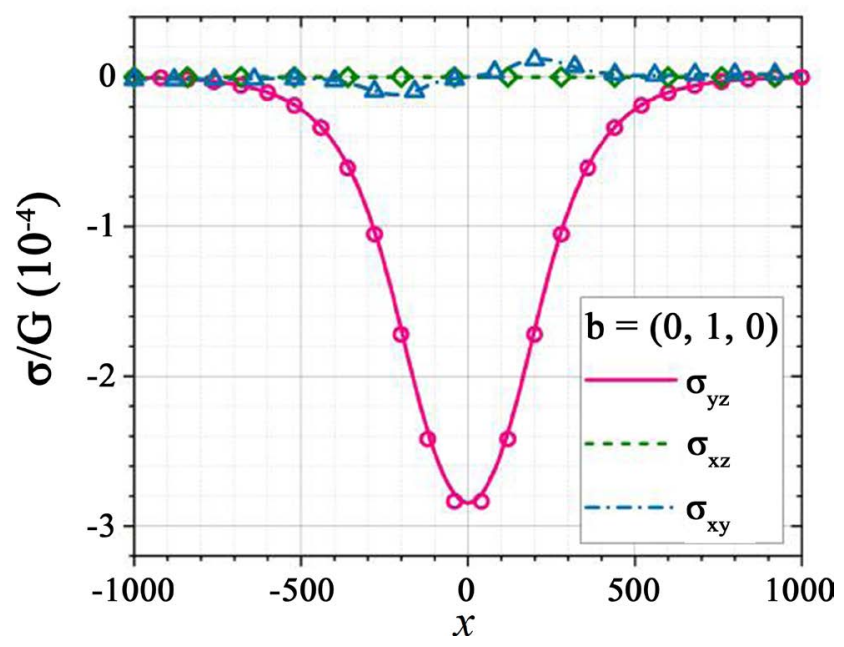

Figure 11. Comparison of analytical solutions (solid and dashed lines) to the results of the numerical "collocation point" method (symbols) along $x$-direction for non-zero $b_{y}$.

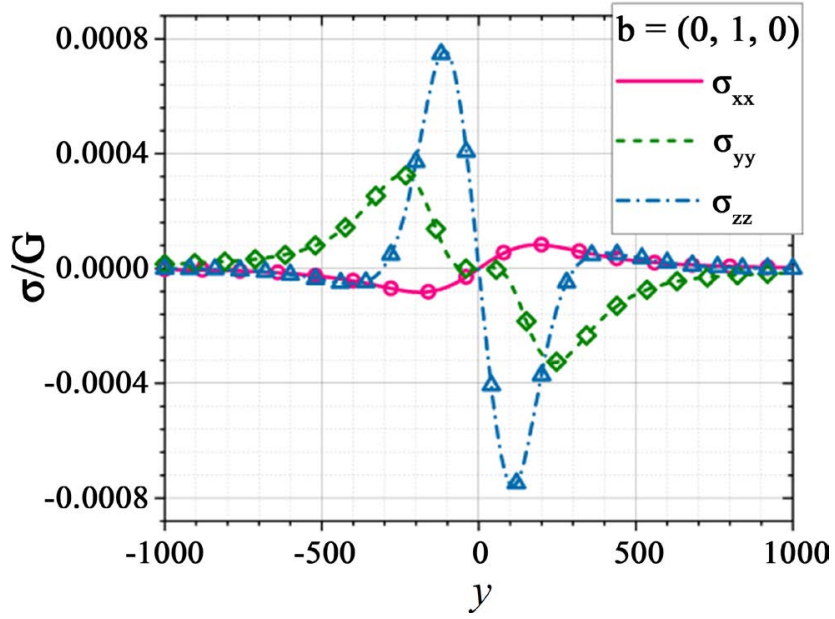

Figure 12. Comparison of analytical solutions (solid and dashed lines) to the results of the numerical "collocation point" method (symbols) along $y$-direction for non-zero $b_{y}$.

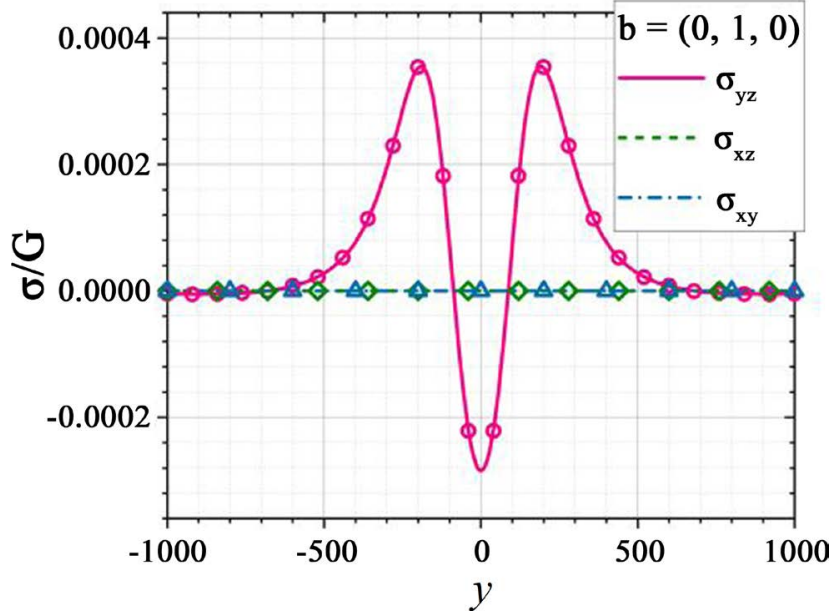

Figure 13. Comparison of analytical solutions (solid and dashed lines) to the results of the numerical "collocation point" method (symbols) along $y$-direction for non-zero $b_{y}$. 


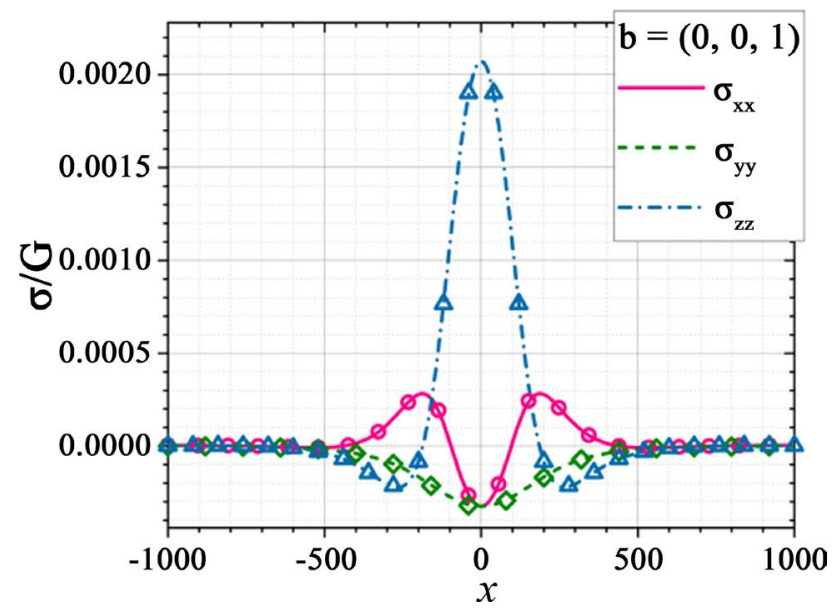

Figure 14. Comparison of analytical solutions (solid and dashed lines) to the results of the numerical "collocation point" method (symbols) along $x$-direction for non-zero $b_{z}$.

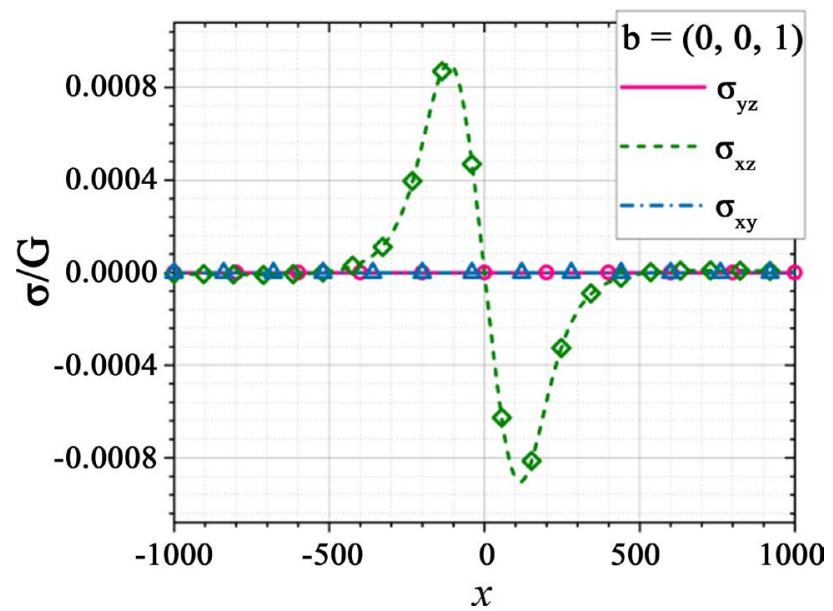

Figure 15. Comparison of analytical solutions (solid and dashed lines) to the results of the numerical "collocation point" method (symbols) along $x$-direction for non-zero $b_{z}$.

The figures show perfect match between the analytical and numerical solutions.

\section{Conclusions}

In conclusion, the strain and stress fields associated with a sub-surface rectangular dislocation loop that is parallel to the free surface of a semi-infinite solid have been developed. These are obtained from three different contributions or terms: a term associated with the loop being in an infinite medium, another term associated with an opposite Burgers vector image loop, and the third from surface correction terms. The infinite terms and the surface correction terms were developed here.

The developed field solutions were verified using analytical equations and numerical comparisons. The verifications were to ensure satisfaction of the zero traction boundary condition on the free surface, the satisfaction of the equilibrium equations, the satisfaction of the strain compatibility equations, and compari- 
sons against numerical results from the proven "collocation point" numerical method.

\section{Conflicts of Interest}

The authors declare no conflicts of interest regarding the publication of this paper.

\section{References}

[1] Hull, D. and Bacon, D.J. (2011) Introduction to Dislocations. Butterworth-Heinemann, Oxford. https://doi.org/10.1016/C2009-0-64358-0

[2] Hirth, J.P. and Lothe, J. (1982) Theory of Dislocations. Krieger Publishing Company, Malabar.

[3] Weertman, J. and Weertman, J.R. (1992) Elementary Dislocation Theory. Oxford University Press, Oxford.

[4] Kroupa, F. (1960) Circular Edge Dislocation Loop. Cechoslovackij fiziceskij zurnal $B, 10,284-293$. https://doi.org/10.1007/BF02033533

[5] Bullough, R. and Newman, R.C. (1960) The Spacing of Prismatic Dislocation Loops. The Philosophical Magazine, 5, 921-926.

https://doi.org/10.1080/14786436008238311

[6] Keller, J.M. (1957) Unpublished.

[7] Kröner, E. (1958) Kontinuumstheorie Der Versetzungen Und Eigenspannungen. Springer-Verlag, Berlin.

[8] Kroupa, F. (1962) Interaction between Prismatic Dislocation Loops and Straight Dislocations. Part I. The Philosophical Magazine, 7, 783-801. https://doi.org/10.1080/14786436208212669

[9] Marcinkowski, M.J. and Sree Harsha, K.S. (1968) Properties of Finite Circular Dislocation Glide Loops. Journal of Applied Physics, 39, 1775-1783.

https://doi.org/10.1063/1.1656429

[10] Khraishi, T.A., Hirth, J.P., Zbib, H.M. and Khaleel, M.A. (2000) The Displacement, and Strain-Stress Fields of a General Circular Volterra Dislocation Loop. International Journal of Engineering Science, 5, 251-266. https://doi.org/10.1016/S0020-7225(99)00038-5

[11] Khraishi, T.A., Zbib, H.M., Hirth, J.P. and de la Rubia, T.D. (2000) The Stress Field of a General Circular Volterra Dislocation Loop: Analytical and Numerical Approaches. Philosophical Magazine Letters, 80, 95-105. https://doi.org/10.1080/095008300176353

[12] Khraishi, T.A. and Zbib, H.M. (2002) The Displacement Field of a Rectangular Volterra Dislocation Loop. Philosophical Magazine Letters, 82, 265-277. https://doi.org/10.1080/09500830210125770

[13] Demir, I., Hirth, J.P. and Zbib, H.M. (1992) Extended Stress Field around a Cylindrical Crack Using the Theory of Dislocation Pile-Ups. International Journal of Engineering Science, 30, 829-845. https://doi.org/10.1016/0020-7225(92)90013-7

[14] Demir, I., Hirth, J.P. and Zbib, H.M. (1992) The Somigliana Ring Dislocation. Journal of Elasticity, 28, 223-246. https://doi.org/10.1007/BF00132212

[15] Demir, I. and Khraishi, T.A. (2005) The Torsional Dislocation Loop and Mode III Cylindrical Crack. Journal of Mechanics, 21, 109-116. https://doi.org/10.1017/S1727719100004585 
[16] Yoffe, E.H. (1961) A Dislocation at a Free Surface. The Philosophical Magazine, 6, 1147-1155. https://doi.org/10.1080/14786436108239675

[17] Baštecká, J. (1964) Interaction of Dislocation Loop with Free Surface. Cechoslovackij Fiziceskij Zurnal B, 14, 430-442. https://doi.org/10.1007/BF01689476

[18] Chou, Y.T. (1963) Energy of Circular Dislocation Loops in Thin Plates. Acta MetalIurgica, 13, 829-834. https://doi.org/10.1016/0001-6160(63)90051-8

[19] Baštecká, J. and Kroupa, F. (1964) Elastic Interaction of Dislocation Loops and Point Defects. Cechoslovackij Fiziceskij Zurnal B, 14, 443-453.

https://doi.org/10.1007/BF01689477

[20] Bacon, D.J. and Groves, P.P. (1970) The Dislocation in a Semi-Infinite Isotropic Medium. In: Bacon, D.J. and Groves, P.P., Ed., Fundamental Aspects of Dislocation Theory, Witt, R. and Bullough, R., Inc., Washington DC, 35-45.

[21] Groves, P.P. and Bacon, D.J. (1970) The Dislocation Loop Near a Free Surface. Philosophical Magazine, 22, 83-91. https://doi.org/10.1080/14786437008228153

[22] Jing, P., Khraishi, T.A., Zepeda-Ruiz, L.A. and Wirth, B.D. (2009) The Elastic Fields of Sub-Surface Dislocation Loops: A Comparison between Analytical ContinuumTheory Solutions and Atomistic Calculations. International Journal of Theoretical and Applied Multiscale Mechanics, 1, 71-85.

https://doi.org/10.1504/IJTAMM.2009.022472

[23] Maurissen, Y. and Capella, L. (1974) Stress Field of a Dislocation Segment Parallel to a Free Surface. Philosophical Magazine, 29, 1227-1229.

https://doi.org/10.1080/14786437408226608

[24] Maurissen, Y. and Capella, L. (1974) Stress Field of a Dislocation Segment Perpendicular to a Free Surface. Philosophical Magazine, 30, 679-683.

https://doi.org/10.1080/14786439808206591

[25] Comninou, M. and Dundurs, J. (1975) The Angular Dislocation in a Half Space. Journal of Elasticity, 19, 203-216. https://doi.org/10.1007/BF00126985

[26] Lothe, J., Indenbom, V.L. and Chamrov, V.A. (1982) Elastic Field and Self-Force of Dislocations Emerging at the Free Surfaces of an Anisotropic Half-Space. Phys. Status Solidi (B), 20, 671-677. https://doi.org/10.1002/pssb.2221110231

[27] Gosling, T.J. and Willis, J.R. (1994) A Line-Integral Representation for the Stresses Due to an Arbitrary Dislocation in an Isotropic Half-Space. Journal of the Mechanics and Physics of Solids, 42, 1199-1221. https://doi.org/10.1016/0022-5096(94)90032-9

[28] Khraishi, T. A., Zbib, H. M. and de la Rubia, T. D. (2001) The Treatment of Traction free Boundary Condition in Three-Dimensional Dislocation Dynamics using Generalized Image Stress Analysis. Materials Science and Engineering. A, 309-310, 283-287. https://doi.org/10.1016/S0921-5093(00)01727-5

[29] Khraishi, T. A. and Zbib, H. M. (2002) Free Surface Effects in 3D Dislocation Dynamics: Formulation and Modeling. Journal of Engineering Materials and Technology, 124, 342-351. https://doi.org/10.1115/1.1479694

[30] Yan, L., Khraishi, T. A., Shen, Y.-L. and Horstemeyer, M. F. (2004,) A Distributed Dislocation Method for Treating Free-Surface Image Stresses in 3D Dislocation Dynamics Simulations. Modelling and Simulation in Materials Science and Engineering, 12, 289-301. https://doi.org/10.1088/0965-0393/12/4/S01

[31] Siddique, A.B. and Khraishi, T. A. (2020) Numerical Methodology for Treating Static and Dynamic Dislocation Problems Near a Free Surface. Journal of Physics Communications, 4, Article ID: 055005. https://doi.org/10.1088/2399-6528/ab8ff9

[32] Lerma, J.D., Khraishi, T. and Shen, Y.L. (2007) Elastic Fields of 2D and 3D Misfit 
Particles in an Infinite Medium. Mechanics Research Communications, 34, 31-43. https://doi.org/10.1016/j.mechrescom.2006.06.002

[33] Lerma, J., Khraishi, T., Kataria, S. and Shen, Y.L. (2015) Distributed Dislocation Method for Determining Elastic Fields of 2D and 3D Volume Misfit Particles in Infinite Space and Extension of the Method for Particles in Half Space. Journal of Mechanics, 31, 249-260. https://doi.org/10.1017/jmech.2014.85

[34] Khraishi, T. A. and Shen, Y.-L. (2011) Introductory Continuum Mechanics with Applications to Elasticity. University Readers/Cognella, San Diego. 


\section{Appendix A}

The surface correction terms for stress considering only $b_{x}$ (the $x$-component of the Burgers vector):

$$
\begin{aligned}
& \frac{\sigma_{x x}}{\mu}=\frac{1}{2 K \pi} b_{x} c\left(K _ { 2 } \left(\frac{Q_{3}}{\sqrt{A_{2}} B_{1}}-\frac{Q_{3}}{\sqrt{A_{4}} B_{2}}-\frac{2 Q_{1}^{2} Q_{3}}{\sqrt{A_{2}} B_{1}^{2}}-\frac{Q_{1}^{2} Q_{3}}{A_{2}^{3 / 2} B_{1}}+\frac{2 Q_{2}^{2} Q_{3}}{\sqrt{A_{4}} B_{2}^{2}}+\frac{Q_{2}^{2} Q_{3}}{A_{4}^{3 / 2} B_{2}}\right.\right. \\
& \left.-\frac{Q_{4}}{\sqrt{A_{1}} B_{1}}+\frac{Q_{4}}{\sqrt{A_{3}} B_{2}}+\frac{2 Q_{1}^{2} Q_{4}}{\sqrt{A_{1}} B_{1}^{2}}+\frac{Q_{1}^{2} Q_{4}}{A_{1}^{3 / 2} B_{1}}-\frac{2 Q_{2}^{2} Q_{4}}{\sqrt{A_{3}} B_{2}^{2}}-\frac{Q_{2}^{2} Q_{4}}{A_{3}^{3 / 2} B_{2}}\right)+\frac{6 p Q_{1}^{2} Q_{3} z}{A_{2}^{3 / 2} B_{1}^{2}} \\
& -\frac{6 p Q_{2}^{2} Q_{3} z}{A_{4}^{3 / 2} B_{2}^{2}}-\frac{6 p Q_{1}^{2} Q_{4} z}{A_{1}^{3 / 2} B_{1}^{2}}+\frac{6 p Q_{2}^{2} Q_{4} z}{A_{3}^{3 / 2} B_{2}^{2}}-\frac{p Q_{4}\left(3 A_{1}-Q_{4}^{2}\right) z}{A_{1}^{3 / 2} B_{1}^{2}} \\
& +\frac{4 p Q_{1}^{2} Q_{4}\left(3 A_{1}-Q_{4}^{2}\right) z}{A_{1}^{3 / 2} B_{1}^{3}}+\frac{3 p Q_{1}^{2} Q_{4}\left(3 A_{1}-Q_{4}^{2}\right) z}{A_{1}^{5 / 2} B_{1}^{2}}+\frac{p Q_{4}\left(3 A_{3}-Q_{4}^{2}\right) z}{A_{3}^{3 / 2} B_{2}^{2}} \\
& -\frac{4 p Q_{2}^{2} Q_{4}\left(3 A_{3}-Q_{4}^{2}\right) z}{A_{3}^{3 / 2} B_{2}^{3}}-\frac{3 p Q_{2}^{2} Q_{4}\left(3 A_{3}-Q_{4}^{2}\right) z}{A_{3}^{5 / 2} B_{2}^{2}}+\frac{p Q_{3} S_{1} z}{A_{2}^{3 / 2} B_{1}^{2}}-\frac{4 p Q_{1}^{2} Q_{3} S_{1} z}{A_{2}^{3 / 2} B_{1}^{3}} \\
& \left.-\frac{3 p Q_{1}^{2} Q_{3} S_{1} z}{A_{2}^{5 / 2} B_{1}^{2}}-\frac{p Q_{3} S_{2} z}{A_{4}^{3 / 2} B_{2}^{2}}+\frac{4 p Q_{2}^{2} Q_{3} S_{2} z}{A_{4}^{3 / 2} B_{2}^{3}}+\frac{3 p Q_{2}^{2} Q_{3} S_{2} z}{A_{4}^{5 / 2} B_{2}^{2}}\right) \\
& -\frac{1}{K_{2}} 2 v\left(-\frac{1}{4 K \pi} b_{x} c\left(-\frac{K_{2} Q_{3}}{\sqrt{A_{2}} B_{1}}+\frac{K_{2} Q_{3}}{\sqrt{A_{4}} B_{2}}-\frac{2 K_{4} p Q_{3}}{\sqrt{A_{2}} B_{1}^{2}}-\frac{K_{4} p Q_{3}}{A_{2}^{3 / 2} B_{1}}+\frac{2 K_{4} p Q_{3}}{\sqrt{A_{4}} B_{2}^{2}}\right.\right. \\
& +\frac{K_{4} p Q_{3}}{A_{4}^{3 / 2} B_{2}}+\frac{K_{2} Q_{4}}{\sqrt{A_{1}} B_{1}}-\frac{K_{2} Q_{4}}{\sqrt{A_{3}} B_{2}}+\frac{2 K_{4} p Q_{4}}{\sqrt{A_{1}} B_{1}^{2}}+\frac{K_{4} p Q_{4}}{A_{1}^{3 / 2} B_{1}}-\frac{2 K_{4} p Q_{4}}{\sqrt{A_{3}} B_{2}^{2}}-\frac{K_{4} p Q_{4}}{A_{3}^{3 / 2} B_{2}} \\
& -\frac{p^{2} Q_{4}\left(3 A_{1}-Q_{4}^{2}\right)}{A_{1}^{3 / 2} B_{1}^{2}}+\frac{p^{2} Q_{4}\left(3 A_{3}-Q_{4}^{2}\right)}{A_{3}^{3 / 2} B_{2}^{2}}+\frac{p^{2} Q_{3} S_{1}}{A_{2}^{3 / 2} B_{1}^{2}}-\frac{p^{2} Q_{3} S_{2}}{A_{4}^{3 / 2} B_{2}^{2}}+\frac{6 p^{3} Q_{3} z}{A_{2}^{3 / 2} B_{1}^{2}} \\
& -\frac{6 p^{3} Q_{3} z}{A_{4}^{3 / 2} B_{2}^{2}}-\frac{6 p^{3} Q_{4} z}{A_{1}^{3 / 2} B_{1}^{2}}+\frac{6 p^{3} Q_{4} z}{A_{3}^{3 / 2} B_{2}^{2}}-\frac{2 p Q_{4}\left(3 A_{1}-Q_{4}^{2}\right) z}{A_{1}^{3 / 2} B_{1}^{2}}+\frac{4 p^{3} Q_{4}\left(3 A_{1}-Q_{4}^{2}\right) z}{A_{1}^{3 / 2} B_{1}^{3}} \\
& +\frac{3 p^{3} Q_{4}\left(3 A_{1}-Q_{4}^{2}\right) z}{A_{1}^{5 / 2} B_{1}^{2}}+\frac{2 p Q_{4}\left(3 A_{3}-Q_{4}^{2}\right) z}{A_{3}^{3 / 2} B_{2}^{2}}-\frac{4 p^{3} Q_{4}\left(3 A_{3}-Q_{4}^{2}\right) z}{A_{3}^{3 / 2} B_{2}^{3}} \\
& -\frac{3 p^{3} Q_{4}\left(3 A_{3}-Q_{4}^{2}\right) z}{A_{3}^{5 / 2} B_{2}^{2}}+\frac{2 p Q_{3} S_{1} z}{A_{2}^{3 / 2} B_{1}^{2}}-\frac{4 p^{3} Q_{3} S_{1} z}{A_{2}^{3 / 2} B_{1}^{3}}-\frac{3 p^{3} Q_{3} S_{1} z}{A_{2}^{5 / 2} B_{1}^{2}}-\frac{2 p Q_{3} S_{2} z}{A_{4}^{3 / 2} B_{2}^{2}} \\
& \left.+\frac{4 p^{3} Q_{3} S_{2} z}{A_{4}^{3 / 2} B_{2}^{3}}+\frac{3 p^{3} Q_{3} S_{2} z}{A_{4}^{5 / 2} B_{2}^{2}}\right)-\frac{1}{4 K \pi} b_{x} c\left(K _ { 2 } \left(\frac{Q_{3}}{\sqrt{A_{2}} B_{1}}-\frac{Q_{3}}{\sqrt{A_{4}} B_{2}}-\frac{2 Q_{1}^{2} Q_{3}}{\sqrt{A_{2}} B_{1}^{2}}\right.\right. \\
& -\frac{Q_{1}^{2} Q_{3}}{A_{2}^{3 / 2} B_{1}}+\frac{2 Q_{2}^{2} Q_{3}}{\sqrt{A_{4}} B_{2}^{2}}+\frac{Q_{2}^{2} Q_{3}}{A_{4}^{3 / 2} B_{2}}-\frac{Q_{4}}{\sqrt{A_{1}} B_{1}}+\frac{Q_{4}}{\sqrt{A_{3}} B_{2}}+\frac{2 Q_{1}^{2} Q_{4}}{\sqrt{A_{1}} B_{1}^{2}}+\frac{Q_{1}^{2} Q_{4}}{A_{1}^{3 / 2} B_{1}} \\
& \left.-\frac{2 Q_{2}^{2} Q_{4}}{\sqrt{A_{3}} B_{2}^{2}}-\frac{Q_{2}^{2} Q_{4}}{A_{3}^{3 / 2} B_{2}}\right)+\frac{6 p Q_{1}^{2} Q_{3} z}{A_{2}^{3 / 2} B_{1}^{2}}-\frac{6 p Q_{2}^{2} Q_{3} z}{A_{4}^{3 / 2} B_{2}^{2}}-\frac{6 p Q_{1}^{2} Q_{4} z}{A_{1}^{3 / 2} B_{1}^{2}}+\frac{6 p Q_{2}^{2} Q_{4} z}{A_{3}^{3 / 2} B_{2}^{2}} \\
& -\frac{p Q_{4}\left(3 A_{1}-Q_{4}^{2}\right) z}{A_{1}^{3 / 2} B_{1}^{2}}+\frac{4 p Q_{1}^{2} Q_{4}\left(3 A_{1}-Q_{4}^{2}\right) z}{A_{1}^{3 / 2} B_{1}^{3}}+\frac{3 p Q_{1}^{2} Q_{4}\left(3 A_{1}-Q_{4}^{2}\right) z}{A_{1}^{5 / 2} B_{1}^{2}} \\
& +\frac{p Q_{4}\left(3 A_{3}-Q_{4}^{2}\right) z}{A_{3}^{3 / 2} B_{2}^{2}}-\frac{4 p Q_{2}^{2} Q_{4}\left(3 A_{3}-Q_{4}^{2}\right) z}{A_{3}^{3 / 2} B_{2}^{3}}-\frac{3 p Q_{2}^{2} Q_{4}\left(3 A_{3}-Q_{4}^{2}\right) z}{A_{3}^{5 / 2} B_{2}^{2}}
\end{aligned}
$$




$$
\begin{aligned}
& +\frac{p Q_{3} S_{1} z}{A_{2}^{3 / 2} B_{1}^{2}}-\frac{4 p Q_{1}^{2} Q_{3} S_{1} z}{A_{2}^{3 / 2} B_{1}^{3}}-\frac{3 p Q_{1}^{2} Q_{3} S_{1} z}{A_{2}^{5 / 2} B_{1}^{2}}-\frac{p Q_{3} S_{2} z}{A_{4}^{3 / 2} B_{2}^{2}}+\frac{4 p Q_{2}^{2} Q_{3} S_{2} z}{A_{4}^{3 / 2} B_{2}^{3}} \\
& \left.+\frac{3 p Q_{2}^{2} Q_{3} S_{2} z}{A_{4}^{5 / 2} B_{2}^{2}}\right)+\frac{b_{x} c}{-4 \pi K}\left(K_{2}\left(\frac{Q_{3}}{A_{2}^{3 / 2}}-\frac{Q_{3}}{A_{4}^{3 / 2}}-\frac{Q_{4}}{A_{1}^{3 / 2}}+\frac{Q_{4}}{A_{3}^{3 / 2}}\right)+\frac{3 p Q_{3} z}{A_{2}^{5 / 2}}\right. \\
& \left.\left.-\frac{3 p Q_{3} z}{A_{4}^{5 / 2}}-\frac{3 p Q_{4} z}{A_{1}^{5 / 2}}+\frac{3 p Q_{4} z}{A_{3}^{5 / 2}}\right)\right) \\
& \frac{\sigma_{y y}}{\mu}=\frac{b_{x}}{2 K \pi} c\left(K_{2}\left(\frac{Q_{3}}{A_{2}^{3 / 2}}-\frac{Q_{3}}{A_{4}^{3 / 2}}-\frac{Q_{4}}{A_{1}^{3 / 2}}+\frac{Q_{4}}{A_{3}^{3 / 2}}\right)+\frac{3 p Q_{3} z}{A_{2}^{5 / 2}}-\frac{3 p Q_{3} z}{A_{4}^{5 / 2}}-\frac{3 p Q_{4} z}{A_{1}^{5 / 2}}\right. \\
& \left.+\frac{3 p Q_{4} z}{A_{3}^{5 / 2}}\right)-\frac{1}{K_{2}} 2 v\left(-\frac{1}{4 K \pi} b_{x} c\left(-\frac{K_{2} Q_{3}}{\sqrt{A_{2}} B_{1}}+\frac{K_{2} Q_{3}}{\sqrt{A_{4}} B_{2}}-\frac{2 K_{4} p Q_{3}}{\sqrt{A_{2}} B_{1}^{2}}-\frac{K_{4} p Q_{3}}{A_{2}^{3 / 2} B_{1}}\right.\right. \\
& +\frac{2 K_{4} p Q_{3}}{\sqrt{A_{4}} B_{2}^{2}}+\frac{K_{4} p Q_{3}}{A_{4}^{3 / 2} B_{2}}+\frac{K_{2} Q_{4}}{\sqrt{A_{1}} B_{1}}-\frac{K_{2} Q_{4}}{\sqrt{A_{3}} B_{2}}+\frac{2 K_{4} p Q_{4}}{\sqrt{A_{1}} B_{1}^{2}}+\frac{K_{4} p Q_{4}}{A_{1}^{3 / 2} B_{1}}-\frac{2 K_{4} p Q_{4}}{\sqrt{A_{3}} B_{2}^{2}} \\
& -\frac{K_{4} p Q_{4}}{A_{3}^{3 / 2} B_{2}}-\frac{p^{2} Q_{4}\left(3 A_{1}-Q_{4}^{2}\right)}{A_{1}^{3 / 2} B_{1}^{2}}+\frac{p^{2} Q_{4}\left(3 A_{3}-Q_{4}^{2}\right)}{A_{3}^{3 / 2} B_{2}^{2}}+\frac{p^{2} Q_{3} S_{1}}{A_{2}^{3 / 2} B_{1}^{2}}-\frac{p^{2} Q_{3} S_{2}}{A_{4}^{3 / 2} B_{2}^{2}} \\
& +\frac{6 p^{3} Q_{3} z}{A_{2}^{3 / 2} B_{1}^{2}}-\frac{6 p^{3} Q_{3} z}{A_{4}^{3 / 2} B_{2}^{2}}-\frac{6 p^{3} Q_{4} z}{A_{1}^{3 / 2} B_{1}^{2}}+\frac{6 p^{3} Q_{4} z}{A_{3}^{3 / 2} B_{2}^{2}}-\frac{2 p Q_{4}\left(3 A_{1}-Q_{4}^{2}\right) z}{A_{1}^{3 / 2} B_{1}^{2}} \\
& +\frac{4 p^{3} Q_{4}\left(3 A_{1}-Q_{4}^{2}\right) z}{A_{1}^{3 / 2} B_{1}^{3}}+\frac{3 p^{3} Q_{4}\left(3 A_{1}-Q_{4}^{2}\right) z}{A_{1}^{5 / 2} B_{1}^{2}}+\frac{2 p Q_{4}\left(3 A_{3}-Q_{4}^{2}\right) z}{A_{3}^{3 / 2} B_{2}^{2}} \\
& -\frac{4 p^{3} Q_{4}\left(3 A_{3}-Q_{4}^{2}\right) z}{A_{3}^{3 / 2} B_{2}^{3}}-\frac{3 p^{3} Q_{4}\left(3 A_{3}-Q_{4}^{2}\right) z}{A_{3}^{5 / 2} B_{2}^{2}}+\frac{2 p Q_{3} S_{1} z}{A_{2}^{3 / 2} B_{1}^{2}}-\frac{4 p^{3} Q_{3} S_{1} z}{A_{2}^{3 / 2} B_{1}^{3}} \\
& \left.-\frac{3 p^{3} Q_{3} S_{1} z}{A_{2}^{5 / 2} B_{1}^{2}}-\frac{2 p Q_{3} S_{2} z}{A_{4}^{3 / 2} B_{2}^{2}}+\frac{4 p^{3} Q_{3} S_{2} z}{A_{4}^{3 / 2} B_{2}^{3}}+\frac{3 p^{3} Q_{3} S_{2} z}{A_{4}^{5 / 2} B_{2}^{2}}\right) \\
& -\frac{1}{4 K \pi} b_{x} c\left(K _ { 2 } \left(\frac{Q_{3}}{\sqrt{A_{2}} B_{1}}-\frac{Q_{3}}{\sqrt{A_{4}} B_{2}}-\frac{2 Q_{1}^{2} Q_{3}}{\sqrt{A_{2}} B_{1}^{2}}-\frac{Q_{1}^{2} Q_{3}}{A_{2}^{3 / 2} B_{1}}+\frac{2 Q_{2}^{2} Q_{3}}{\sqrt{A_{4}} B_{2}^{2}}\right.\right. \\
& \left.+\frac{Q_{2}^{2} Q_{3}}{A_{4}^{3 / 2} B_{2}}-\frac{Q_{4}}{\sqrt{A_{1}} B_{1}}+\frac{Q_{4}}{\sqrt{A_{3}} B_{2}}+\frac{2 Q_{1}^{2} Q_{4}}{\sqrt{A_{1}} B_{1}^{2}}+\frac{Q_{1}^{2} Q_{4}}{A_{1}^{3 / 2} B_{1}}-\frac{2 Q_{2}^{2} Q_{4}}{\sqrt{A_{3}} B_{2}^{2}}-\frac{Q_{2}^{2} Q_{4}}{A_{3}^{3 / 2} B_{2}}\right) \\
& +\frac{6 p Q_{1}^{2} Q_{3} z}{A_{2}^{3 / 2} B_{1}^{2}}-\frac{6 p Q_{2}^{2} Q_{3} z}{A_{4}^{3 / 2} B_{2}^{2}}-\frac{6 p Q_{1}^{2} Q_{4} z}{A_{1}^{3 / 2} B_{1}^{2}}+\frac{6 p Q_{2}^{2} Q_{4} z}{A_{3}^{3 / 2} B_{2}^{2}}-\frac{p Q_{4}\left(3 A_{1}-Q_{4}^{2}\right) z}{A_{1}^{3 / 2} B_{1}^{2}} \\
& +\frac{4 p Q_{1}^{2} Q_{4}\left(3 A_{1}-Q_{4}^{2}\right) z}{A_{1}^{3 / 2} B_{1}^{3}}+\frac{3 p Q_{1}^{2} Q_{4}\left(3 A_{1}-Q_{4}^{2}\right) z}{A_{1}^{5 / 2} B_{1}^{2}}+\frac{p Q_{4}\left(3 A_{3}-Q_{4}^{2}\right) z}{A_{3}^{3 / 2} B_{2}^{2}} \\
& -\frac{4 p Q_{2}^{2} Q_{4}\left(3 A_{3}-Q_{4}^{2}\right) z}{A_{3}^{3 / 2} B_{2}^{3}}-\frac{3 p Q_{2}^{2} Q_{4}\left(3 A_{3}-Q_{4}^{2}\right) z}{A_{3}^{5 / 2} B_{2}^{2}}+\frac{p Q_{3} S_{1} z}{A_{2}^{3 / 2} B_{1}^{2}}-\frac{4 p Q_{1}^{2} Q_{3} S_{1} z}{A_{2}^{3 / 2} B_{1}^{3}} \\
& \left.-\frac{3 p Q_{1}^{2} Q_{3} S_{1} z}{A_{2}^{5 / 2} B_{1}^{2}}-\frac{p Q_{3} S_{2} z}{A_{4}^{3 / 2} B_{2}^{2}}+\frac{4 p Q_{2}^{2} Q_{3} S_{2} z}{A_{4}^{3 / 2} B_{2}^{3}}+\frac{3 p Q_{2}^{2} Q_{3} S_{2} z}{A_{4}^{5 / 2} B_{2}^{2}}\right)-\frac{b_{x} c}{4 \pi K}\left(K _ { 2 } \left(\frac{Q_{3}}{A_{2}^{3 / 2}}\right.\right. \\
& \left.\left.\left.-\frac{Q_{3}}{A_{4}^{3 / 2}}-\frac{Q_{4}}{A_{1}^{3 / 2}}+\frac{Q_{4}}{A_{3}^{3 / 2}}\right)+\frac{3 p Q_{3} z}{A_{2}^{5 / 2}}-\frac{3 p Q_{3} z}{A_{4}^{5 / 2}}-\frac{3 p Q_{4} z}{A_{1}^{5 / 2}}+\frac{3 p Q_{4} z}{A_{3}^{5 / 2}}\right)\right)
\end{aligned}
$$




$$
\begin{aligned}
& \frac{\sigma_{z z}}{\mu}=\frac{1}{2 K \pi} b_{x} c\left(-\frac{K_{2} Q_{3}}{\sqrt{A_{2}} B_{1}}+\frac{K_{2} Q_{3}}{\sqrt{A_{4}} B_{2}}-\frac{2 G_{1} p Q_{3}}{\sqrt{A_{2}} B_{1}^{2}}-\frac{G_{1} p Q_{3}}{A_{2}^{3 / 2} B_{1}}+\frac{2 G_{1} p Q_{3}}{\sqrt{A_{4}} B_{2}^{2}}+\frac{G_{1} p Q_{3}}{A_{4}^{3 / 2} B_{2}}\right. \\
& +\frac{K_{2} Q_{4}}{\sqrt{A_{1}} B_{1}}-\frac{K_{2} Q_{4}}{\sqrt{A_{3}} B_{2}}+\frac{2 G_{1} p Q_{4}}{\sqrt{A_{1}} B_{1}^{2}}+\frac{G_{1} p Q_{4}}{A_{1}^{3 / 2} B_{1}}-\frac{2 G_{1} p Q_{4}}{\sqrt{A_{3}} B_{2}^{2}}-\frac{G_{1} p Q_{4}}{A_{3}^{3 / 2} B_{2}} \\
& -\frac{p^{2} Q_{4}\left(3 A_{1}-Q_{4}^{2}\right)}{A_{1}^{3 / 2} B_{1}^{2}}+\frac{p^{2} Q_{4}\left(3 A_{3}-Q_{4}^{2}\right)}{A_{3}^{3 / 2} B_{2}^{2}}+\frac{p^{2} Q_{3} S_{1}}{A_{2}^{3 / 2} B_{1}^{2}}-\frac{p^{2} Q_{3} S_{2}}{A_{4}^{3 / 2} B_{2}^{2}}+\frac{6 p^{3} Q_{3} z}{A_{2}^{3 / 2} B_{1}^{2}} \\
& -\frac{6 p^{3} Q_{3} z}{A_{4}^{3 / 2} B_{2}^{2}}-\frac{6 p^{3} Q_{4} z}{A_{1}^{3 / 2} B_{1}^{2}}+\frac{6 p^{3} Q_{4} z}{A_{3}^{3 / 2} B_{2}^{2}}-\frac{2 p Q_{4}\left(3 A_{1}-Q_{4}^{2}\right) z}{A_{1}^{3 / 2} B_{1}^{2}}+\frac{4 p^{3} Q_{4}\left(3 A_{1}-Q_{4}^{2}\right) z}{A_{1}^{3 / 2} B_{1}^{3}} \\
& +\frac{3 p^{3} Q_{4}\left(3 A_{1}-Q_{4}^{2}\right) z}{A_{1}^{5 / 2} B_{1}^{2}}+\frac{2 p Q_{4}\left(3 A_{3}-Q_{4}^{2}\right) z}{A_{3}^{3 / 2} B_{2}^{2}}-\frac{4 p^{3} Q_{4}\left(3 A_{3}-Q_{4}^{2}\right) z}{A_{3}^{3 / 2} B_{2}^{3}} \\
& -\frac{3 p^{3} Q_{4}\left(3 A_{3}-Q_{4}^{2}\right) z}{A_{3}^{5 / 2} B_{2}^{2}}+\frac{2 p Q_{3} S_{1} z}{A_{2}^{3 / 2} B_{1}^{2}}--\frac{4 p^{3} Q_{3} S_{1} z}{A_{2}^{3 / 2} B_{1}^{3}}-\frac{3 p^{3} Q_{3} S_{1} z}{A_{2}^{5 / 2} B_{1}^{2}}-\frac{2 p Q_{3} S_{2} z}{A_{4}^{3 / 2} B_{2}^{2}} \\
& \left.+\frac{4 p^{3} Q_{3} S_{2} z}{A_{4}^{3 / 2} B_{2}^{3}}+\frac{3 p^{3} Q_{3} S_{2} z}{A_{4}^{5 / 2} B_{2}^{2}}\right)-\frac{1}{K_{2}} 2 v\left(-\frac{1}{4 K \pi} b_{x} c\left(-\frac{K_{2} Q_{3}}{\sqrt{A_{2}} B_{1}}+\frac{K_{2} Q_{3}}{\sqrt{A_{4}} B_{2}}\right.\right. \\
& -\frac{2 K_{4} p Q_{3}}{\sqrt{A_{2}} B_{1}^{2}}-\frac{K_{4} p Q_{3}}{A_{2}^{3 / 2} B_{1}}+\frac{2 K_{4} p Q_{3}}{\sqrt{A_{4}} B_{2}^{2}}+\frac{K_{4} p Q_{3}}{A_{4}^{3 / 2} B_{2}}+\frac{K_{2} Q_{4}}{\sqrt{A_{1}} B_{1}}-\frac{K_{2} Q_{4}}{\sqrt{A_{3}} B_{2}}+\frac{2 K_{4} p Q_{4}}{\sqrt{A_{1}} B_{1}^{2}} \\
& +\frac{K_{4} p Q_{4}}{A_{1}^{3 / 2} B_{1}}-\frac{2 K_{4} p Q_{4}}{\sqrt{A_{3}} B_{2}^{2}}-\frac{K_{4} p Q_{4}}{A_{3}^{3 / 2} B_{2}}-\frac{p^{2} Q_{4}\left(3 A_{1}-Q_{4}^{2}\right)}{A_{1}^{3 / 2} B_{1}^{2}}+\frac{p^{2} Q_{4}\left(3 A_{3}-Q_{4}^{2}\right)}{A_{3}^{3 / 2} B_{2}^{2}} \\
& +\frac{p^{2} Q_{3} S_{1}}{A_{2}^{3 / 2} B_{1}^{2}}-\frac{p^{2} Q_{3} S_{2}}{A_{4}^{3 / 2} B_{2}^{2}}+\frac{6 p^{3} Q_{3} z}{A_{2}^{3 / 2} B_{1}^{2}}-\frac{6 p^{3} Q_{3} z}{A_{4}^{3 / 2} B_{2}^{2}}-\frac{6 p^{3} Q_{4} z}{A_{1}^{3 / 2} B_{1}^{2}}+\frac{6 p^{3} Q_{4} z}{A_{3}^{3 / 2} B_{2}^{2}} \\
& -\frac{2 p Q_{4}\left(3 A_{1}-Q_{4}^{2}\right) z}{A_{1}^{3 / 2} B_{1}^{2}}+\frac{4 p^{3} Q_{4}\left(3 A_{1}-Q_{4}^{2}\right) z}{A_{1}^{3 / 2} B_{1}^{3}}+\frac{3 p^{3} Q_{4}\left(3 A_{1}-Q_{4}^{2}\right) z}{A_{1}^{5 / 2} B_{1}^{2}} \\
& +\frac{2 p Q_{4}\left(3 A_{3}-Q_{4}^{2}\right) z}{A_{3}^{3 / 2} B_{2}^{2}}-\frac{4 p^{3} Q_{4}\left(3 A_{3}-Q_{4}^{2}\right) z}{A_{3}^{3 / 2} B_{2}^{3}}-\frac{3 p^{3} Q_{4}\left(3 A_{3}-Q_{4}^{2}\right) z}{A_{3}^{5 / 2} B_{2}^{2}} \\
& +\frac{2 p Q_{3} S_{1} z}{A_{2}^{3 / 2} B_{1}^{2}}-\frac{4 p^{3} Q_{3} S_{1} z}{A_{2}^{3 / 2} B_{1}^{3}}-\frac{3 p^{3} Q_{3} S_{1} z}{A_{2}^{5 / 2} B_{1}^{2}}-\frac{2 p Q_{3} S_{2} z}{A_{4}^{3 / 2} B_{2}^{2}}+\frac{4 p^{3} Q_{3} S_{2} z}{A_{4}^{3 / 2} B_{2}^{3}} \\
& \left.+\frac{3 p^{3} Q_{3} S_{2} z}{A_{4}^{5 / 2} B_{2}^{2}}\right)-\frac{1}{4 K \pi} b_{x} c\left(K _ { 2 } \left(\frac{Q_{3}}{\sqrt{A_{2}} B_{1}}-\frac{Q_{3}}{\sqrt{A_{4}} B_{2}}-\frac{2 Q_{1}^{2} Q_{3}}{\sqrt{A_{2}} B_{1}^{2}}-\frac{Q_{1}^{2} Q_{3}}{A_{2}^{3 / 2} B_{1}}\right.\right. \\
& +\frac{2 Q_{2}^{2} Q_{3}}{\sqrt{A_{4}} B_{2}^{2}}+\frac{Q_{2}^{2} Q_{3}}{A_{4}^{3 / 2} B_{2}}-\frac{Q_{4}}{\sqrt{A_{1}} B_{1}}+\frac{Q_{4}}{\sqrt{A_{3}} B_{2}}+\frac{2 Q_{1}^{2} Q_{4}}{\sqrt{A_{1}} B_{1}^{2}}+\frac{Q_{1}^{2} Q_{4}}{A_{1}^{3 / 2} B_{1}}-\frac{2 Q_{2}^{2} Q_{4}}{\sqrt{A_{3}} B_{2}^{2}} \\
& \left.-\frac{Q_{2}^{2} Q_{4}}{A_{3}^{3 / 2} B_{2}}\right)+\frac{6 p Q_{1}^{2} Q_{3} z}{A_{2}^{3 / 2} B_{1}^{2}}-\frac{6 p Q_{2}^{2} Q_{3} z}{A_{4}^{3 / 2} B_{2}^{2}}-\frac{6 p Q_{1}^{2} Q_{4} z}{A_{1}^{3 / 2} B_{1}^{2}}+\frac{6 p Q_{2}^{2} Q_{4} z}{A_{3}^{3 / 2} B_{2}^{2}} \\
& -\frac{p Q_{4}\left(3 A_{1}-Q_{4}^{2}\right) z}{A_{1}^{3 / 2} B_{1}^{2}}+\frac{4 p Q_{1}^{2} Q_{4}\left(3 A_{1}-Q_{4}^{2}\right) z}{A_{1}^{3 / 2} B_{1}^{3}}+\frac{3 p Q_{1}^{2} Q_{4}\left(3 A_{1}-Q_{4}^{2}\right) z}{A_{1}^{5 / 2} B_{1}^{2}} \\
& +\frac{p Q_{4}\left(3 A_{3}-Q_{4}^{2}\right) z}{A_{3}^{3 / 2} B_{2}^{2}}-\frac{4 p Q_{2}^{2} Q_{4}\left(3 A_{3}-Q_{4}^{2}\right) z}{A_{3}^{3 / 2} B_{2}^{3}}-\frac{3 p Q_{2}^{2} Q_{4}\left(3 A_{3}-Q_{4}^{2}\right) z}{A_{3}^{5 / 2} B_{2}^{2}} \\
& +\frac{p Q_{3} S_{1} z}{A_{2}^{3 / 2} B_{1}^{2}}-\frac{4 p Q_{1}^{2} Q_{3} S_{1} z}{A_{2}^{3 / 2} B_{1}^{3}}-\frac{3 p Q_{1}^{2} Q_{3} S_{1} z}{A_{2}^{5 / 2} B_{1}^{2}}-\frac{p Q_{3} S_{2} z}{A_{4}^{3 / 2} B_{2}^{2}}+\frac{4 p Q_{2}^{2} Q_{3} S_{2} z}{A_{4}^{3 / 2} B_{2}^{3}}
\end{aligned}
$$




$$
\begin{aligned}
& \left.+\frac{3 p Q_{2}^{2} Q_{3} S_{2} z}{A_{4}^{5 / 2} B_{2}^{2}}\right)+\frac{b_{x} c}{-4 \pi K}\left(K_{2}\left(\frac{Q_{3}}{A_{2}^{3 / 2}}-\frac{Q_{3}}{A_{4}^{3 / 2}}-\frac{Q_{4}}{A_{1}^{3 / 2}}+\frac{Q_{4}}{A_{3}^{3 / 2}}\right)+\frac{3 p Q_{3} z}{A_{2}^{5 / 2}}\right. \\
& \left.\left.-\frac{3 p Q_{3} z}{A_{4}^{5 / 2}}-\frac{3 p Q_{4} z}{A_{1}^{5 / 2}}+\frac{3 p Q_{4} z}{A_{3}^{5 / 2}}\right)\right) \\
& \frac{\sigma_{x y}}{\mu}=\frac{b_{x} c}{4 K \pi}\left(K_{2}\left(\frac{Q_{1}}{A_{1}^{3 / 2}}-\frac{Q_{1}}{A_{2}^{3 / 2}}+\frac{Q_{2}}{A_{3}^{3 / 2}}-\frac{Q_{2}}{A_{4}^{3 / 2}}\right)+\frac{3 p Q_{1} z}{A_{1}^{5 / 2}}-\frac{3 p Q_{1} z}{A_{2}^{5 / 2}}+\frac{3 p Q_{2} z}{A_{3}^{5 / 2}}\right. \\
& \left.-\frac{3 p Q_{2} z}{A_{4}^{5 / 2}}\right)+\frac{1}{4 K \pi} b_{x} c\left(K _ { 2 } \left(\frac{Q_{1}}{\sqrt{A_{1}} B_{1}}-\frac{Q_{1}}{\sqrt{A_{2}} B_{1}}+\frac{Q_{2}}{\sqrt{A_{3}} B_{2}}-\frac{Q_{2}}{\sqrt{A_{4}} B_{2}}+\frac{Q_{1} Q_{3}^{2}}{A_{2}^{3 / 2} B_{1}}\right.\right. \\
& \left.+\frac{Q_{2} Q_{3}^{2}}{A_{4}^{3 / 2} B_{2}}-\frac{Q_{1} Q_{4}^{2}}{A_{1}^{3 / 2} B_{1}}-\frac{Q_{2} Q_{4}^{2}}{A_{3}^{3 / 2} B_{2}}\right)-\frac{4 p Q_{1} Q_{3}^{2} z}{A_{2}^{3 / 2} B_{1}^{2}}-\frac{4 p Q_{2} Q_{3}^{2} z}{A_{4}^{3 / 2} B_{2}^{2}}+\frac{4 p Q_{1} Q_{4}^{2} z}{A_{1}^{3 / 2} B_{1}^{2}} \\
& +\frac{4 p Q_{2} Q_{4}^{2} z}{A_{3}^{3 / 2} B_{2}^{2}}+\frac{p Q_{1}\left(3 A_{1}-Q_{4}^{2}\right) z}{A_{1}^{3 / 2} B_{1}^{2}}-\frac{3 p Q_{1} Q_{4}^{2}\left(3 A_{1}-Q_{4}^{2}\right) z}{A_{1}^{5 / 2} B_{1}^{2}}+\frac{p Q_{2}\left(3 A_{3}-Q_{4}^{2}\right) z}{A_{3}^{3 / 2} B_{2}^{2}} \\
& \left.-\frac{3 p Q_{2} Q_{4}^{2}\left(3 A_{3}-Q_{4}^{2}\right) z}{A_{3}^{5 / 2} B_{2}^{2}}-\frac{p Q_{1} S_{1} z}{A_{2}^{3 / 2} B_{1}^{2}}+\frac{3 p Q_{1} Q_{3}^{2} S_{1} z}{A_{2}^{5 / 2} B_{1}^{2}}-\frac{p Q_{2} S_{2} z}{A_{4}^{3 / 2} B_{2}^{2}}+\frac{3 p Q_{2} Q_{3}^{2} S_{2} z}{A_{4}^{5 / 2} B_{2}^{2}}\right) \\
& \frac{\sigma_{x z}}{\mu}=\frac{1}{4 K \pi} b_{x} c\left(\frac{2 G_{1} Q_{1} Q_{3}}{\sqrt{A_{2}} B_{1}^{2}}+\frac{G_{1} Q_{1} Q_{3}}{A_{2}^{3 / 2} B_{1}}+\frac{2 G_{1} Q_{2} Q_{3}}{\sqrt{A_{4}} B_{2}^{2}}+\frac{G_{1} Q_{2} Q_{3}}{A_{4}^{3 / 2} B_{2}}-\frac{2 G_{1} Q_{1} Q_{4}}{\sqrt{A_{1}} B_{1}^{2}}\right. \\
& -\frac{G_{1} Q_{1} Q_{4}}{A_{1}^{3 / 2} B_{1}}-\frac{2 G_{1} Q_{2} Q_{4}}{\sqrt{A_{3}} B_{2}^{2}}-\frac{G_{1} Q_{2} Q_{4}}{A_{3}^{3 / 2} B_{2}}-\frac{6 p^{2} Q_{1} Q_{3} z}{A_{2}^{3 / 2} B_{1}^{2}}-\frac{6 p^{2} Q_{2} Q_{3} z}{A_{4}^{3 / 2} B_{2}^{2}}+\frac{6 p^{2} Q_{1} Q_{4} z}{A_{1}^{3 / 2} B_{1}^{2}} \\
& +\frac{6 p^{2} Q_{2} Q_{4} z}{A_{3}^{3 / 2} B_{2}^{2}}-\frac{4 p^{2} Q_{1} Q_{4}\left(3 A_{1}-Q_{4}^{2}\right) z}{A_{1}^{3 / 2} B_{1}^{3}}-\frac{3 p^{2} Q_{1} Q_{4}\left(3 A_{1}-Q_{4}^{2}\right) z}{A_{1}^{5 / 2} B_{1}^{2}} \\
& -\frac{4 p^{2} Q_{2} Q_{4}\left(3 A_{3}-Q_{4}^{2}\right) z}{A_{3}^{3 / 2} B_{2}^{3}}-\frac{3 p^{2} Q_{2} Q_{4}\left(3 A_{3}-Q_{4}^{2}\right) z}{A_{3}^{5 / 2} B_{2}^{2}}+\frac{4 p^{2} Q_{1} Q_{3} S_{1} z}{A_{2}^{3 / 2} B_{1}^{3}} \\
& \left.+\frac{3 p^{2} Q_{1} Q_{3} S_{1} z}{A_{2}^{5 / 2} B_{1}^{2}}+\frac{4 p^{2} Q_{2} Q_{3} S_{2} z}{A_{4}^{3 / 2} B_{2}^{3}}+\frac{3 p^{2} Q_{2} Q_{3} S_{2} z}{A_{4}^{5 / 2} B_{2}^{2}}\right)+\frac{1}{4 K \pi} b_{x} c\left(K _ { 2 } \left(\frac{2 p Q_{1} Q_{3}}{\sqrt{A_{2}} B_{1}^{2}}\right.\right. \\
& \left.+\frac{p Q_{1} Q_{3}}{A_{2}^{3 / 2} B_{1}}+\frac{2 p Q_{2} Q_{3}}{\sqrt{A_{4}} B_{2}^{2}}+\frac{p Q_{2} Q_{3}}{A_{4}^{3 / 2} B_{2}}-\frac{2 p Q_{1} Q_{4}}{\sqrt{A_{1}} B_{1}^{2}}-\frac{p Q_{1} Q_{4}}{A_{1}^{3 / 2} B_{1}}-\frac{2 p Q_{2} Q_{4}}{\sqrt{A_{3}} B_{2}^{2}}-\frac{p Q_{2} Q_{4}}{A_{3}^{3 / 2} B_{2}}\right) \\
& +\frac{p Q_{1} Q_{4}\left(3 A_{1}-Q_{4}^{2}\right)}{A_{1}^{3 / 2} B_{1}^{2}}+\frac{p Q_{2} Q_{4}\left(3 A_{3}-Q_{4}^{2}\right)}{A_{3}^{3 / 2} B_{2}^{2}}-\frac{p Q_{1} Q_{3} S_{1}}{A_{2}^{3 / 2} B_{1}^{2}}-\frac{p Q_{2} Q_{3} S_{2}}{A_{4}^{3 / 2} B_{2}^{2}} \\
& -\frac{6 p^{2} Q_{1} Q_{3} z}{A_{2}^{3 / 2} B_{1}^{2}}-\frac{6 p^{2} Q_{2} Q_{3} z}{A_{4}^{3 / 2} B_{2}^{2}}+\frac{6 p^{2} Q_{1} Q_{4} z}{A_{1}^{3 / 2} B_{1}^{2}}+\frac{6 p^{2} Q_{2} Q_{4} z}{A_{3}^{3 / 2} B_{2}^{2}}+\frac{Q_{1} Q_{4}\left(3 A_{1}-Q_{4}^{2}\right) z}{A_{1}^{3 / 2} B_{1}^{2}} \\
& -\frac{4 p^{2} Q_{1} Q_{4}\left(3 A_{1}-Q_{4}^{2}\right) z}{A_{1}^{3 / 2} B_{1}^{3}}-\frac{3 p^{2} Q_{1} Q_{4}\left(3 A_{1}-Q_{4}^{2}\right) z}{A_{1}^{5 / 2} B_{1}^{2}}+\frac{Q_{2} Q_{4}\left(3 A_{3}-Q_{4}^{2}\right) z}{A_{3}^{3 / 2} B_{2}^{2}} \\
& -\frac{4 p^{2} Q_{2} Q_{4}\left(3 A_{3}-Q_{4}^{2}\right) z}{A_{3}^{3 / 2} B_{2}^{3}}-\frac{3 p^{2} Q_{2} Q_{4}\left(3 A_{3}-Q_{4}^{2}\right) z}{A_{3}^{5 / 2} B_{2}^{2}}-\frac{Q_{1} Q_{3} S_{1} z}{A_{2}^{3 / 2} B_{1}^{2}} \\
& \left.+\frac{4 p^{2} Q_{1} Q_{3} S_{1} z}{A_{2}^{3 / 2} B_{1}^{3}}+\frac{3 p^{2} Q_{1} Q_{3} S_{1} z}{A_{2}^{5 / 2} B_{1}^{2}}-\frac{Q_{2} Q_{3} S_{2} z}{A_{4}^{3 / 2} B_{2}^{2}}+\frac{4 p^{2} Q_{2} Q_{3} S_{2} z}{A_{4}^{3 / 2} B_{2}^{3}}+\frac{3 p^{2} Q_{2} Q_{3} S_{2} z}{A_{4}^{5 / 2} B_{2}^{2}}\right)
\end{aligned}
$$




$$
\begin{aligned}
\frac{\sigma_{y z}}{\mu}= & \frac{b_{x} c}{4 K \pi}\left(\frac{p}{A_{1}^{3 / 2}}-\frac{p}{A_{2}^{3 / 2}}-\frac{p}{A_{3}^{3 / 2}}+\frac{p}{A_{4}^{3 / 2}}+K_{2}\left(-\frac{p}{A_{1}^{3 / 2}}+\frac{p}{A_{2}^{3 / 2}}+\frac{p}{A_{3}^{3 / 2}}-\frac{p}{A_{4}^{3 / 2}}\right)\right. \\
& \left.+\frac{z}{A_{1}^{3 / 2}}-\frac{z}{A_{2}^{3 / 2}}-\frac{z}{A_{3}^{3 / 2}}+\frac{z}{A_{4}^{3 / 2}}-\frac{3 p^{2} z}{A_{1}^{5 / 2}}+\frac{3 p^{2} z}{A_{2}^{5 / 2}}+\frac{3 p^{2} z}{A_{3}^{5 / 2}}-\frac{3 p^{2} z}{A_{4}^{5 / 2}}\right) \\
& +\frac{1}{4 K \pi} b_{x} c\left(-\frac{G_{1}}{\sqrt{A_{1}} B_{1}}+\frac{G_{1}}{\sqrt{A_{2}} B_{1}}+\frac{G_{1}}{\sqrt{A_{3}} B_{2}}-\frac{G_{1}}{\sqrt{A_{4}} B_{2}}-\frac{G_{1} Q_{3}^{2}}{A_{2}^{3 / 2} B_{1}}+\frac{G_{1} Q_{3}^{2}}{A_{4}^{3 / 2} B_{2}}\right. \\
& +\frac{G_{1} Q_{4}^{2}}{A_{1}^{3 / 2} B_{1}}-\frac{G_{1} Q_{4}^{2}}{A_{3}^{3 / 2} B_{2}}+\frac{4 p^{2} Q_{3}^{2} z}{A_{2}^{3 / 2} B_{1}^{2}}-\frac{4 p^{2} Q_{3}^{2} z}{A_{4}^{3 / 2} B_{2}^{2}}-\frac{4 p^{2} Q_{4}^{2} z}{A_{1}^{3 / 2} B_{1}^{2}}+\frac{4 p^{2} Q_{4}^{2} z}{A_{3}^{3 / 2} B_{2}^{2}} \\
& -\frac{p^{2}\left(3 A_{1}-Q_{4}^{2}\right) z}{A_{1}^{3 / 2} B_{1}^{2}}+\frac{3 p^{2} Q_{4}^{2}\left(3 A_{1}-Q_{4}^{2}\right) z}{A_{1}^{5 / 2} B_{1}^{2}}+\frac{p^{2}\left(3 A_{3}-Q_{4}^{2}\right) z}{A_{3}^{3 / 2} B_{2}^{2}} \\
& \left.-\frac{3 p^{2} Q_{4}^{2}\left(3 A_{3}-Q_{4}^{2}\right) z}{A_{3}^{5 / 2} B_{2}^{2}}+\frac{p^{2} S_{1} z}{A_{2}^{3 / 2} B_{1}^{2}}-\frac{3 p^{2} Q_{3}^{2} S_{1} z}{A_{2}^{5 / 2} B_{1}^{2}}-\frac{p^{2} S_{2} z}{A_{4}^{3 / 2} B_{2}^{2}}+\frac{3 p^{2} Q_{3}^{2} S_{2} z}{A_{4}^{5 / 2} B_{2}^{2}}\right)
\end{aligned}
$$

\section{Appendix B}

The surface correction terms for stress considering only $b_{y}$ (the $y$-component of the Burgers vector):

$$
\begin{aligned}
& \frac{\sigma_{x x}}{\mu}=\frac{b_{y} c}{2 K \pi}\left(K_{2}\left(\frac{Q_{1}}{A_{1}^{3 / 2}}-\frac{Q_{1}}{A_{2}^{3 / 2}}+\frac{Q_{2}}{A_{3}^{3 / 2}}-\frac{Q_{2}}{A_{4}^{3 / 2}}\right)+\frac{3 p Q_{1} z}{A_{1}^{5 / 2}}-\frac{3 p Q_{1} z}{A_{2}^{5 / 2}}+\frac{3 p Q_{2} z}{A_{3}^{5 / 2}}\right. \\
& \left.-\frac{3 p Q_{2} z}{A_{4}^{5 / 2}}\right)-\frac{1}{K_{2}} 2 v\left(-\frac{1}{4 K \pi} b_{y} c\left(\frac{K_{2} Q_{1}}{\sqrt{A_{2}} C_{1}}-\frac{K_{2} Q_{1}}{\sqrt{A_{1}} C_{2}}+\frac{2 K_{4} p Q_{1}}{\sqrt{A_{2}} C_{1}^{2}}+\frac{K_{4} p Q_{1}}{A_{2}^{3 / 2} C_{1}}\right.\right. \\
& -\frac{2 K_{4} p Q_{1}}{\sqrt{A_{1}} C_{2}^{2}}-\frac{K_{4} p Q_{1}}{A_{1}^{3 / 2} C_{2}}-\frac{H_{1} p^{2} Q_{1}}{A_{2}^{3 / 2} C_{1}^{2}}+\frac{H_{3} p^{2} Q_{1}}{A_{1}^{3 / 2} C_{2}^{2}}+\frac{K_{2} Q_{2}}{\sqrt{A_{4}} C_{1}}-\frac{K_{2} Q_{2}}{\sqrt{A_{3}} C_{2}}+\frac{2 K_{4} p Q_{2}}{\sqrt{A_{4}} C_{1}^{2}} \\
& +\frac{K_{4} p Q_{2}}{A_{4}^{3 / 2} C_{1}}-\frac{2 K_{4} p Q_{2}}{\sqrt{A_{3}} C_{2}^{2}}-\frac{K_{4} p Q_{2}}{A_{3}^{3 / 2} C_{2}}-\frac{H_{2} p^{2} Q_{2}}{A_{4}^{3 / 2} C_{1}^{2}}+\frac{H_{4} p^{2} Q_{2}}{A_{3}^{3 / 2} C_{2}^{2}}-\frac{2 H_{1} p Q_{1} z}{A_{2}^{3 / 2} C_{1}^{2}} \\
& +\frac{2 H_{3} p Q_{1} z}{A_{1}^{3 / 2} C_{2}^{2}}-\frac{6 p^{3} Q_{1} z}{A_{2}^{3 / 2} C_{1}^{2}}+\frac{6 p^{3} Q_{1} z}{A_{1}^{3 / 2} C_{2}^{2}}+\frac{4 H_{1} p^{3} Q_{1} z}{A_{2}^{3 / 2} C_{1}^{3}}+\frac{3 H_{1} p^{3} Q_{1} z}{A_{2}^{5 / 2} C_{1}^{2}}-\frac{4 H_{3} p^{3} Q_{1} z}{A_{1}^{3 / 2} C_{2}^{3}} \\
& -\frac{3 H_{3} p^{3} Q_{1} z}{A_{1}^{5 / 2} C_{2}^{2}}-\frac{2 H_{2} p Q_{2} z}{A_{4}^{3 / 2} C_{1}^{2}}+\frac{2 H_{4} p Q_{2} z}{A_{3}^{3 / 2} C_{2}^{2}}-\frac{6 p^{3} Q_{2} z}{A_{4}^{3 / 2} C_{1}^{2}}+\frac{6 p^{3} Q_{2} z}{A_{3}^{3 / 2} C_{2}^{2}}+\frac{4 H_{2} p^{3} Q_{2} z}{A_{4}^{3 / 2} C_{1}^{3}} \\
& \left.+\frac{3 H_{2} p^{3} Q_{2} z}{A_{4}^{5 / 2} C_{1}^{2}}-\frac{4 H_{4} p^{3} Q_{2} z}{A_{3}^{3 / 2} C_{2}^{3}}-\frac{3 H_{4} p^{3} Q_{2} z}{A_{3}^{5 / 2} C_{2}^{2}}\right)-\frac{1}{4 K \pi} b_{y} c\left(K _ { 2 } \left(-\frac{Q_{1}}{\sqrt{A_{2}} C_{1}}\right.\right. \\
& +\frac{Q_{1}}{\sqrt{A_{1}} C_{2}}-\frac{Q_{2}}{\sqrt{A_{4}} C_{1}}+\frac{Q_{2}}{\sqrt{A_{3}} C_{2}}+\frac{2 Q_{1} Q_{3}^{2}}{\sqrt{A_{2}} C_{1}^{2}}+\frac{Q_{1} Q_{3}^{2}}{A_{2}^{3 / 2} C_{1}}+\frac{2 Q_{2} Q_{3}^{2}}{\sqrt{A_{4}} C_{1}^{2}}+\frac{Q_{2} Q_{3}^{2}}{A_{4}^{3 / 2} C_{1}} \\
& \left.-\frac{2 Q_{1} Q_{4}^{2}}{\sqrt{A_{1}} C_{2}^{2}}-\frac{Q_{1} Q_{4}^{2}}{A_{1}^{3 / 2} C_{2}}-\frac{2 Q_{2} Q_{4}^{2}}{\sqrt{A_{3}} C_{2}^{2}}-\frac{Q_{2} Q_{4}^{2}}{A_{3}^{3 / 2} C_{2}}\right)-\frac{H_{1} p Q_{1} z}{A_{2}^{3 / 2} C_{1}^{2}}+\frac{H_{3} p Q_{1} z}{A_{1}^{3 / 2} C_{2}^{2}}-\frac{H_{2} p Q_{2} z}{A_{4}^{3 / 2} C_{1}^{2}} \\
& +\frac{H_{4} p Q_{2} z}{A_{3}^{3 / 2} C_{2}^{2}}-\frac{6 p Q_{1} Q_{3}^{2} z}{A_{2}^{3 / 2} C_{1}^{2}}+\frac{4 H_{1} p Q_{1} Q_{3}^{2} z}{A_{2}^{3 / 2} C_{1}^{3}}+\frac{3 H_{1} p Q_{1} Q_{3}^{2} z}{A_{2}^{5 / 2} C_{1}^{2}}-\frac{6 p Q_{2} Q_{3}^{2} z}{A_{4}^{3 / 2} C_{1}^{2}} \\
& +\frac{4 H_{2} p Q_{2} Q_{3}^{2} z}{A_{4}^{3 / 2} C_{1}^{3}}+\frac{3 H_{2} p Q_{2} Q_{3}^{2} z}{A_{4}^{5 / 2} C_{1}^{2}}+\frac{6 p Q_{1} Q_{4}^{2} z}{A_{1}^{3 / 2} C_{2}^{2}}-\frac{4 H_{3} p Q_{1} Q_{4}^{2} z}{A_{1}^{3 / 2} C_{2}^{3}}-\frac{3 H_{3} p Q_{1} Q_{4}^{2} z}{A_{1}^{5 / 2} C_{2}^{2}}
\end{aligned}
$$




$$
\begin{aligned}
& \left.+\frac{6 p Q_{2} Q_{4}^{2} z}{A_{3}^{3 / 2} C_{2}^{2}}-\frac{4 H_{4} p Q_{2} Q_{4}^{2} z}{A_{3}^{3 / 2} C_{2}^{3}}-\frac{3 H_{4} p Q_{2} Q_{4}^{2} z}{A_{3}^{5 / 2} C_{2}^{2}}\right)+\frac{b_{y} c}{-4 \pi K}\left(K _ { 2 } \left(\frac{Q_{1}}{A_{1}^{3 / 2}}-\frac{Q_{1}}{A_{2}^{3 / 2}}\right.\right. \\
& \left.\left.\left.+\frac{Q_{2}}{A_{3}^{3 / 2}}-\frac{Q_{2}}{A_{4}^{3 / 2}}\right)+\frac{3 p Q_{1} z}{A_{1}^{5 / 2}}-\frac{3 p Q_{1} z}{A_{2}^{5 / 2}}+\frac{3 p Q_{2} z}{A_{3}^{5 / 2}}-\frac{3 p Q_{2} z}{A_{4}^{5 / 2}}\right)\right) \\
& \frac{\sigma_{y y}}{\mu}=\frac{1}{2 K \pi} b_{y} c\left(K _ { 2 } \left(-\frac{Q_{1}}{\sqrt{A_{2}} C_{1}}+\frac{Q_{1}}{\sqrt{A_{1}} C_{2}}-\frac{Q_{2}}{\sqrt{A_{4}} C_{1}}+\frac{Q_{2}}{\sqrt{A_{3}} C_{2}}+\frac{2 Q_{1} Q_{3}^{2}}{\sqrt{A_{2}} C_{1}^{2}}\right.\right. \\
& \left.+\frac{Q_{1} Q_{3}^{2}}{A_{2}^{3 / 2} C_{1}}+\frac{2 Q_{2} Q_{3}^{2}}{\sqrt{A_{4}} C_{1}^{2}}+\frac{Q_{2} Q_{3}^{2}}{A_{4}^{3 / 2} C_{1}}-\frac{2 Q_{1} Q_{4}^{2}}{\sqrt{A_{1}} C_{2}^{2}}-\frac{Q_{1} Q_{4}^{2}}{A_{1}^{3 / 2} C_{2}}-\frac{2 Q_{2} Q_{4}^{2}}{\sqrt{A_{3}} C_{2}^{2}}-\frac{Q_{2} Q_{4}^{2}}{A_{3}^{3 / 2} C_{2}}\right) \\
& -\frac{6 p Q_{1} Q_{3}^{2} z}{A_{2}^{3 / 2} C_{1}^{2}}-\frac{6 p Q_{2} Q_{3}^{2} z}{A_{4}^{3 / 2} C_{1}^{2}}+\frac{6 p Q_{1} Q_{4}^{2} z}{A_{1}^{3 / 2} C_{2}^{2}}+\frac{6 p Q_{2} Q_{4}^{2} z}{A_{3}^{3 / 2} C_{2}^{2}}+\frac{p Q_{1} S_{5} z}{A_{1}^{3 / 2} C_{2}^{2}}-\frac{4 p Q_{1} Q_{4}^{2} S_{5} z}{A_{1}^{3 / 2} C_{2}^{3}} \\
& -\frac{3 p Q_{1} Q_{4}^{2} S_{5} z}{A_{1}^{5 / 2} C_{2}^{2}}-\frac{p Q_{1} S_{6} z}{A_{2}^{3 / 2} C_{1}^{2}}+\frac{4 p Q_{1} Q_{3}^{2} S_{6} z}{A_{2}^{3 / 2} C_{1}^{3}}+\frac{3 p Q_{1} Q_{3}^{2} S_{6} z}{A_{2}^{5 / 2} C_{1}^{2}}+\frac{p Q_{2} S_{7} z}{A_{3}^{3 / 2} C_{2}^{2}} \\
& \left.-\frac{4 p Q_{2} Q_{4}^{2} S_{7} z}{A_{3}^{3 / 2} C_{2}^{3}}-\frac{3 p Q_{2} Q_{4}^{2} S_{7} z}{A_{3}^{5 / 2} C_{2}^{2}}-\frac{p Q_{2} S_{8} z}{A_{4}^{3 / 2} C_{1}^{2}}+\frac{4 p Q_{2} Q_{3}^{2} S_{8} z}{A_{4}^{3 / 2} C_{1}^{3}}+\frac{3 p Q_{2} Q_{3}^{2} S_{8} z}{A_{4}^{5 / 2} C_{1}^{2}}\right) \\
& -\frac{1}{K_{2}} 2 v\left(-\frac{1}{4 K \pi} b_{y} c\left(\frac{K_{2} Q_{1}}{\sqrt{A_{2}} C_{1}}-\frac{K_{2} Q_{1}}{\sqrt{A_{1}} C_{2}}+\frac{2 K_{4} p Q_{1}}{\sqrt{A_{2}} C_{1}^{2}}+\frac{K_{4} p Q_{1}}{A_{2}^{3 / 2} C_{1}}-\frac{2 K_{4} p Q_{1}}{\sqrt{A_{1}} C_{2}^{2}}\right.\right. \\
& -\frac{K_{4} p Q_{1}}{A_{1}^{3 / 2} C_{2}}-\frac{H_{1} p^{2} Q_{1}}{A_{2}^{3 / 2} C_{1}^{2}}+\frac{H_{3} p^{2} Q_{1}}{A_{1}^{3 / 2} C_{2}^{2}}+\frac{K_{2} Q_{2}}{\sqrt{A_{4}} C_{1}}-\frac{K_{2} Q_{2}}{\sqrt{A_{3}} C_{2}}+\frac{2 K_{4} p Q_{2}}{\sqrt{A_{4}} C_{1}^{2}}+\frac{K_{4} p Q_{2}}{A_{4}^{3 / 2} C_{1}} \\
& -\frac{2 K_{4} p Q_{2}}{\sqrt{A_{3}} C_{2}^{2}}-\frac{K_{4} p Q_{2}}{A_{3}^{3 / 2} C_{2}}-\frac{H_{2} p^{2} Q_{2}}{A_{4}^{3 / 2} C_{1}^{2}}+\frac{H_{4} p^{2} Q_{2}}{A_{3}^{3 / 2} C_{2}^{2}}-\frac{2 H_{1} p Q_{1} z}{A_{2}^{3 / 2} C_{1}^{2}}+\frac{2 H_{3} p Q_{1} z}{A_{1}^{3 / 2} C_{2}^{2}} \\
& -\frac{6 p^{3} Q_{1} z}{A_{2}^{3 / 2} C_{1}^{2}}+\frac{6 p^{3} Q_{1} z}{A_{1}^{3 / 2} C_{2}^{2}}+\frac{4 H_{1} p^{3} Q_{1} z}{A_{2}^{3 / 2} C_{1}^{3}}+\frac{3 H_{1} p^{3} Q_{1} z}{A_{2}^{5 / 2} C_{1}^{2}}-\frac{4 H_{3} p^{3} Q_{1} z}{A_{1}^{3 / 2} C_{2}^{3}}-\frac{3 H_{3} p^{3} Q_{1} z}{A_{1}^{5 / 2} C_{2}^{2}} \\
& -\frac{2 H_{2} p Q_{2} z}{A_{4}^{3 / 2} C_{1}^{2}}+\frac{2 H_{4} p Q_{2} z}{A_{3}^{3 / 2} C_{2}^{2}}-\frac{6 p^{3} Q_{2} z}{A_{4}^{3 / 2} C_{1}^{2}}+\frac{6 p^{3} Q_{2} z}{A_{3}^{3 / 2} C_{2}^{2}}+\frac{4 H_{2} p^{3} Q_{2} z}{A_{4}^{3 / 2} C_{1}^{3}}+\frac{3 H_{2} p^{3} Q_{2} z}{A_{4}^{5 / 2} C_{1}^{2}} \\
& \left.-\frac{4 H_{4} p^{3} Q_{2} z}{A_{3}^{3 / 2} C_{2}^{3}}-\frac{3 H_{4} p^{3} Q_{2} z}{A_{3}^{5 / 2} C_{2}^{2}}\right)-\frac{1}{4 K \pi} b_{y} c\left(K _ { 2 } \left(-\frac{Q_{1}}{\sqrt{A_{2}} C_{1}}+\frac{Q_{1}}{\sqrt{A_{1}} C_{2}}-\frac{Q_{2}}{\sqrt{A_{4}} C_{1}}\right.\right. \\
& +\frac{Q_{2}}{\sqrt{A_{3}} C_{2}}+\frac{2 Q_{1} Q_{3}^{2}}{\sqrt{A_{2}} C_{1}^{2}}+\frac{Q_{1} Q_{3}^{2}}{A_{2}^{3 / 2} C_{1}}+\frac{2 Q_{2} Q_{3}^{2}}{\sqrt{A_{4}} C_{1}^{2}}+\frac{Q_{2} Q_{3}^{2}}{A_{4}^{3 / 2} C_{1}}-\frac{2 Q_{1} Q_{4}^{2}}{\sqrt{A_{1}} C_{2}^{2}}-\frac{Q_{1} Q_{4}^{2}}{A_{1}^{3 / 2} C_{2}} \\
& \left.-\frac{2 Q_{2} Q_{4}^{2}}{\sqrt{A_{3}} C_{1}^{2}}-\frac{Q_{2} Q_{4}^{2}}{A_{3}^{3 / 2} C_{2}}\right)-\frac{H_{1} p Q_{1} z}{A_{2}^{3 / 2} C_{1}^{2}}+\frac{H_{3} p Q_{1} z}{A_{1}^{3 / 2} C_{2}^{2}}-\frac{H_{2} p Q_{2} z}{A_{4}^{3 / 2} C_{1}^{2}}+\frac{H_{4} p Q_{2} z}{A_{3}^{3 / 2} C_{2}^{2}} \\
& -\frac{6 p Q_{1} Q_{3}^{2} z}{A_{3}^{3 / 2} C_{1}^{2}}+\frac{4 H_{1} p Q_{1} Q_{3}^{2} z}{A_{2}^{3 / 2} C_{1}^{3}}+\frac{3 H_{1} p Q_{1} Q_{3}^{2} z}{A_{2}^{5 / 2} C_{1}^{2}}-\frac{6 p Q_{2} Q_{3}^{2} z}{A_{4}^{3 / 2} C_{1}^{2}}+\frac{4 H_{2} p Q_{2} Q_{3}^{2} z}{A_{4}^{3 / 2} C_{1}^{3}} \\
& +\frac{3 H_{2} p Q_{2} Q_{3}^{2} z}{A_{4}^{5 / 2} C_{1}^{2}}+\frac{6 p Q_{1} Q_{4}^{2} z}{A_{1}^{3 / 2} C_{2}^{2}}-\frac{4 H_{3} p Q_{1} Q_{4}^{2} z}{A_{1}^{3 / 2} C_{2}^{3}}-\frac{3 H_{3} p Q_{1} Q_{4}^{2} z}{A_{1}^{5 / 2} C_{2}^{2}}+\frac{6 p Q_{2} Q_{4}^{2} z}{A_{3}^{3 / 2} C_{2}^{2}} \\
& \left.-\frac{4 H_{4} p Q_{2} Q_{4}^{2} z}{A_{3}^{3 / 2} C_{2}^{3}}-\frac{3 H_{4} p Q_{2} Q_{4}^{2} z}{A_{3}^{5 / 2} C_{2}^{2}}\right)+\frac{b_{y} c}{-4 \pi K}\left(K_{2}\left(\frac{Q_{1}}{A_{1}^{3 / 2}}-\frac{Q_{1}}{A_{2}^{3 / 2}}+\frac{Q_{2}}{A_{3}^{3 / 2}}-\frac{Q_{2}}{A_{4}^{3 / 2}}\right)\right. \\
& \left.\left.+\frac{3 p Q_{1} z}{A_{1}^{5 / 2}}-\frac{3 p Q_{1} z}{A_{2}^{5 / 2}}+\frac{3 p Q_{2} z}{A_{3}^{5 / 2}}-\frac{3 p Q_{2} z}{A_{4}^{5 / 2}}\right)\right)
\end{aligned}
$$




$$
\begin{aligned}
& \frac{\sigma_{z z}}{\mu}=\frac{1}{2 K \pi} b_{y} c\left(\frac{K_{2} Q_{1}}{\sqrt{A_{2}} C_{1}}-\frac{K_{2} Q_{1}}{\sqrt{A_{1}} C_{2}}+\frac{2 G_{1} p Q_{1}}{\sqrt{A_{2}} C_{1}^{2}}+\frac{G_{1} p Q_{1}}{A_{2}^{3 / 2} C_{1}}-\frac{2 G_{1} p Q_{1}}{\sqrt{A_{1}} C_{2}^{2}}-\frac{G_{1} p Q_{1}}{A_{1}^{3 / 2} C_{2}}\right. \\
& +\frac{K_{2} Q_{2}}{\sqrt{A_{4}} C_{1}}-\frac{K_{2} Q_{2}}{\sqrt{A_{3}} C_{2}}+\frac{2 G_{1} p Q_{2}}{\sqrt{A_{4}} C_{1}^{2}}+\frac{G_{1} p Q_{2}}{A_{4}^{3 / 2} C_{1}}-\frac{2 G_{1} p Q_{2}}{\sqrt{A_{3}} C_{2}^{2}}-\frac{G_{1} p Q_{2}}{A_{3}^{3 / 2} C_{2}}+\frac{p^{2} Q_{1} S_{5}}{A_{1}^{3 / 2} C_{2}^{2}} \\
& -\frac{p^{2} Q_{1} S_{6}}{A_{2}^{3 / 2} C_{1}^{2}}+\frac{p^{2} Q_{2} S_{7}}{A_{3}^{3 / 2} C_{2}^{2}}-\frac{p^{2} Q_{2} S_{8}}{A_{4}^{3 / 2} C_{1}^{2}}-\frac{6 p^{3} Q_{1} z}{A_{2}^{3 / 2} C_{1}^{2}}+\frac{6 p^{3} Q_{1} z}{A_{1}^{3 / 2} C_{2}^{2}}-\frac{6 p^{3} Q_{2} z}{A_{4}^{3 / 2} C_{1}^{2}}+\frac{6 p^{3} Q_{2} z}{A_{3}^{3 / 2} C_{2}^{2}} \\
& +\frac{2 p Q_{1} S_{5} z}{A_{1}^{3 / 2} C_{2}^{2}}-\frac{4 p^{3} Q_{1} S_{5} z}{A_{1}^{3 / 2} C_{2}^{3}}-\frac{3 p^{3} Q_{1} S_{5} z}{A_{1}^{5 / 2} C_{2}^{2}}-\frac{2 p Q_{1} S_{6} z}{A_{3}^{3 / 2} C_{1}^{2}}+\frac{4 p^{3} Q_{1} S_{6} z}{A_{2}^{3 / 2} C_{1}^{3}}+\frac{3 p^{3} Q_{1} S_{6} z}{A_{2}^{5 / 2} C_{1}^{2}} \\
& \left.+\frac{2 p Q_{2} S_{7} z}{A_{3}^{3 / 2} C_{2}^{2}}-\frac{4 p^{3} Q_{2} S_{7} z}{A_{3}^{3 / 2} C_{2}^{3}}-\frac{3 p^{3} Q_{2} S_{7} z}{A_{3}^{5 / 2} C_{2}^{2}}-\frac{2 p Q_{2} S_{8} z}{A_{4}^{3 / 2} C_{1}^{2}}+\frac{4 p^{3} Q_{2} S_{8} z}{A_{4}^{3 / 2} C_{1}^{3}}+\frac{3 p^{3} Q_{2} S_{8} z}{A_{4}^{5 / 2} C_{1}^{2}}\right) \\
& -\frac{1}{K_{2}} 2 v\left(-\frac{1}{4 K \pi} b_{y} c\left(\frac{K_{2} Q_{1}}{\sqrt{A_{2}} C_{1}}-\frac{K_{2} Q_{1}}{\sqrt{A_{1}} C_{2}}+\frac{2 K_{4} p Q_{1}}{\sqrt{A_{2}} C_{1}^{2}}+\frac{K_{4} p Q_{1}}{A_{2}^{3 / 2} C_{1}}-\frac{2 K_{4} p Q_{1}}{\sqrt{A_{1}} C_{2}^{2}}\right.\right. \\
& -\frac{K_{4} p Q_{1}}{A_{1}^{3 / 2} C_{2}}-\frac{H_{1} p^{2} Q_{1}}{A_{2}^{3 / 2} C_{1}^{2}}+\frac{H_{3} p^{2} Q_{1}}{A_{1}^{3 / 2} C_{2}^{2}}+\frac{K_{2} Q_{2}}{\sqrt{A_{4}} C_{1}}-\frac{K_{2} Q_{2}}{\sqrt{A_{3}} C_{2}}+\frac{2 K_{4} p Q_{2}}{\sqrt{A_{4}} C_{1}^{2}}+\frac{K_{4} p Q_{2}}{A_{4}^{3 / 2} C_{1}} \\
& -\frac{2 K_{4} p Q_{2}}{\sqrt{A_{3}} C_{2}^{2}}-\frac{K_{4} p Q_{2}}{A_{3}^{3 / 2} C_{2}}-\frac{H_{2} p^{2} Q_{2}}{A_{4}^{3 / 2} C_{1}^{2}}+\frac{H_{4} p^{2} Q_{2}}{A_{3}^{3 / 2} C_{2}^{2}}-\frac{2 H_{1} p Q_{1} z}{A_{2}^{3 / 2} C_{1}^{2}}+\frac{2 H_{3} p Q_{1} z}{A_{1}^{3 / 2} C_{2}^{2}} \\
& -\frac{6 p^{3} Q_{1} z}{A_{2}^{3 / 2} C_{1}^{2}}+\frac{6 p^{3} Q_{1} z}{A_{1}^{3 / 2} C_{2}^{2}}+\frac{4 H_{1} p^{3} Q_{1} z}{A_{2}^{3 / 2} C_{1}^{3}}+\frac{3 H_{1} p^{3} Q_{1} z}{A_{2}^{5 / 2} C_{1}^{2}}-\frac{4 H_{3} p^{3} Q_{1} z}{A_{1}^{3 / 2} C_{2}^{3}}-\frac{3 H_{3} p^{3} Q_{1} z}{A_{1}^{5 / 2} C_{2}^{2}} \\
& -\frac{2 H_{2} p Q_{2} z}{A_{4}^{3 / 2} C_{1}^{2}}+\frac{2 H_{4} p Q_{2} z}{A_{3}^{3 / 2} C_{2}^{2}}-\frac{6 p^{3} Q_{2} z}{A_{4}^{3 / 2} C_{1}^{2}}+\frac{6 p^{3} Q_{2} z}{A_{3}^{3 / 2} C_{2}^{2}}+\frac{4 H_{2} p^{3} Q_{2} z}{A_{4}^{3 / 2} C_{1}^{3}}+\frac{3 H_{2} p^{3} Q_{2} z}{A_{4}^{5 / 2} C_{1}^{2}} \\
& \left.-\frac{4 H_{4} p^{3} Q_{2} z}{A_{3}^{3 / 2} C_{2}^{3}}-\frac{3 H_{4} p^{3} Q_{2} z}{A_{3}^{5 / 2} C_{2}^{2}}\right)-\frac{1}{4 K \pi} b_{y} c\left(K _ { 2 } \left(-\frac{Q_{1}}{\sqrt{A_{2}} C_{1}}+\frac{Q_{1}}{\sqrt{A_{1}} C_{2}}-\frac{Q_{2}}{\sqrt{A_{4}} C_{1}}\right.\right. \\
& +\frac{Q_{2}}{\sqrt{A_{3}} C_{2}}+\frac{2 Q_{1} Q_{3}^{2}}{\sqrt{A_{2}} C_{1}^{2}}+\frac{Q_{1} Q_{3}^{2}}{A_{2}^{3 / 2} C_{1}}+\frac{2 Q_{2} Q_{3}^{2}}{\sqrt{A_{4}} C_{1}^{2}}+\frac{Q_{2} Q_{3}^{2}}{A_{4}^{3 / 2} C_{1}}-\frac{2 Q_{1} Q_{4}^{2}}{\sqrt{A_{1}} C_{2}^{2}}-\frac{Q_{1} Q_{4}^{2}}{A_{1}^{3 / 2} C_{2}} \\
& \left.-\frac{2 Q_{2} Q_{4}^{2}}{\sqrt{A_{3}} C_{2}^{2}}-\frac{Q_{2} Q_{4}^{2}}{A_{3}^{3 / 2} C_{2}}\right)-\frac{H_{1} p Q_{1} z}{A_{2}^{3 / 2} C_{1}^{2}}+\frac{H_{3} p Q_{1} z}{A_{1}^{3 / 2} C_{2}^{2}}-\frac{H_{2} p Q_{2} z}{A_{4}^{3 / 2} C_{1}^{2}}+\frac{H_{4} p Q_{2} z}{A_{3}^{3 / 2} C_{2}^{2}} \\
& -\frac{6 p Q_{1} Q_{3}^{2} z}{A_{2}^{3 / 2} C_{1}^{2}}+\frac{4 H_{1} p Q_{1} Q_{3}^{2} z}{A_{2}^{3 / 2} C_{1}^{3}}+\frac{3 H_{1} p Q_{1} Q_{3}^{2} z}{A_{2}^{5 / 2} C_{1}^{2}}-\frac{6 p Q_{2} Q_{3}^{2} z}{A_{4}^{3 / 2} C_{1}^{2}}+\frac{4 H_{2} p Q_{2} Q_{3}^{2} z}{A_{4}^{3 / 2} C_{1}^{3}} \\
& +\frac{3 H_{2} p Q_{2} Q_{3}^{2} z}{A_{4}^{5 / 2} C_{1}^{2}}+\frac{6 p Q_{1} Q_{4}^{2} z}{A_{1}^{3 / 2} C_{2}^{2}}-\frac{4 H_{3} p Q_{1} Q_{4}^{2} z}{A_{1}^{3 / 2} C_{2}^{3}}-\frac{3 H_{3} p Q_{1} Q_{4}^{2} z}{A_{1}^{5 / 2} C_{2}^{2}}+\frac{6 p Q_{2} Q_{4}^{2} z}{A_{3}^{3 / 2} C_{2}^{2}} \\
& \left.-\frac{4 H_{4} p Q_{2} Q_{4}^{2} z}{A_{3}^{3 / 2} C_{2}^{3}}-\frac{3 H_{4} p Q_{2} Q_{4}^{2} z}{A_{3}^{5 / 2} C_{2}^{2}}\right)+\frac{b_{y} c}{-4 \pi K}\left(K_{2}\left(\frac{Q_{1}}{A_{1}^{3 / 2}}-\frac{Q_{1}}{A_{2}^{3 / 2}}+\frac{Q_{2}}{A_{3}^{3 / 2}}-\frac{Q_{2}}{A_{4}^{3 / 2}}\right)\right. \\
& \left.\left.+\frac{3 p Q_{1} z}{A_{1}^{5 / 2}}-\frac{3 p Q_{1} z}{A_{2}^{5 / 2}}+\frac{3 p Q_{2} z}{A_{3}^{5 / 2}}-\frac{3 p Q_{2} z}{A_{4}^{5 / 2}}\right)\right) \\
& \frac{\sigma_{x y}}{\mu}=\frac{b_{y} c}{4 K \pi}\left(K_{2}\left(\frac{Q_{3}}{A_{2}^{3 / 2}}-\frac{Q_{3}}{A_{4}^{3 / 2}}-\frac{Q_{4}}{A_{1}^{3 / 2}}+\frac{Q_{4}}{A_{3}^{3 / 2}}\right)+\frac{3 p Q_{3} z}{A_{2}^{5 / 2}}-\frac{3 p Q_{3} z}{A_{4}^{5 / 2}}-\frac{3 p Q_{4} z}{A_{1}^{5 / 2}}\right. \\
& \left.+\frac{3 p Q_{4} z}{A_{3}^{5 / 2}}\right)+\frac{1}{4 K \pi} b_{y} c\left(K _ { 2 } \left(\frac{Q_{3}}{\sqrt{A_{2}} C_{1}}-\frac{Q_{3}}{\sqrt{A_{4}} C_{1}}-\frac{Q_{1}^{2} Q_{3}}{A_{2}^{3 / 2} C_{1}}+\frac{Q_{2}^{2} Q_{3}}{A_{4}^{3 / 2} C_{1}}\right.\right.
\end{aligned}
$$




$$
\begin{aligned}
& \left.-\frac{Q_{4}}{\sqrt{A_{1}} C_{2}}+\frac{Q_{4}}{\sqrt{A_{3}} C_{2}}+\frac{Q_{1}^{2} Q_{4}}{A_{1}^{3 / 2} C_{2}}-\frac{Q_{2}^{2} Q_{4}}{A_{3}^{3 / 2} C_{2}}\right)+\frac{4 p Q_{1}^{2} Q_{3} z}{A_{2}^{3 / 2} C_{1}^{2}}-\frac{4 p Q_{2}^{2} Q_{3} z}{A_{4}^{3 / 2} C_{1}^{2}} \\
& -\frac{4 p Q_{1}^{2} Q_{4} z}{A_{1}^{3 / 2} C_{2}^{2}}+\frac{4 p Q_{2}^{2} Q_{4} z}{A_{3}^{3 / 2} C_{2}^{2}}-\frac{p Q_{4} S_{5} z}{A_{1}^{3 / 2} C_{2}^{2}}+\frac{3 p Q_{1}^{2} Q_{4} S_{5} z}{A_{1}^{5 / 2} C_{2}^{2}}+\frac{p Q_{3} S_{6} z}{A_{2}^{3 / 2} C_{1}^{2}} \\
& \left.-\frac{3 p Q_{1}^{2} Q_{3} S_{6} z}{A_{2}^{5 / 2} C_{1}^{2}}+\frac{p Q_{4} S_{7} z}{A_{3}^{3 / 2} C_{2}^{2}}-\frac{3 p Q_{2}^{2} Q_{4} S_{7} z}{A_{3}^{5 / 2} C_{2}^{2}}-\frac{p Q_{3} S_{8} z}{A_{4}^{3 / 2} C_{1}^{2}}+\frac{3 p Q_{2}^{2} Q_{3} S_{8} z}{A_{4}^{5 / 2} C_{1}^{2}}\right) \\
& \frac{\sigma_{x z}}{\mu}=\frac{b_{y} c}{4 K \pi}\left(\frac{p}{A_{1}^{3 / 2}}-\frac{p}{A_{2}^{3 / 2}}-\frac{p}{A_{3}^{3 / 2}}+\frac{p}{A_{4}^{3 / 2}}+K_{2}\left(-\frac{p}{A_{1}^{3 / 2}}+\frac{p}{A_{2}^{3 / 2}}+\frac{p}{A_{3}^{3 / 2}}-\frac{p}{A_{4}^{3 / 2}}\right)\right. \\
& \left.+\frac{z}{A_{1}^{3 / 2}}-\frac{z}{A_{2}^{3 / 2}}-\frac{z}{A_{3}^{3 / 2}}+\frac{z}{A_{4}^{3 / 2}}-\frac{3 p^{2} z}{A_{1}^{5 / 2}}+\frac{3 p^{2} z}{A_{2}^{5 / 2}}+\frac{3 p^{2} z}{A_{3}^{5 / 2}}-\frac{3 p^{2} z}{A_{4}^{5 / 2}}\right) \\
& +\frac{1}{4 K \pi} b_{y} c\left(\frac{G_{1}}{\sqrt{A_{2}} C_{1}}-\frac{G_{1}}{\sqrt{A_{4}} C_{1}}-\frac{G_{1}}{\sqrt{A_{1}} C_{2}}+\frac{G_{1}}{\sqrt{A_{3}} C_{2}}-\frac{G_{1} Q_{1}^{2}}{A_{2}^{3 / 2} C_{1}}\right. \\
& +\frac{G_{1} Q_{1}^{2}}{A_{1}^{3 / 2} C_{2}}+\frac{G_{1} Q_{2}^{2}}{A_{4}^{3 / 2} C_{1}}-\frac{G_{1} Q_{2}^{2}}{A_{3}^{3 / 2} C_{2}}+\frac{4 p^{2} Q_{1}^{2} z}{A_{2}^{3 / 2} C_{1}^{2}}-\frac{4 p^{2} Q_{1}^{2} z}{A_{1}^{3 / 2} C_{2}^{2}}-\frac{4 p^{2} Q_{2}^{2} z}{A_{4}^{3 / 2} C_{1}^{2}} \\
& +\frac{4 p^{2} Q_{2}^{2} z}{A_{3}^{3 / 2} C_{2}^{2}}-\frac{p^{2} S_{5} z}{A_{1}^{3 / 2} C_{2}^{2}}+\frac{3 p^{2} Q_{1}^{2} S_{5} z}{A_{1}^{5 / 2} C_{2}^{2}}+\frac{p^{2} S_{6} z}{A_{2}^{3 / 2} C_{1}^{2}}-\frac{3 p^{2} Q_{1}^{2} S_{6} z}{A_{2}^{5 / 2} C_{1}^{2}} \\
& \left.+\frac{p^{2} S_{7} z}{A_{3}^{3 / 2} C_{2}^{2}}-\frac{3 p^{2} Q_{2}^{2} S_{7} z}{A_{3}^{5 / 2} C_{2}^{2}}-\frac{p^{2} S_{8} z}{A_{4}^{3 / 2} C_{1}^{2}}+\frac{3 p^{2} Q_{2}^{2} S_{8} z}{A_{4}^{5 / 2} C_{1}^{2}}\right) \\
& \frac{\sigma_{y z}}{\mu}=\frac{1}{4 K \pi} b_{y} c\left(\frac{2 G_{1} Q_{1} Q_{3}}{\sqrt{A_{2}} C_{1}^{2}}+\frac{G_{1} Q_{1} Q_{3}}{A_{2}^{3 / 2} C_{1}}+\frac{2 G_{1} Q_{2} Q_{3}}{\sqrt{A_{4}} C_{1}^{2}}+\frac{G_{1} Q_{2} Q_{3}}{A_{4}^{3 / 2} C_{1}}-\frac{2 G_{1} Q_{1} Q_{4}}{\sqrt{A_{1}} C_{2}^{2}}\right. \\
& -\frac{G_{1} Q_{1} Q_{4}}{A_{1}^{3 / 2} C_{2}}-\frac{2 G_{1} Q_{2} Q_{4}}{\sqrt{A_{3}} C_{2}^{2}}-\frac{G_{1} Q_{2} Q_{4}}{A_{3}^{3 / 2} C_{2}}-\frac{6 p^{2} Q_{1} Q_{3} z}{A_{2}^{3 / 2} C_{1}^{2}}-\frac{6 p^{2} Q_{2} Q_{3} z}{A_{4}^{3 / 2} C_{1}^{2}}+\frac{6 p^{2} Q_{1} Q_{4} z}{A_{1}^{3 / 2} C_{2}^{2}} \\
& +\frac{6 p^{2} Q_{2} Q_{4} z}{A_{3}^{3 / 2} C_{2}^{2}}-\frac{4 p^{2} Q_{1} Q_{4} S_{5} z}{A_{1}^{3 / 2} C_{2}^{3}}-\frac{3 p^{2} Q_{1} Q_{4} S_{5} z}{A_{1}^{5 / 2} C_{2}^{2}}+\frac{4 p^{2} Q_{1} Q_{3} S_{6} z}{A_{2}^{3 / 2} C_{1}^{3}}+\frac{3 p^{2} Q_{1} Q_{3} S_{6} z}{A_{2}^{5 / 2} C_{1}^{2}} \\
& \left.-\frac{4 p^{2} Q_{2} Q_{4} S_{7} z}{A_{3}^{3 / 2} C_{2}^{3}}-\frac{3 p^{2} Q_{2} Q_{4} S_{7} z}{A_{3}^{5 / 2} C_{2}^{2}}+\frac{4 p^{2} Q_{2} Q_{3} S_{8} z}{A_{4}^{3 / 2} C_{1}^{3}}+\frac{3 p^{2} Q_{2} Q_{3} S_{8} z}{A_{4}^{5 / 2} C_{1}^{2}}\right) \\
& +\frac{1}{4 K \pi} b_{y} c\left(K _ { 2 } \left(\frac{2 p Q_{1} Q_{3}}{\sqrt{A_{2}} C_{1}^{2}}+\frac{p Q_{1} Q_{3}}{A_{2}^{3 / 2} C_{1}}+\frac{2 p Q_{2} Q_{3}}{\sqrt{A_{4}} C_{1}^{2}}+\frac{p Q_{2} Q_{3}}{A_{4}^{3 / 2} C_{1}}-\frac{2 p Q_{1} Q_{4}}{\sqrt{A_{1}} C_{2}^{2}}\right.\right. \\
& \left.-\frac{p Q_{1} Q_{4}}{A_{1}^{3 / 2} C_{2}}-\frac{2 p Q_{2} Q_{4}}{\sqrt{A_{3}} C_{2}^{2}}-\frac{p Q_{2} Q_{4}}{A_{3}^{3 / 2} C_{2}}\right)+\frac{p Q_{1} Q_{4} S_{5}}{A_{1}^{3 / 2} C_{2}^{2}}-\frac{p Q_{1} Q_{3} S_{6}}{A_{2}^{3 / 2} C_{1}^{2}}+\frac{p Q_{2} Q_{4} S_{7}}{A_{3}^{3 / 2} C_{2}^{2}} \\
& -\frac{p Q_{2} Q_{3} S_{8}}{A_{4}^{3 / 2} C_{1}^{2}}-\frac{6 p^{2} Q_{1} Q_{3} z}{A_{2}^{3 / 2} C_{1}^{2}}-\frac{6 p^{2} Q_{2} Q_{3} z}{A_{4}^{3 / 2} C_{1}^{2}}+\frac{6 p^{2} Q_{1} Q_{4} z}{A_{1}^{3 / 2} C_{2}^{2}}+\frac{6 p^{2} Q_{2} Q_{4} z}{A_{3}^{3 / 2} C_{2}^{2}} \\
& +\frac{Q_{1} Q_{4} S_{5} z}{A_{1}^{3 / 2} C_{2}^{2}}-\frac{4 p^{2} Q_{1} Q_{4} S_{5} z}{A_{1}^{3 / 2} C_{2}^{3}}-\frac{3 p^{2} Q_{1} Q_{4} S_{5} z}{A_{1}^{5 / 2} C_{2}^{2}}-\frac{Q_{1} Q_{3} S_{6} z}{A_{2}^{3 / 2} C_{1}^{2}}+\frac{4 p^{2} Q_{1} Q_{3} S_{6} z}{A_{2}^{3 / 2} C_{1}^{3}} \\
& +\frac{3 p^{2} Q_{1} Q_{3} S_{6} z}{A_{2}^{5 / 2} C_{1}^{2}}+\frac{Q_{2} Q_{4} S_{7} z}{A_{3}^{3 / 2} C_{2}^{2}}-\frac{4 p^{2} Q_{2} Q_{4} S_{7} z}{A_{3}^{3 / 2} C_{2}^{3}}-\frac{3 p^{2} Q_{2} Q_{4} S_{7} z}{A_{3}^{5 / 2} C_{2}^{2}}-\frac{Q_{2} Q_{3} S_{8} z}{A_{4}^{3 / 2} C_{1}^{2}} \\
& \left.+\frac{4 p^{2} Q_{2} Q_{3} S_{8} z}{A_{4}^{3 / 2} C_{1}^{3}}+\frac{3 p^{2} Q_{2} Q_{3} S_{8} z}{A_{4}^{5 / 2} C_{1}^{2}}\right)
\end{aligned}
$$




\section{Appendix C}

The surface correction terms for stress considering only $b_{z}$ (the $z$-component of the Burgers vector):

$$
\begin{aligned}
& \frac{\sigma_{x x}}{\mu}=\frac{1}{2 K \pi} b_{z} c\left(G _ { 2 } \left(\frac{2 Q_{1} Q_{3}}{\sqrt{A_{2}} B_{1}^{2}}+\frac{Q_{1} Q_{3}}{A_{2}^{3 / 2} B_{1}}+\frac{2 Q_{2} Q_{3}}{\sqrt{A_{4}} B_{2}^{2}}+\frac{Q_{2} Q_{3}}{A_{4}^{3 / 2} B_{2}}-\frac{2 Q_{1} Q_{4}}{\sqrt{A_{1}} B_{1}^{2}}-\frac{Q_{1} Q_{4}}{A_{1}^{3 / 2} B_{1}}\right.\right. \\
& \left.-\frac{2 Q_{2} Q_{4}}{\sqrt{A_{3}} B_{2}^{2}}-\frac{Q_{2} Q_{4}}{A_{3}^{3 / 2} B_{2}}\right)+\frac{6 p^{2} Q_{1} Q_{3} z}{A_{2}^{3 / 2} B_{1}^{2}}+\frac{6 p^{2} Q_{2} Q_{3} z}{A_{4}^{3 / 2} B_{2}^{2}}-\frac{6 p^{2} Q_{1} Q_{4} z}{A_{1}^{3 / 2} B_{1}^{2}}-\frac{6 p^{2} Q_{2} Q_{4} z}{A_{3}^{3 / 2} B_{2}^{2}} \\
& -\frac{4 p^{2} Q_{1} Q_{3} S_{1} z}{A_{2}^{3 / 2} B_{1}^{3}}-\frac{3 p^{2} Q_{1} Q_{3} S_{1} z}{A_{2}^{5 / 2} B_{1}^{2}}+\frac{4 p^{2} Q_{2} Q_{4} S_{10} z}{A_{3}^{3 / 2} B_{2}^{3}}+\frac{3 p^{2} Q_{2} Q_{4} S_{10} z}{A_{3}^{5 / 2} B_{2}^{2}} \\
& \left.-\frac{4 p^{2} Q_{2} Q_{3} S_{2} z}{A_{4}^{3 / 2} B_{2}^{3}}-\frac{3 p^{2} Q_{2} Q_{3} S_{2} z}{A_{4}^{5 / 2} B_{2}^{2}}+\frac{4 p^{2} Q_{1} Q_{4} S_{9} z}{A_{1}^{3 / 2} B_{1}^{3}}+\frac{3 p^{2} Q_{1} Q_{4} S_{9} z}{A_{1}^{5 / 2} B_{1}^{2}}\right) \\
& -\frac{1}{K_{2}} 2\left(\frac { 1 } { 4 K \pi } b _ { z } c \left(-\frac{M_{2} p Q_{1} Q_{3}}{A_{2}^{3 / 2}}-\frac{M_{3} p Q_{2} Q_{3}}{A_{4}^{3 / 2}}+\frac{M_{4} p Q_{1} Q_{4}}{A_{1}^{3 / 2}}+\frac{M_{1} p Q_{2} Q_{4}}{A_{3}^{3 / 2}}\right.\right. \\
& +K_{2}\left(\frac{4 p Q_{1} Q_{3}}{\sqrt{A_{2}} B_{1} C_{1}}-\frac{2 H_{5} p Q_{1} Q_{3}}{\sqrt{A_{2}} B_{1} C_{1}^{2}}-\frac{2 H_{5} p Q_{1} Q_{3}}{\sqrt{A_{2}} B_{1}^{2} C_{1}}-\frac{H_{5} p Q_{1} Q_{3}}{A_{2}^{3 / 2} B_{1} C_{1}}+\frac{4 p Q_{2} Q_{3}}{\sqrt{A_{4}} B_{2} C_{1}}\right. \\
& -\frac{2 H_{6} p Q_{2} Q_{3}}{\sqrt{A_{4}} B_{2} C_{1}^{2}}-\frac{2 H_{6} p Q_{2} Q_{3}}{\sqrt{A_{4}} B_{2}^{2} C_{1}}-\frac{H_{6} p Q_{2} Q_{3}}{A_{4}^{3 / 2} B_{2} C_{1}}+\frac{2 H_{7} p Q_{1} Q_{4}}{\sqrt{A_{1}} B_{1} S_{11}^{2}}+\frac{2 H_{8} p Q_{2} Q_{4}}{\sqrt{A_{3}} B_{2} S_{11}^{2}} \\
& -\frac{4 p Q_{1} Q_{4}}{\sqrt{A_{1}} B_{1} S_{11}}+\frac{2 H_{7} p Q_{1} Q_{4}}{\sqrt{A_{1}} B_{1}^{2} S_{11}}+\frac{H_{7} p Q_{1} Q_{4}}{A_{1}^{3 / 2} B_{1} S_{11}}-\frac{4 p Q_{2} Q_{4}}{\sqrt{A_{3}} B_{2} S_{11}}+\frac{2 H_{8} p Q_{2} Q_{4}}{\sqrt{A_{3}} B_{2}^{2} S_{11}} \\
& \left.+\frac{H_{8} p Q_{2} Q_{4}}{A_{3}^{3 / 2} B_{2} S_{11}}\right)-\frac{M_{2} Q_{1} Q_{3} z}{A_{2}^{3 / 2}}-\frac{M_{6} p Q_{1} Q_{3} z}{A_{2}^{3 / 2}}+\frac{3 M_{2} p^{2} Q_{1} Q_{3} z}{A_{2}^{5 / 2}}-\frac{M_{3} Q_{2} Q_{3} z}{A_{4}^{3 / 2}} \\
& -\frac{M_{7} p Q_{2} Q_{3} z}{A_{4}^{3 / 2}}+\frac{3 M_{3} p^{2} Q_{2} Q_{3} z}{A_{4}^{5 / 2}}+\frac{M_{4} Q_{1} Q_{4} z}{A_{1}^{3 / 2}}+\frac{M_{8} p Q_{1} Q_{4} z}{A_{1}^{3 / 2}}-\frac{3 M_{4} p^{2} Q_{1} Q_{4} z}{A_{1}^{5 / 2}} \\
& \left.+\frac{M_{1} Q_{2} Q_{4} z}{A_{3}^{3 / 2}}+\frac{M_{5} p Q_{2} Q_{4} z}{A_{3}^{3 / 2}}-\frac{3 M_{1} p^{2} Q_{2} Q_{4} z}{A_{3}^{5 / 2}}\right)+\frac{1}{4 K \pi} b_{z} c\left(-\frac{G_{2} M_{10}}{C_{1}}+\frac{G_{2} M_{12}}{C_{2}}\right. \\
& +\frac{2 G_{2} M_{9} Q_{3}}{C_{1}^{2}}-\frac{2 G_{2} M_{11} Q_{4}}{C_{2}^{2}}+\frac{6 p^{2} Q_{1} Q_{3} z}{A_{2}^{3 / 2} C_{1}^{2}}+\frac{6 p^{2} Q_{2} Q_{3} z}{A_{4}^{3 / 2} C_{1}^{2}}-\frac{6 p^{2} Q_{1} Q_{4} z}{A_{1}^{3 / 2} C_{2}^{2}} \\
& -\frac{6 p^{2} Q_{2} Q_{4} z}{A_{3}^{3 / 2} C_{2}^{2}}+\frac{4 p^{2} Q_{1} Q_{4} S_{5} z}{A_{1}^{3 / 2} C_{2}^{3}}+\frac{3 p^{2} Q_{1} Q_{4} S_{5} z}{A_{1}^{5 / 2} C_{2}^{2}}-\frac{4 p^{2} Q_{1} Q_{3} S_{6} z}{A_{2}^{3 / 2} C_{1}^{3}}-\frac{3 p^{2} Q_{1} Q_{3} S_{6} z}{A_{2}^{5 / 2} C_{1}^{2}} \\
& \left.+\frac{4 p^{2} Q_{2} Q_{4} S_{7} z}{A_{3}^{3 / 2} C_{2}^{3}}+\frac{3 p^{2} Q_{2} Q_{4} S_{7} z}{A_{3}^{5 / 2} C_{2}^{2}}-\frac{4 p^{2} Q_{2} Q_{3} S_{8} z}{A_{4}^{3 / 2} C_{1}^{3}}-\frac{3 p^{2} Q_{2} Q_{3} S_{8} z}{A_{4}^{5 / 2} C_{1}^{2}}\right) \\
& +\frac{1}{4 K \pi} b_{z} c\left(G _ { 2 } \left(\frac{2 Q_{1} Q_{3}}{\sqrt{A_{2}} B_{1}^{2}}+\frac{Q_{1} Q_{3}}{A_{2}^{3 / 2} B_{1}}+\frac{2 Q_{2} Q_{3}}{\sqrt{A_{4}} B_{2}^{2}}+\frac{Q_{2} Q_{3}}{A_{4}^{3 / 2} B_{2}}-\frac{2 Q_{1} Q_{4}}{\sqrt{A_{1}} B_{1}^{2}}-\frac{Q_{1} Q_{4}}{A_{1}^{3 / 2} B_{1}}\right.\right. \\
& \left.-\frac{2 Q_{2} Q_{4}}{\sqrt{A_{3}} B_{2}^{2}}-\frac{Q_{2} Q_{4}}{A_{3}^{3 / 2} B_{2}}\right)+\frac{6 p^{2} Q_{1} Q_{3} z}{A_{2}^{3 / 2} B_{1}^{2}}+\frac{6 p^{2} Q_{2} Q_{3} z}{A_{4}^{3 / 2} B_{2}^{2}}-\frac{6 p^{2} Q_{1} Q_{4} z}{A_{1}^{3 / 2} B_{1}^{2}}-\frac{6 p^{2} Q_{2} Q_{4} z}{A_{3}^{3 / 2} B_{2}^{2}} \\
& -\frac{4 p^{2} Q_{1} Q_{3} S_{1} z}{A_{2}^{3 / 2} B_{1}^{2}}-\frac{3 p^{2} Q_{1} Q_{3} S_{1} z}{A_{2}^{5 / 2} B_{1}^{2}}+\frac{4 p^{2} Q_{2} Q_{4} S_{10} z}{A_{3}^{3 / 2} B_{2}^{3}}+\frac{3 p^{2} Q_{2} Q_{4} S_{10} z}{A_{3}^{5 / 2} B_{2}^{2}} \\
& \left.\left.-\frac{4 p^{2} Q_{2} Q_{3} S_{2} z}{A_{4}^{3 / 2} B_{2}^{3}}-\frac{3 p^{2} Q_{2} Q_{3} S_{2} z}{A_{4}^{5 / 2} B_{2}^{2}}+\frac{4 p^{2} Q_{1} Q_{4} S_{9} z}{A_{1}^{3 / 2} B_{1}^{3}}+\frac{3 p^{2} Q_{1} Q_{4} S_{9} z}{A_{1}^{5 / 2} B_{1}^{2}}\right)\right) v
\end{aligned}
$$




$$
\begin{aligned}
& \frac{\sigma_{y y}}{\mu}=\frac{1}{2 K \pi} b_{z} c\left(-\frac{G_{2} M_{10}}{C_{1}}+\frac{G_{2} M_{12}}{C_{2}}+\frac{2 G_{2} M_{9} Q_{3}}{C_{1}^{2}}-\frac{2 G_{2} M_{11} Q_{4}}{C_{2}^{2}}+\frac{6 p^{2} Q_{1} Q_{3} z}{A_{2}^{3 / 2} C_{1}^{2}}\right. \\
& +\frac{6 p^{2} Q_{2} Q_{3} z}{A_{4}^{3 / 2} C_{1}^{2}}-\frac{6 p^{2} Q_{1} Q_{4} z}{A_{1}^{3 / 2} C_{2}^{2}}-\frac{6 p^{2} Q_{2} Q_{4} z}{A_{3}^{3 / 2} C_{2}^{2}}+\frac{4 p^{2} Q_{1} Q_{4} S_{5} z}{A_{1}^{3 / 2} C_{2}^{3}}+\frac{3 p^{2} Q_{1} Q_{4} S_{5} z}{A_{1}^{5 / 2} C_{2}^{2}} \\
& -\frac{4 p^{2} Q_{1} Q_{3} S_{6} z}{A_{2}^{3 / 2} C_{1}^{3}}-\frac{3 p^{2} Q_{1} Q_{3} S_{6} z}{A_{2}^{5 / 2} C_{1}^{2}}+\frac{4 p^{2} Q_{2} Q_{4} S_{7} z}{A_{3}^{3 / 2} C_{2}^{3}}+\frac{3 p^{2} Q_{2} Q_{4} S_{7} z}{A_{3}^{3 / 2} C_{2}^{2}} \\
& \left.-\frac{4 p^{2} Q_{2} Q_{3} S_{8} z}{A_{4}^{3 / 2} C_{1}^{3}}-\frac{3 p^{2} Q_{2} Q_{3} S_{8} z}{A_{4}^{5 / 2} C_{1}^{2}}\right)-\frac{1}{K_{2}} 2\left(\frac { 1 } { 4 K \pi } b _ { z } c \left(-\frac{M_{2} p Q_{1} Q_{3}}{A_{2}^{3 / 2}}\right.\right. \\
& -\frac{M_{3} p Q_{2} Q_{3}}{A_{4}^{3 / 2}}+\frac{M_{4} p Q_{1} Q_{4}}{A_{1}^{3 / 2}}+\frac{M_{1} p Q_{2} Q_{4}}{A_{3}^{3 / 2}}+K_{2}\left(\frac{4 p Q_{1} Q_{3}}{\sqrt{A_{2}} B_{1} C_{1}}-\frac{2 H_{5} p Q_{1} Q_{3}}{\sqrt{A_{2}} B_{1} C_{1}^{2}}\right. \\
& -\frac{2 H_{5} p Q_{1} Q_{3}}{\sqrt{A_{2}} B_{1}^{2} C_{1}}-\frac{H_{5} p Q_{1} Q_{3}}{A_{2}^{3 / 2} B_{1} C_{1}}+\frac{4 p Q_{2} Q_{3}}{\sqrt{A_{4}} B_{2} C_{1}}-\frac{2 H_{6} p Q_{2} Q_{3}}{\sqrt{A_{4}} B_{2} C_{1}^{2}}-\frac{2 H_{6} p Q_{2} Q_{3}}{\sqrt{A_{4}} B_{2}^{2} C_{1}} \\
& -\frac{H_{6} p Q_{2} Q_{3}}{A_{4}^{3 / 2} B_{2} C_{1}}+\frac{2 H_{7} p Q_{1} Q_{4}}{\sqrt{A_{1}} B_{1} S_{11}^{2}}+\frac{2 H_{8} p Q_{2} Q_{4}}{\sqrt{A_{3}} B_{2} S_{11}^{2}}-\frac{4 p Q_{1} Q_{4}}{\sqrt{A_{1}} B_{1} S_{11}}+\frac{2 H_{7} p Q_{1} Q_{4}}{\sqrt{A_{1}} B_{1}^{2} S_{11}} \\
& \left.+\frac{H_{7} p Q_{1} Q_{4}}{A_{1}^{3 / 2} B_{1} S_{11}}-\frac{4 p Q_{2} Q_{4}}{\sqrt{A_{3}} B_{2} S_{11}}+\frac{2 H_{8} p Q_{2} Q_{4}}{\sqrt{A_{3}} B_{2}^{2} S_{11}}+\frac{H_{8} p Q_{2} Q_{4}}{A_{3}^{3 / 2} B_{2} S_{11}}\right)-\frac{M_{2} Q_{1} Q_{3} z}{A_{2}^{3 / 2}} \\
& -\frac{M_{6} p Q_{1} Q_{3} z}{A_{2}^{3 / 2}}+\frac{3 M_{2} p^{2} Q_{1} Q_{3} z}{A_{2}^{5 / 2}}-\frac{M_{3} Q_{2} Q_{3} z}{A_{4}^{3 / 2}}-\frac{M_{7} p Q_{2} Q_{3} z}{A_{4}^{3 / 2}}+\frac{3 M_{3} p^{2} Q_{2} Q_{3} z}{A_{4}^{5 / 2}} \\
& +\frac{M 4 Q_{1} Q_{4} z}{A_{1}^{3 / 2}}+\frac{M_{8} p Q_{1} Q_{4} z}{A_{1}^{3 / 2}}-\frac{3 M_{4} p^{2} Q_{1} Q_{4} z}{A_{1}^{5 / 2}}+\frac{M_{1} Q_{2} Q_{4} z}{A_{3}^{3 / 2}}+\frac{M_{5} p Q_{2} Q_{4} z}{A_{3}^{3 / 2}} \\
& \left.-\frac{3 M_{1} p^{2} Q_{2} Q_{4} z}{A_{3}^{5 / 2}}\right)+\frac{1}{4 K \pi} b_{z} c\left(-\frac{G_{2} M_{10}}{C_{1}}+\frac{G_{2} M_{12}}{C_{2}}+\frac{2 G_{2} M_{9} Q_{3}}{C_{1}^{2}}\right. \\
& -\frac{2 G_{2} M_{11} Q_{4}}{C_{2}^{2}}+\frac{6 p^{2} Q_{1} Q_{3} z}{A_{2}^{3 / 2} C_{1}^{2}}+\frac{6 p^{2} Q_{2} Q_{3} z}{A_{4}^{3 / 2} C_{1}^{2}}-\frac{6 p^{2} Q_{1} Q_{4} z}{A_{1}^{3 / 2} C_{2}^{2}}-\frac{6 p^{2} Q_{2} Q_{4} z}{A_{3}^{3 / 2} C_{2}^{2}} \\
& +\frac{4 p^{2} Q_{1} Q_{4} S_{5} z}{A_{1}^{3 / 2} C_{2}^{3}}+\frac{3 p^{2} Q_{1} Q_{4} S_{5} z}{A_{1}^{5 / 2} C_{2}^{2}}-\frac{4 p^{2} Q_{1} Q_{3} S_{6} z}{A_{2}^{3 / 2} C_{1}^{3}}-\frac{3 p^{2} Q_{1} Q_{3} S_{6} z}{A_{2}^{5 / 2} C_{1}^{2}} \\
& \left.+\frac{4 p^{2} Q_{2} Q_{4} S_{7} z}{A_{3}^{3 / 2} C_{2}^{2}}+\frac{3 p^{2} Q_{2} Q_{4} S_{7} z}{A_{3}^{5 / 2} C_{2}^{2}}-\frac{4 p^{2} Q_{2} Q_{3} S_{8} z}{A_{4}^{3 / 2} C_{1}^{3}}-\frac{3 p^{2} Q_{2} Q_{3} S_{8} z}{A_{4}^{5 / 2} C_{1}^{2}}\right) \\
& +\frac{1}{4 K \pi} b_{z} c\left(G _ { 2 } \left(\frac{2 Q_{1} Q_{3}}{\sqrt{A_{2}} B_{1}^{2}}+\frac{Q_{1} Q_{3}}{A_{2}^{3 / 2} B_{1}}+\frac{2 Q_{2} Q_{3}}{\sqrt{A_{4}} B_{2}^{2}}+\frac{Q_{2} Q_{3}}{A_{4}^{3 / 2} B_{2}}-\frac{2 Q_{1} Q_{4}}{\sqrt{A_{1}} B_{1}^{2}}-\frac{Q_{1} Q_{4}}{A_{1}^{3 / 2} B_{1}}\right.\right. \\
& \left.-\frac{2 Q_{2} Q_{4}}{\sqrt{A_{3}} B_{2}^{2}}-\frac{Q_{2} Q_{4}}{A_{3}^{3 / 2} B_{2}}\right)+\frac{6 p^{2} Q_{1} Q_{3} z}{A_{2}^{3 / 2} B_{1}^{2}}+\frac{6 p^{2} Q_{2} Q_{3} z}{A_{4}^{3 / 2} B_{2}^{2}}-\frac{6 p^{2} Q_{1} Q_{4} z}{A_{1}^{3 / 2} B_{1}^{2}}-\frac{6 p^{2} Q_{2} Q_{4} z}{A_{3}^{3 / 2} B_{2}^{2}} \\
& -\frac{4 p^{2} Q_{1} Q_{3} S_{1} z}{A_{2}^{3 / 2} B_{1}^{3}}-\frac{3 p^{2} Q_{1} Q_{3} S_{1} z}{A_{2}^{5 / 2} B_{1}^{2}}+\frac{4 p^{2} Q_{2} Q_{4} S_{10} z}{A_{3}^{3 / 2} B_{2}^{3}}+\frac{3 p^{2} Q_{2} Q_{4} S_{10} z}{A_{3}^{5 / 2} B_{2}^{2}} \\
& \left.\left.-\frac{4 p^{2} Q_{2} Q_{3} S_{2} z}{A_{4}^{3 / 2} B_{2}^{3}}-\frac{3 p^{2} Q_{2} Q_{3} S_{2} z}{A_{4}^{5 / 2} B_{2}^{2}}+\frac{4 p^{2} Q_{1} Q_{4} S_{9} z}{A_{1}^{3 / 2} B_{1}^{3}}+\frac{3 p^{2} Q_{1} Q_{4} S_{9} z}{A_{1}^{5 / 2} B_{1}^{2}}\right)\right) v \\
& \frac{\sigma_{z z}}{\mu}=\frac{1}{2 K \pi} b_{z} c\left(-\frac{M_{2} p Q_{1} Q_{3}}{A_{2}^{3 / 2}}-\frac{M_{3} p Q_{2} Q_{3}}{A_{4}^{3 / 2}}+\frac{M_{4} p Q_{1} Q_{4}}{A_{1}^{3 / 2}}+\frac{M_{1} p Q_{2} Q_{4}}{A_{3}^{3 / 2}}\right. \\
& +K_{2}\left(\frac{4 p Q_{1} Q_{3}}{\sqrt{A_{2}} B_{1} C_{1}}-\frac{2 H_{5} p Q_{1} Q_{3}}{\sqrt{A_{2}} B_{1} C_{1}^{2}}-\frac{2 H_{5} p Q_{1} Q_{3}}{\sqrt{A_{2}} B_{1}^{2} C_{1}}-\frac{H_{5} p Q_{1} Q_{3}}{A_{2}^{3 / 2} B_{1} C_{1}}+\frac{4 p Q_{2} Q_{3}}{\sqrt{A_{4}} B_{2} C_{1}}\right.
\end{aligned}
$$




$$
\begin{aligned}
& -\frac{2 H_{6} p Q_{2} Q_{3}}{\sqrt{A_{4}} B_{2} C_{1}^{2}}-\frac{2 H_{6} p Q_{2} Q_{3}}{\sqrt{A_{4}} B_{2}^{2} C_{1}}-\frac{H_{6} p Q_{2} Q_{3}}{A_{4}^{3 / 2} B_{2} C_{1}}+\frac{2 H_{7} p Q_{1} Q_{4}}{\sqrt{A_{1}} B_{1} S_{11}^{2}}+\frac{2 H_{8} p Q_{2} Q_{4}}{\sqrt{A_{3}} B_{2} S_{11}^{2}} \\
& -\frac{4 p Q_{1} Q_{4}}{\sqrt{A_{1}} B_{1} S_{11}}+\frac{2 H_{7} p Q_{1} Q_{4}}{\sqrt{A_{1}} B_{1}^{2} S_{11}}+\frac{H_{7} p Q_{1} Q_{4}}{A_{1}^{3 / 2} B_{1} S_{11}}-\frac{4 p Q_{2} Q_{4}}{\sqrt{A_{3}} B_{2} S_{11}}+\frac{2 H_{8} p Q_{2} Q_{4}}{\sqrt{A_{3}} B_{2}^{2} S_{11}} \\
& \left.+\frac{H_{8} p Q_{2} Q_{4}}{A_{3}^{3 / 2} B_{2} S_{11}}\right)-\frac{M_{2} Q_{1} Q_{3} z}{A_{2}^{3 / 2}}-\frac{M_{6} p Q_{1} Q_{3} z}{A_{2}^{3 / 2}}+\frac{3 M_{2} p^{2} Q_{1} Q_{3} z}{A_{2}^{5 / 2}}-\frac{M_{3} Q_{2} Q_{3} z}{A_{4}^{3 / 2}} \\
& -\frac{M_{7} p Q_{2} Q_{3} z}{A_{4}^{3 / 2}}+\frac{3 M_{3} p^{2} Q_{2} Q_{3} z}{A_{4}^{5 / 2}}+\frac{M_{4} Q_{1} Q_{4} z}{A_{1}^{3 / 2}}+\frac{M_{8} p Q_{1} Q_{4} z}{A_{1}^{3 / 2}}-\frac{3 M_{4} p^{2} Q_{1} Q_{4} z}{A_{1}^{5 / 2}} \\
& \left.+\frac{M_{1} Q_{2} Q_{4} z}{A_{3}^{3 / 2}}+\frac{M_{5} p Q_{2} Q_{4} z}{A_{3}^{3 / 2}}-\frac{3 M_{1} p^{2} Q_{2} Q_{4} z}{A_{3}^{5 / 2}}\right)-\frac{1}{K_{2}} 2\left(\frac { 1 } { 4 K \pi } b _ { z } c \left(-\frac{M_{2} p Q_{1} Q_{3}}{A_{2}^{3 / 2}}\right.\right. \\
& -\frac{M_{3} p Q_{2} Q_{3}}{A_{4}^{3 / 2}}+\frac{M_{4} p Q_{1} Q_{4}}{A_{1}^{3 / 2}}+\frac{M_{1} p Q_{2} Q_{4}}{A_{3}^{3 / 2}}+K_{2}\left(\frac{4 p Q_{1} Q_{3}}{\sqrt{A_{2}} B_{1} C_{1}}-\frac{2 H_{5} p Q_{1} Q_{3}}{\sqrt{A_{2}} B_{1} C_{1}^{2}}\right. \\
& -\frac{2 H_{5} p Q_{1} Q_{3}}{\sqrt{A_{2}} B_{1}^{2} C_{1}}-\frac{H_{5} p Q_{1} Q_{3}}{A_{2}^{3 / 2} B_{1} C_{1}}+\frac{4 p Q_{2} Q_{3}}{\sqrt{A_{4}} B_{2} C_{1}}-\frac{2 H_{6} p Q_{2} Q_{3}}{\sqrt{A_{4}} B_{2} C_{1}^{2}}-\frac{2 H_{6} p Q_{2} Q_{3}}{\sqrt{A_{4}} B_{2}^{2} C_{1}} \\
& -\frac{H_{6} p Q_{2} Q_{3}}{A_{4}^{3 / 2} B_{2} C_{1}}+\frac{2 H_{7} p Q_{1} Q_{4}}{\sqrt{A_{1}} B_{1} S_{11}^{2}}+\frac{2 H_{8} p Q_{2} Q_{4}}{\sqrt{A_{3}} B_{2} S_{11}^{2}}-\frac{4 p Q_{1} Q_{4}}{\sqrt{A_{1}} B_{1} S_{11}}+\frac{2 H_{7} p Q_{1} Q_{4}}{\sqrt{A_{1}} B_{1}^{2} S_{11}} \\
& \left.+\frac{H_{7} p Q_{1} Q_{4}}{A_{1}^{3 / 2} B_{1} S_{11}}-\frac{4 p Q_{2} Q_{4}}{\sqrt{A_{3}} B_{2} S_{11}}+\frac{2 H_{8} p Q_{2} Q_{4}}{\sqrt{A_{3}} B_{2}^{2} S_{11}}+\frac{H_{8} p Q_{2} Q_{4}}{A_{3}^{3 / 2} B_{2} S_{11}}\right)-\frac{M_{2} Q_{1} Q_{3} z}{A_{2}^{3 / 2}} \\
& -\frac{M_{6} p Q_{1} Q_{3} z}{A_{2}^{3 / 2}}+\frac{3 M_{2} p^{2} Q_{1} Q_{3} z}{A_{2}^{5 / 2}}-\frac{M_{3} Q_{2} Q_{3} z}{A_{4}^{3 / 2}}-\frac{M_{7} p Q_{2} Q_{3} z}{A_{4}^{3 / 2}}+\frac{3 M_{3} p^{2} Q_{2} Q_{3} z}{A_{4}^{5 / 2}} \\
& +\frac{M_{4} Q_{1} Q_{4} z}{A_{1}^{3 / 2}}+\frac{M_{8} p Q_{1} Q_{4} z}{A_{1}^{3 / 2}}-\frac{3 M_{4} p^{2} Q_{1} Q_{4} z}{A_{1}^{5 / 2}}+\frac{M_{1} Q_{2} Q_{4} z}{A_{3}^{3 / 2}}+\frac{M_{5} p Q_{2} Q_{4} z}{A_{3}^{3 / 2}} \\
& \left.-\frac{3 M_{1} p^{2} Q_{2} Q_{4} z}{A_{3}^{5 / 2}}\right)+\frac{1}{4 K \pi} b_{z} c\left(-\frac{G_{2} M_{10}}{C_{1}}+\frac{G_{2} M_{12}}{C_{2}}+\frac{2 G_{2} M_{9} Q_{3}}{C_{1}^{2}}\right. \\
& -\frac{2 G_{2} M_{11} Q_{4}}{C_{2}^{2}}+\frac{6 p^{2} Q_{1} Q_{3} z}{A_{2}^{3 / 2} C_{1}^{2}}+\frac{6 p^{2} Q_{2} Q_{3} z}{A_{4}^{3 / 2} C_{1}^{2}}-\frac{6 p^{2} Q_{1} Q_{4} z}{A_{1}^{3 / 2} C_{2}^{2}}-\frac{6 p^{2} Q_{2} Q_{4} z}{A_{3}^{3 / 2} C_{2}^{2}} \\
& +\frac{4 p^{2} Q_{1} Q_{4} S_{5} z}{A_{1}^{3 / 2} C_{2}^{3}}+\frac{3 p^{2} Q_{1} Q_{4} S_{5} z}{A_{1}^{5 / 2} C_{2}^{2}}-\frac{4 p^{2} Q_{1} Q_{3} S_{6} z}{A_{2}^{3 / 2} C_{1}^{3}}-\frac{3 p^{2} Q_{1} Q_{3} S_{6} z}{A_{2}^{5 / 2} C_{1}^{2}} \\
& \left.+\frac{4 p^{2} Q_{2} Q_{4} S_{7} z}{A_{3}^{3 / 2} C_{2}^{3}}+\frac{3 p^{2} Q_{2} Q_{4} S_{7} z}{A_{3}^{5 / 2} C_{2}^{2}}-\frac{4 p^{2} Q_{2} Q_{3} S_{8} z}{A_{4}^{3 / 2} C_{1}^{3}}-\frac{3 p^{2} Q_{2} Q_{3} S_{8} z}{A_{4}^{5 / 2} C_{1}^{2}}\right) \\
& +\frac{1}{4 K \pi} b_{z} c\left(G _ { 2 } \left(\frac{2 Q_{1} Q_{3}}{\sqrt{A_{2}} B_{1}^{2}}+\frac{Q_{1} Q_{3}}{A_{2}^{3 / 2} B_{1}}+\frac{2 Q_{2} Q_{3}}{\sqrt{A_{4}} B_{2}^{2}}+\frac{Q_{2} Q_{3}}{A_{4}^{3 / 2} B_{2}}-\frac{2 Q_{1} Q_{4}}{\sqrt{A_{1}} B_{1}^{2}}-\frac{Q_{1} Q_{4}}{A_{1}^{3 / 2} B_{1}}\right.\right. \\
& \left.-\frac{2 Q_{2} Q_{4}}{\sqrt{A_{3}} B_{2}^{2}}-\frac{Q_{2} Q_{4}}{A_{3}^{3 / 2} B_{2}}\right)+\frac{6 p^{2} Q_{1} Q_{3} z}{A_{2}^{3 / 2} B_{1}^{2}}+\frac{6 p^{2} Q_{2} Q_{3} z}{A_{4}^{3 / 2} B_{2}^{2}}-\frac{6 p^{2} Q_{1} Q_{4} z}{A_{1}^{3 / 2} B_{1}^{2}}-\frac{6 p^{2} Q_{2} Q_{4} z}{A_{3}^{3 / 2} B_{2}^{2}} \\
& -\frac{4 p^{2} Q_{1} Q_{3} S_{1} z}{A_{2}^{3 / 2} B_{1}^{3}}-\frac{3 p^{2} Q_{1} Q_{3} S_{1} z}{A_{2}^{5 / 2} B_{1}^{2}}+\frac{4 p^{2} Q_{2} Q_{4} S_{10} z}{A_{3}^{3 / 2} B_{2}^{3}}+\frac{3 p^{2} Q_{2} Q_{4} S_{10} z}{A_{3}^{5 / 2} B_{2}^{2}} \\
& \left.\left.-\frac{4 p^{2} Q_{2} Q_{3} S_{2} z}{A_{4}^{3 / 2} B_{2}^{3}}-\frac{3 p^{2} Q_{2} Q_{3} S_{2} z}{A_{4}^{5 / 2} B_{2}^{2}}+\frac{4 p^{2} Q_{1} Q_{4} S_{9} z}{A_{1}^{3 / 2} B_{1}^{3}}+\frac{3 p^{2} Q_{1} Q_{4} S_{9} z}{A_{1}^{5 / 2} B_{1}^{2}}\right)\right) v
\end{aligned}
$$




$$
\begin{aligned}
& \frac{\sigma_{x y}}{\mu}=\frac{1}{4 K \pi} b_{z} c\left(\frac{G_{2}}{C_{2}}\left(-\frac{1}{\sqrt{A_{1}}}+\frac{1}{\sqrt{A_{3}}}+\frac{Q_{1}^{2}}{A_{1}^{3 / 2}}-\frac{Q_{2}^{2}}{A_{3}^{3 / 2}}\right)-\frac{G_{2}}{C_{1}}\left(-\frac{1}{\sqrt{A_{2}}}+\frac{1}{\sqrt{A_{4}}}\right.\right. \\
& \left.+\frac{Q_{1}^{2}}{A_{2}^{3 / 2}}-\frac{Q_{2}^{2}}{A_{4}^{3 / 2}}\right)-\frac{4 p^{2} Q_{1}^{2} z}{A_{2}^{3 / 2} C_{1}^{2}}+\frac{4 p^{2} Q_{1}^{2} z}{A_{1}^{3 / 2} C_{2}^{2}}+\frac{4 p^{2} Q_{2}^{2} z}{A_{4}^{3 / 2} C_{1}^{2}}-\frac{4 p^{2} Q_{2}^{2} z}{A_{3}^{3 / 2} C_{2}^{2}}+\frac{p^{2} S_{5} z}{A_{1}^{3 / 2} C_{2}^{2}} \\
& -\frac{3 p^{2} Q_{1}^{2} S_{5} z}{A_{1}^{5 / 2} C_{2}^{2}}-\frac{p^{2} S_{6} z}{A_{2}^{3 / 2} C_{1}^{2}}+\frac{3 p^{2} Q_{1}^{2} S_{6} z}{A_{2}^{5 / 2} C_{1}^{2}}-\frac{p^{2} S_{7} z}{A_{3}^{3 / 2} C_{2}^{2}}+\frac{3 p^{2} Q_{2}^{2} S_{7} z}{A_{3}^{5 / 2} C_{2}^{2}}+\frac{p^{2} S_{8} z}{A_{4}^{3 / 2} C_{1}^{2}} \\
& \left.-\frac{3 p^{2} Q_{2}^{2} S_{8} z}{A_{4}^{5 / 2} C_{1}^{2}}\right)+\frac{1}{4 K \pi} b_{z} c\left(G _ { 2 } \left(-\frac{1}{\sqrt{A_{1}} B_{1}}+\frac{1}{\sqrt{A_{2}} B_{1}}+\frac{1}{\sqrt{A_{3}} B_{2}}-\frac{1}{\sqrt{A_{4}} B_{2}}\right.\right. \\
& \left.-\frac{Q_{3}^{2}}{A_{2}^{3 / 2} B_{1}}+\frac{Q_{3}^{2}}{A_{4}^{3 / 2} B_{2}}+\frac{Q_{4}^{2}}{A_{1}^{3 / 2} B_{1}}-\frac{Q_{4}^{2}}{A_{3}^{3 / 2} B_{2}}\right)-\frac{4 p^{2} Q_{3}^{2} z}{A_{2}^{3 / 2} B_{1}^{2}}+\frac{4 p^{2} Q_{3}^{2} z}{A_{4}^{3 / 2} B_{2}^{2}}+\frac{4 p^{2} Q_{4}^{2} z}{A_{1}^{3 / 2} B_{1}^{2}} \\
& -\frac{4 p^{2} Q_{4}^{2} z}{A_{3}^{3 / 2} B_{2}^{2}}-\frac{p^{2} S_{1} z}{A_{2}^{3 / 2} B_{1}^{2}}+\frac{3 p^{2} Q_{3}^{2} S_{1} z}{A_{2}^{5 / 2} B_{1}^{2}}-\frac{p^{2} S_{10} z}{A_{3}^{3 / 2} B_{2}^{2}}+\frac{3 p^{2} Q_{4}^{2} S_{10} z}{A_{3}^{5 / 2} B_{2}^{2}}+\frac{p^{2} S_{2} z}{A_{4}^{3 / 2} B_{2}^{2}} \\
& \left.-\frac{3 p^{2} Q_{3}^{2} S_{2} z}{A_{4}^{5 / 2} B_{2}^{2}}+\frac{p^{2} S_{9} z}{A_{1}^{3 / 2} B_{1}^{2}}-\frac{3 p^{2} Q_{4}^{2} S_{9} z}{A_{1}^{5 / 2} B_{1}^{2}}\right) \\
& \frac{\sigma_{x z}}{\mu}=\frac{1}{4 K \pi} b_{z} c\left((1+2 K)\left(\frac{Q_{3}}{\sqrt{A_{2}} B_{1}}-\frac{Q_{3}}{\sqrt{A_{4}} B_{2}}-\frac{Q_{4}}{\sqrt{A_{1}} B_{1}}+\frac{Q_{4}}{\sqrt{A_{3}} B_{2}}\right)\right. \\
& +G_{2}\left(-\frac{2 p Q_{3}}{\sqrt{A_{2}} B_{1}^{2}}-\frac{p Q_{3}}{A_{2}^{3 / 2} B_{1}}+\frac{2 p Q_{3}}{\sqrt{A_{4}} B_{2}^{2}}+\frac{p Q_{3}}{A_{4}^{3 / 2} B_{2}}+\frac{2 p Q_{4}}{\sqrt{A_{1}} B_{1}^{2}}+\frac{p Q_{4}}{A_{1}^{3 / 2} B_{1}}\right. \\
& \left.-\frac{2 p Q_{4}}{\sqrt{A_{3}} B_{2}^{2}}-\frac{p Q_{4}}{A_{3}^{3 / 2} B_{2}}\right)-\frac{p^{2} Q_{3} S_{1}}{A_{2}^{3 / 2} B_{1}^{2}}-\frac{p^{2} Q_{4} S_{10}}{A_{3}^{3 / 2} B_{2}^{2}}+\frac{p^{2} Q_{3} S_{2}}{A_{4}^{3 / 2} B_{2}^{2}}+\frac{p^{2} Q_{4} S_{9}}{A_{1}^{3 / 2} B_{1}^{2}} \\
& -\frac{6 p^{3} Q_{3} z}{A_{2}^{3 / 2} B_{1}^{2}}+\frac{6 p^{3} Q_{3} z}{A_{4}^{3 / 2} B_{2}^{2}}+\frac{6 p^{3} Q_{4} z}{A_{1}^{3 / 2} B_{1}^{2}}-\frac{6 p^{3} Q_{4} z}{A_{3}^{3 / 2} B_{2}^{2}}-\frac{2 p Q_{3} S_{1} z}{A_{2}^{3 / 2} B_{1}^{2}}+\frac{4 p^{3} Q_{3} S_{1} z}{A_{2}^{3 / 2} B_{1}^{3}} \\
& +\frac{3 p^{3} Q_{3} S_{1} z}{A_{2}^{5 / 2} B_{1}^{2}}-\frac{2 p Q_{4} S_{10} z}{A_{3}^{3 / 2} B_{2}^{2}}+\frac{4 p^{3} Q_{4} S_{10} z}{A_{3}^{3 / 2} B_{2}^{3}}+\frac{3 p^{3} Q_{4} S_{10} z}{A_{3}^{5 / 2} B_{2}^{2}}+\frac{2 p Q_{3} S_{2} z}{A_{4}^{3 / 2} B_{2}^{2}} \\
& \left.-\frac{4 p^{3} Q_{3} S_{2} z}{A_{4}^{3 / 2} B_{2}^{3}}-\frac{3 p^{3} Q_{3} S_{2} z}{A_{4}^{5 / 2} B_{2}^{2}}+\frac{2 p Q_{4} S_{9} z}{A_{1}^{3 / 2} B_{1}^{2}}-\frac{4 p^{3} Q_{4} S_{9} z}{A_{1}^{3 / 2} B_{1}^{3}}-\frac{3 p^{3} Q_{4} S_{9} z}{A_{1}^{5 / 2} B_{1}^{2}}\right) \\
& +\frac{1}{4 K \pi} b_{z} c\left(K _ { 2 } \left(-\frac{H_{5} Q_{3}}{\sqrt{A_{2}} B_{1} C_{1}}+\frac{H_{6} Q_{3}}{\sqrt{A_{4}} B_{2} C_{1}}-\frac{2 Q_{1}^{2} Q_{3}}{\sqrt{A_{2}} B_{1} C_{1}}+\frac{2 H_{5} Q_{1}^{2} Q_{3}}{\sqrt{A_{2}} B_{1}^{2} C_{1}}\right.\right. \\
& +\frac{H_{5} Q_{1}^{2} Q_{3}}{A_{2}^{3 / 2} B_{1} C_{1}}+\frac{2 Q_{2}^{2} Q_{3}}{\sqrt{A_{4}} B_{2} C_{1}}-\frac{2 H_{6} Q_{2}^{2} Q_{3}}{\sqrt{A_{4}} B_{2}^{2} C_{1}}-\frac{H_{6} Q_{2}^{2} Q_{3}}{A_{4}^{3 / 2} B_{2} C_{1}}+\frac{H_{7} Q_{4}}{\sqrt{A_{1}} B_{1} S_{11}} \\
& -\frac{H_{8} Q_{4}}{\sqrt{A_{3}} B_{2} S_{11}}+\frac{2 Q_{1}^{2} Q_{4}}{\sqrt{A_{1}} B_{1} S_{11}}-\frac{2 H_{7} Q_{1}^{2} Q_{4}}{\sqrt{A_{1}} B_{1}^{2} S_{11}}-\frac{H_{7} Q_{1}^{2} Q_{4}}{A_{1}^{3 / 2} B_{1} S_{11}}-\frac{2 Q_{2}^{2} Q_{4}}{\sqrt{A_{3}} B_{2} S_{11}} \\
& \left.+\frac{2 H_{8} Q_{2}^{2} Q_{4}}{\sqrt{A_{3}} B_{2}^{2} S_{11}}+\frac{H_{8} Q_{2}^{2} Q_{4}}{A_{3}^{3 / 2} B_{2} S_{11}}\right)+\frac{M_{2} p Q_{3} z}{A_{2}^{3 / 2}}-\frac{M_{3} p Q_{3} z}{A_{4}^{3 / 2}}-\frac{3 M_{2} p Q_{1}^{2} Q_{3} z}{A_{2}^{5 / 2}} \\
& +\frac{3 M_{3} p Q_{2}^{2} Q_{3} z}{A_{4}^{5 / 2}}+\frac{M_{1} p Q_{4} z}{A_{3}^{3 / 2}}-\frac{M_{4} p Q_{4} z}{A_{1}^{3 / 2}}+\frac{3 M_{4} p Q_{1}^{2} Q_{4} z}{A_{1}^{5 / 2}}-\frac{3 M_{1} p Q_{2}^{2} Q_{4} z}{A_{3}^{5 / 2}} \\
& -\frac{p Q_{1} Q_{3}}{A_{2}^{3 / 2}}\left(-\frac{6 Q_{1}}{B_{1}^{2}}-\frac{4 Q_{1}}{C_{1}^{2}}+\frac{4 Q_{1} S_{1}}{B_{1}^{3}}\right) z+\frac{p Q_{2} Q_{4}}{A_{3}^{3 / 2}}\left(\frac{6 Q_{2}}{B_{2}^{2}}-\frac{4 Q_{2} S_{10}}{B_{2}^{3}}+\frac{4 Q_{2}}{S_{11}^{2}}\right) z
\end{aligned}
$$




$$
\begin{aligned}
& \left.-\frac{p Q_{2} Q_{3}}{A_{4}^{3 / 2}}\left(\frac{6 Q_{2}}{B_{2}^{2}}+\frac{4 Q_{2}}{C_{1}^{2}}-\frac{4 Q_{2} S_{2}}{B_{2}^{3}}\right) z+\frac{p Q_{1} Q_{4}}{A_{1}^{3 / 2}}\left(-\frac{6 Q_{1}}{B_{1}^{2}}-\frac{4 Q_{1}}{S_{11}^{2}}+\frac{4 Q_{1} S_{9}}{B_{1}^{3}}\right) z\right) \\
& \frac{\sigma_{y z}}{\mu}=\frac{1}{4 K \pi} b_{z} c\left(\frac{(1+2 K) M_{11}}{C_{2}}-\frac{(1+2 K) M_{9}}{C_{1}}-\frac{2 G_{2} M_{11} p}{C_{2}^{2}}+\frac{2 G_{2} M_{9} p}{C_{1}^{2}}\right. \\
& +\frac{G_{2}}{C_{2}}\left(-\frac{p Q_{1}}{A_{1}^{3 / 2}}-\frac{p Q_{2}}{A_{3}^{3 / 2}}\right)-\frac{G_{2}}{C_{1}}\left(-\frac{p Q_{1}}{A_{2}^{3 / 2}}-\frac{p Q_{2}}{A_{4}^{3 / 2}}\right)-\frac{p^{2} Q_{1} S_{5}}{A_{1}^{3 / 2} C_{2}^{2}}+\frac{p^{2} Q_{1} S_{6}}{A_{2}^{3 / 2} C_{1}^{2}} \\
& -\frac{p^{2} Q_{2} S_{7}}{A_{3}^{3 / 2} C_{2}^{2}}+\frac{p^{2} Q_{2} S_{8}}{A_{4}^{3 / 2} C_{1}^{2}}+\frac{6 p^{3} Q_{1} z}{A_{2}^{3 / 2} C_{1}^{2}}-\frac{6 p^{3} Q_{1} z}{A_{1}^{3 / 2} C_{2}^{2}}+\frac{6 p^{3} Q_{2} z}{A_{4}^{3 / 2} C_{1}^{2}}-\frac{6 p^{3} Q_{2} z}{A_{3}^{3 / 2} C_{2}^{2}} \\
& -\frac{2 p Q_{1} S_{5} z}{A_{1}^{3 / 2} C_{2}^{2}}+\frac{4 p^{3} Q_{1} S_{5} z}{A_{1}^{3 / 2} C_{2}^{3}}+\frac{3 p^{3} Q_{1} S_{5} z}{A_{1}^{5 / 2} C_{2}^{2}}+\frac{2 p Q_{1} S_{6} z}{A_{2}^{3 / 2} C_{1}^{2}}-\frac{4 p^{3} Q_{1} S_{6} z}{A_{2}^{3 / 2} C_{1}^{3}} \\
& -\frac{3 p^{3} Q_{1} S_{6} z}{A_{2}^{5 / 2} C_{1}^{2}}-\frac{2 p Q_{2} S_{7} z}{A_{3}^{3 / 2} C_{2}^{2}}+\frac{4 p^{3} Q_{2} S_{7} z}{A_{3}^{3 / 2} C_{2}^{3}}+\frac{3 p^{3} Q_{2} S_{7} z}{A_{3}^{5 / 2} C_{2}^{2}}+\frac{2 p Q_{2} S_{8} z}{A_{4}^{3 / 2} C_{1}^{2}} \\
& \left.-\frac{4 p^{3} Q_{2} S_{8} z}{A_{4}^{3 / 2} C_{1}^{3}}-\frac{3 p^{3} Q_{2} S_{8} z}{A_{4}^{5 / 2} C_{1}^{2}}\right)+\frac{1}{4 K \pi} b_{z} c\left(K _ { 2 } \left(\frac{H_{5} Q_{1}}{\sqrt{A_{2} B_{1} C_{1}}}+\frac{H_{6} Q_{2}}{\sqrt{A_{4}} B_{2} C_{1}}\right.\right. \\
& +\frac{2 Q_{1} Q_{3}^{2}}{\sqrt{A_{2}} B_{1} C_{1}}-\frac{2 H_{5} Q_{1} Q_{3}^{2}}{\sqrt{A_{2}} B_{1} C_{1}^{2}}-\frac{H_{5} Q_{1} Q_{3}^{2}}{A_{2}^{3 / 2} B_{1} C_{1}}+\frac{2 Q_{2} Q_{3}^{2}}{\sqrt{A_{4}} B_{2} C_{1}}-\frac{2 H_{6} Q_{2} Q_{3}^{2}}{\sqrt{A_{4}} B_{2} C_{1}^{2}} \\
& -\frac{H_{6} Q_{2} Q_{3}^{2}}{A_{4}^{3 / 2} B_{2} C_{1}}+\frac{2 H_{7} Q_{1} Q_{4}^{2}}{\sqrt{A_{1}} B_{1} S_{11}^{2}}+\frac{2 H_{8} Q_{2} Q_{4}^{2}}{\sqrt{A_{3}} B_{2} S_{11}^{2}}-\frac{H_{7} Q_{1}}{\sqrt{A_{1}} B_{1} S_{11}}-\frac{H_{8} Q_{2}}{\sqrt{A_{3}} B_{2} S_{11}} \\
& \left.-\frac{2 Q_{1} Q_{4}^{2}}{\sqrt{A_{1}} B_{1} S_{11}}+\frac{H_{7} Q_{1} Q_{4}^{2}}{A_{1}^{3 / 2} B_{1} S_{11}}-\frac{2 Q_{2} Q_{4}^{2}}{\sqrt{A_{3}} B_{2} S_{11}}+\frac{H_{8} Q_{2} Q_{4}^{2}}{A_{2}^{3 / 2} B_{2} S_{11}}\right)-\frac{M_{2} p Q_{1} z}{A_{2}^{3 / 2}} \\
& +\frac{M_{4} p Q_{1} z}{A_{1}^{3 / 2}}+\frac{M_{1} p Q_{2} z}{A_{3}^{3 / 2}}-\frac{M_{3} p Q_{2} z}{A_{4}^{3 / 2}}+\frac{3 M_{2} p Q_{1} Q_{3}^{2} z}{A_{2}^{5 / 2}}+\frac{3 M_{3} p Q_{2} Q_{3}^{2} z}{A_{4}^{5 / 2}} \\
& -\frac{3 M_{4} p Q_{1} Q_{4}^{2} z}{A_{1}^{5 / 2}}-\frac{3 M_{1} p Q_{2} Q_{4}^{2} z}{A_{3}^{5 / 2}}+\frac{p Q_{1} Q_{4}}{A_{1}^{3 / 2}}\left(\frac{4 Q_{4}}{B_{1}^{2}}+\frac{6 Q_{4}}{S_{11}^{2}}\right. \\
& \left.-\frac{4 Q_{4}\left(2 Q_{1}^{2}+3 S_{11}\right)}{S_{11}^{3}}\right) z+\frac{p Q_{2} Q_{4}}{A_{3}^{3 / 2}}\left(\frac{4 Q_{4}}{B_{2}^{2}}+\frac{6 Q_{4}}{S_{11}^{2}}-\frac{4 Q_{4}\left(2 Q_{2}^{2}+3 S_{11}\right)}{S_{11}^{3}}\right) z \\
& \left.-\frac{p Q_{1} Q_{3}}{A_{2}^{3 / 2}}\left(\frac{4 Q_{3}}{B_{1}^{2}}+\frac{6 Q_{3}}{C_{1}^{2}}-\frac{4 Q_{3} S_{6}}{C_{1}^{3}}\right) z-\frac{p Q_{2} Q_{3}}{A_{4}^{3 / 2}}\left(\frac{4 Q_{3}}{B_{2}^{2}}+\frac{6 Q_{3}}{C_{1}^{2}}-\frac{4 Q_{3} S_{8}}{C_{1}^{3}}\right) z\right) \\
& A_{1}=(a-x)^{2}+(b-y)^{2}+(c+z)^{2} ; A_{2}=(a-x)^{2}+(b+y)^{2}+(c+z)^{2} ; \\
& A_{3}=(a+x)^{2}+(b-y)^{2}+(c+z)^{2} ; \quad A_{4}=(a+x)^{2}+(b+y)^{2}+(c+z)^{2} ; \\
& B_{1}=(a-x)^{2}+(c+z)^{2} ; \quad B_{2}=(a+x)^{2}+(c+z)^{2} ; C_{1}=(b+y)^{2}+(c+z)^{2} ; \\
& C_{2}=(b-y)^{2}+(c+z)^{2} ; p=c+z ; \quad Q_{1}=a-x ; \quad Q_{2}=a+x ; \\
& Q_{3}=b+y ; \quad Q_{4}=-b+y ; K=-1+v ; K_{2}=-1+2 v ; K_{3}=1+v ; \\
& K_{4}=-2 c K-z K_{2} ; \quad S_{1}=3 A_{2}-Q_{3}^{2} ; S_{2}=3 A_{4}-Q_{3}^{2} ; \quad S_{3}=3 A_{1}-Q_{5}^{2} ; \\
& S_{4}=3 A_{3}-Q_{5}^{2} ; S_{5}=3 A_{1}-Q_{1}^{2} ; S_{6}=3 A_{2}-Q_{1}^{2} ; S_{7}=3 A_{3}-Q_{2}^{2} ; \\
& S_{8}=3 A_{4}-Q_{2}^{2} ; S_{9}=3 A_{1}-Q_{4}^{2} ; S_{10}=3 A_{3}-Q_{4}^{2} ; S_{11}=p^{2}+Q_{4}^{2} ;
\end{aligned}
$$




$$
\begin{gathered}
G_{1}=-z-2 p K ; G_{2}=-G_{1} ; H_{1}=3 p^{2}+2 Q_{1}^{2}+3 Q_{3}^{2} ; \\
H_{2}=3 p^{2}+2 Q_{2}^{2}+3 Q_{3}^{2} ; H_{3}=3 p^{2}+2 Q_{1}^{2}+3 Q_{4}^{2} ; \\
H_{4}=3 p^{2}+2 Q_{2}^{2}+3 Q_{4}^{2} ; H_{5}=2 p^{2}+Q_{1}^{2}+Q_{3}^{2} ; \\
H_{6}=2 p^{2}+Q_{2}^{2}+Q_{3}^{2} ; H_{7}=2 p^{2}+Q_{1}^{2}+Q_{4}^{2} ; H_{8}=2 p^{2}+Q_{2}^{2}+Q_{4}^{2} ; \\
M_{1}=\frac{S_{10}}{B_{2}^{2}}+\frac{2 Q_{2}^{2}+3 S_{11}}{S_{11}^{2}} ; M_{2}=\frac{S_{1}}{B_{1}^{2}}+\frac{S_{6}}{C_{1}^{2}} ; M_{3}=\frac{S_{2}}{B_{2}^{2}}+\frac{S_{8}}{C_{1}^{2}} ; M_{4}=\frac{2 Q_{1}^{2}+3 S_{11}}{S_{11}^{2}}+\frac{S_{9}}{B_{1}^{2}} ; \\
M_{5}=\frac{6 p}{B_{2}^{2}}-\frac{4 p S_{10}}{B_{2}^{3}}+\frac{6 p}{S_{11}^{2}}-\frac{4 p\left(2 Q_{2}^{2}+3 S_{11}\right)}{S_{11}^{3}} ; M_{6}=\frac{6 p}{B_{1}^{2}}+\frac{6 p}{C_{1}^{2}}-\frac{4 p S_{1}}{B_{1}^{3}}-\frac{4 p S_{6}}{C_{1}^{3}} ; \\
M_{7}=\frac{6 p}{B_{2}^{2}}+\frac{6 p}{C_{1}^{2}}-\frac{4 p S_{2}}{B_{2}^{3}}-\frac{4 p S_{8}}{C_{1}^{3}} ; M_{8}=\frac{6 p}{B_{1}^{2}}+\frac{6 p}{S_{11}^{2}}-\frac{4 p\left(2 Q_{1}^{2}+3 S_{11}\right)}{S_{11}^{3}}-\frac{4 p S_{9}}{B_{1}^{3}} ; \\
M_{9}=\frac{Q_{1}}{\sqrt{A_{2}}}+\frac{Q_{2}}{\sqrt{A_{4}}} ; M_{10}=-\frac{Q_{1} Q_{3}}{A_{2}^{3 / 2}}-\frac{Q_{2} Q_{3}}{A_{4}^{3 / 2}} ; M_{11}=\frac{Q_{1}}{\sqrt{A_{1}}}+\frac{Q_{2}}{\sqrt{A_{3}}} ; \\
M_{12}=-\frac{Q_{1} Q_{4}}{A_{1}^{3 / 2}}-\frac{Q_{2} Q_{4}}{A_{3}^{3 / 2}} ;
\end{gathered}
$$

